\title{
The X-shooter GRB afterglow legacy sample (XS-GRB) ${ }^{\star}$
}

\author{
J. Selsing ${ }^{1,2,3}$, D. Malesani ${ }^{1,2,3,4, \star \star}$, P. Goldoni ${ }^{5,8, \star \star}$, J. P. U. Fynbo ${ }^{1,2,3, \star \star}$, T. Krühler ${ }^{6, \star \star}$, L. A. Antonelli ${ }^{7, \star \star}$, \\ M. Arabsalmani ${ }^{8,9}$, J. Bolmer ${ }^{6,10, \star \star}$, Z. Cano ${ }^{11, \star \star}$, L. Christensen ${ }^{1}$, S. Covino ${ }^{12, \star \star}$, P. D'Avanzo ${ }^{12, \star \star}$, \\ V. D’Eliaa ${ }^{13, \star \star}$, A. De $\mathrm{Cia}^{14}$, A. de Ugarte Postigo ${ }^{1,11, \star \star}$, H. Flores ${ }^{15, \star \star}$, M. Friiis ${ }^{16,17}$, A. Gomboc ${ }^{18}$, J. Greiner ${ }^{6}$, \\ P. Groot ${ }^{19}$, F. Hammer ${ }^{15}$, O. E. Hartoog ${ }^{20, \star \star}$, K. E. Heintz ${ }^{1,2,3,21, \star \star}$, J. Hjorth ${ }^{1, \star \star}$, P. Jakobsson ${ }^{21, \star \star}$, J. Japelj ${ }^{20, \star \star}$, \\ D. A. Kann ${ }^{11, \star \star}$, L. $\operatorname{Kaper}^{20}$, C. Ledoux ${ }^{10}$, G. Leloudas ${ }^{1}$, A. J. Levan ${ }^{22, \star \star}$, E. Maiorano ${ }^{23}$, A. Melandri ${ }^{12, \star \star}$, \\ B. Milvang-Jensen ${ }^{1,2,3}$, E. Palazzi ${ }^{23}$, J. T. Palmerio ${ }^{24, \star \star}$, D. A. Perley ${ }^{25, \star \star}$, E. Pian ${ }^{23}$, S. Piranomonte ${ }^{7, \star \star}$, \\ G. Pugliese ${ }^{20, \star \star}$, R. Sánchez-Ramírez ${ }^{26, \star \star}$, S. Savaglio ${ }^{27}$, P. Schady ${ }^{6}$, S. Schulze ${ }^{28, \star \star}$, J. Sollerman $^{29}$, \\ M. Sparre ${ }^{30, \star}$, G. Tagliaferri ${ }^{12}$, N. R. Tanvir ${ }^{31, \star \star}$, C. C. Thöne ${ }^{11}$, S. D. Vergani ${ }^{15, \star \star}$, P. Vreeswijk ${ }^{19,27, \star \star}$, \\ D. Watson ${ }^{1,2,3, \star \star}$, K. Wiersema ${ }^{22,31, \star \star}$, R. Wijers ${ }^{20}$, D. Xu ${ }^{32, \star \star}$, and T. Zafar ${ }^{33}$ \\ (Affiliations can be found after the references)
}

Received 15 February 2018 / Accepted 16 November 2018

\begin{abstract}
In this work we present spectra of all $\gamma$-ray burst (GRB) afterglows that have been promptly observed with the X-shooter spectrograph until 31/03/2017. In total, we have obtained spectroscopic observations of 103 individual GRBs observed within 48 hours of the GRB trigger. Redshifts have been measured for 97 per cent of these, covering a redshift range from 0.059 to 7.84 . Based on a set of observational selection criteria that minimise biases with regards to intrinsic properties of the GRBs, the follow-up effort has been focused on producing a homogeneously selected sample of 93 afterglow spectra for GRBs discovered by the Swift satellite. We here provide a public release of all the reduced spectra, including continuum estimates and telluric absorption corrections. For completeness, we also provide reductions for the 18 late-time observations of the underlying host galaxies. We provide an assessment of the degree of completeness with respect to the parent GRB population, in terms of the $\mathrm{X}$-ray properties of the bursts in the sample and find that the sample presented here is representative of the full Swift sample. We have constrained the fraction of dark bursts to be $<28$ per cent and confirm previous results that higher optical darkness is correlated with increased X-ray absorption. For the 42 bursts for which it is possible, we have provided a measurement of the neutral hydrogen column density, increasing the total number of published HI column density measurements by 33 per cent. This dataset provides a unique resource to study the ISM across cosmic time, from the local progenitor surroundings to the intervening Universe.
\end{abstract}

Key words. gamma-ray burst: general - galaxies: high-redshift - ISM: general - techniques: spectroscopic - catalogs - galaxies: star formation

\section{Introduction}

Gamma-ray bursts (GRBs) are bright glimpses of electromagnetic radiation that pierce through the Universe, all the way from the edges of the observable Universe. They provide constraints on a very wide range of topics in astrophysics. Examples range from small-scale phenomena relating to magnetars, properties of highly relativistic jets, hypernova and supernova explosions, the interstellar medium (ISM), dust extinction curves, starburst galaxies, chemical and molecular abundances, escape of ionising radiation, the ionization state of the intergalactic medium, intervening absorption systems to standard candles in cosmology (e.g. Wijers et al. 1998; Savaglio 2006; Ghirlanda 2007; Molinari et al. 2007; Amati et al. 2008; Vergani et al. 2009; Prochaska et al. 2009; Hjorth \& Bloom 2011; Rowlinson et al. 2017; Christensen et al. 2017).

The Neil Gehrels Swift Observatory (Swift) satellite (Gehrels et al. 2004, 2009), which was launched in 2004, has made it possible to harvest much of the great potential in using GRBs

* Based on observations collected at the European Southern Observatory, Paranal, Chile, Programme IDs: 084.A-0260, 084.D-0265, 085.A-0009， 086.A-0073， 087.A-0055，088.A-0051，089.A-0067, 090.A-0088, 091.A-0877, 0091.C-0934，092.A-0124，092.D-0056, 092.D-0633， 093.A-0069，094.A-0134，095.A-0045，095.B-0811, 096.A-0079, 097.A-0036, 098.A-0136, and 098.A-0055.

$\star \star$ On-call observer. as probes of the intergalactic medium, which has already been hinted at by results from earlier missions (e.g. van Paradijs et al. 2000; Ricker \& Team 2004). With the three on-board instruments, the Burst Alert Telescope (BAT; Barthelmy et al. 2005), the X-Ray Telescope (XRT; Burrows et al. 2005), and the UltraViolet and Optical Telescope (UVOT; Roming et al. 2005), Swift is an ideal observatory for GRB hunting. A crucial aspect of the success of the Swift mission has been the extensive ground-based follow-up observations of the afterglows and of the host galaxies of the GRBs, involving a large community of researchers. This fruitful collaboration has been facilitated by the open data access policy of the Swift mission. The close collaboration between detection facilities and electromagnetic follow-up campaigns continue to be immensely rewarding, as recently highlighted by the simultaneous detection of gravitational waves and light from the neutron star merger in the shape of GW170817/GRB170817A/AT2017gfo (Goldstein et al. 2017; Savchenko et al. 2017; Soares-Santos et al. 2017; Cowperthwaite et al. 2017; Nicholl et al. 2017; Chornock et al. 2017; Tanvir et al. 2017; Lipunov et al. 2017; Arcavi et al. 2017; Pian et al. 2017; Troja et al. 2017b; Smartt et al. 2017; Kilpatrick et al. 2017; Coulter et al. 2017; Burgess et al. 2017; Abbott et al. 2017; LIGO Scientific Collaboration et al. 2017).

In the beginning of the Swift era most of the followup afterglow spectroscopy was secured using low-resolution 
spectrographs (typically $R=\lambda / \Delta \lambda<1000$, e.g. Fynbo et al. 2009). Spectroscopy is powerful as it allows us to secure information even for very faint targets (Krühler et al. 2012a). This allows the measurement of a number of important parameters such as redshifts, spectral slopes, and extinction. For a handful of very bright afterglows high-resolution (typically $R>20000$ ) spectra have been secured, and for these events much more information about conditions inside the host galaxies were extracted (e.g. Fiore et al. 2005; Thöne et al. 2007; Prochaska et al. 2007; Vreeswijk et al. 2007; D'Elia et al. 2009; Castro-Tirado et al. 2010).

The X-shooter spectrograph (Vernet et al. 2011) was the first of the second generation instruments at the ESO Very Large Telescope (VLT). It was designed very much with transient follow-up in mind as the fading luminosities of such sources makes it urgent to secure as extensive wavelength coverage as possible in the shortest possible time. At the same time, the resolution was designed to be in the range 4000-9000 in order to be able to get a large useful spectral range between the many sky-background emission lines in the red and near-IR spectral ranges. The near-IR spectral coverage allows for spectroscopic observations of the highest redshift GRBs.

In this paper, we present the results of a dedicated effort over the years 2009-2017 to use the X-shooter spectrograph to secure spectroscopic observations of afterglows and host galaxies of GRBs detected by Swift. Here we make all the data resulting from the survey publicly available in reduced form (see Sect. 3.8).

The paper is organised in the following way: In Sect. 2 we describe the sample including the sample selection criteria and the observational strategy. In Sect. 2.4, we describe the observations and the instrumental setups, and in Sect. 3 we detail the methodological strategies adopted in the data reduction process and auxiliary material. In Sect. 4 we describe the results of the survey, the efficiency of the follow-up effort, and the characteristics of the observed bursts. We also assess the completeness of the realised sample. Finally, we offer our conclusions in Sect. 5 . We use the $\Lambda \mathrm{CDM}$ cosmology parameters provided by the Planck Collaboration XIII (2016) in which the Universe is flat with $H_{0}=67.7 \mathrm{~km} \mathrm{~s}^{-1} \mathrm{Mpc}^{-1}$ and $\Omega_{\mathrm{m}}=0.307$.

\section{Sample selection criteria and observations}

\subsection{Sample selection criteria}

Being of transient nature, it is difficult to impose strong sample selection criteria on GRBs without hampering the followup effort. Many natural follow-up restrictions exist already, being it weather conditions, pointing restrictions of the telescope or unconstrained burst localizations as reported by the alerting facility. To maximise the return of the follow-up campaign, we have chosen a few selection criteria that facilitate an unbiased selection of bursts, while at the same time allowing for a high follow-up success rate. The importance of defining unbiased selection criteria has been highlighted previously (Jakobsson et al. 2006; Salvaterra et al. 2012; Hjorth et al. 2012; Vergani et al. 2015; Perley et al. 2016a), when trying to address the intrinsic underlying distribution functions such as the redshift distribution, host metallicity distribution, or afterglow brightness distribution. When investigating a specific distribution function, a high degree of completeness is desired (e.g. Perley et al.2016b).

In defining the selection criteria, we simultaneously aim to minimise any biases against intrinsic astrophysical conditions while at the same time maximising the likelihood of successful observations, hence allowing us to obtain a higher degree of completeness. By restricting the selection criteria to conditions local to the Milky Way and therefore independent of intrinsic GRB properties, the aim is that the collected sample represents the underlying distribution of GRBs in a fair way. The selection criteria used here are based on previous, similar studies (Jakobsson et al. 2006; Fynbo et al. 2009; Hjorth et al. 2012). We characterise the sample completeness in Sect. 4.2.

The selection criteria that define a GRB as part of our initial statistical sample are:

1. GRB trigger by BAT on-board the Swift satellite

2. XRT started observing within ten minutes after the GRB; an XRT position must be distributed within $12 \mathrm{~h}$.

3. The target must be visible from Cerro Paranal for at least $60 \mathrm{~min}, 30^{\circ}$ above the horizon, with the Sun below $-12^{\circ 1}$.

4. Galactic $A_{V} \leq 0.5 \mathrm{mag}$ according to the maps of Schlegel et al. (1998).

5. No bright, nearby stars within $1.8+0.4 \times \exp [(20-R) / 2.05]$ $\operatorname{arcsec}$, where $R$ is the USNO magnitude of the star.

Our ability to observe GRB afterglows is strongly dependent on the timing and the precision of the target positions delivered by the triggering facilities. By selecting only bursts that have been triggered on board the Swift space telescope (Gehrels et al. 2004), based on the BAT, we start out with a sample where burst characteristics are delivered immediately, allowing for an informed follow-up strategy. However, the BAT sensitivity varies across its field of view, so selection is not entirely homogeneous. Despite the complexity of the triggering mechanism on board Swift (Band 2006; Coward et al. 2013), attempts at inferring properties of the underlying GRB population based on the detection thresholds and triggering algorithms have been made (Lien et al. 2014; Graff et al. 2016). Restricting the follow-up effort to bursts detected by Swift, we therefore ensure that the limitations of the parent sample are well studied.

Because the localization accuracy of BAT is 1-4 arcmin (Barthelmy et al. 2005), an afterglow identification based on BAT alone would be harder, and host association impossible. We therefore additionally require an X-ray position from XRT to be distributed to the GCN network (Barthelmy 2000) within $12 \mathrm{~h}$ and to account for observing constraints on Swift that XRT began observations within ten minutes. The additional timing requirement of the XRT follow-up means that all bursts in our sample have detected X-ray afterglows. Because the XRT completeness is very high for promptly-repointed GRBs (Burrows et al. 2007), this cut should not alter the parent sample significantly.

To ensure a minimum of observability, we require that the GRB is visible from the telescope site at Cerro Paranal, Chile, for a least one hour after the trigger with the sun below $-12^{\circ}$. This secures time for the spectroscopic observations to be completed. Since the GRB population is isotropically distributed on the sky as seen from Earth, and because the GRB properties do not depend on position on the sky (Meegan et al. 1992; Briggs et al. 1996; Ukwatta \& Wózniak 2016), this cut does not influence our ability to fairly sample the underlying GRB population. The same arguments apply to the requirement that there are no nearby foreground bright stars. We additionally require that the Galactic extinction is below $A_{V} \lesssim 0.5 \mathrm{mag}$, based on the

\footnotetext{
1 We note that in the P84 proposal the criteria have been stated differently, the visibility constraint being replaced by a declination + Sun angle constraint. The criteria listed above are, however, those defining the sample.
} 
extinction maps by Schlegel et al. $(1998)^{2}$. Choosing lowextinction sightlines also reduces the problem of field crowding and the contamination from Galactic outbursts posing as GRB impostors. These additional cuts should not influence the optical properties of the bursts themselves, only our ability to successfully secure the observations that allow us to investigate the spectroscopic properties of GRBs.

Bursts that fulfill these selection criteria are what we define as our initial statistical sample. To reach a higher degree of sample completeness, we impose additional cuts that increase our ability to infer population properties. These are presented in Sect. 4.2. Using this sample we will be able to address population properties of Swift-detected bursts. We further discuss the effect of these selection criteria and their implication for the completeness of the sample in Sect. 4.2.

We remark, however, that while the selection of the afterglows has been carried out in a homogeneous way, the resulting quality of the spectra is not uniform. Individual bursts vary significantly in brightness, due to intrinsic differences in luminosity, dust extinction, time of observation, etc. The covered rest-frame wavelength range is also different for each case due to the different redshifts of each GRB. Given that our observations are targets-of-opportunity, this leads to a varying $\mathrm{S} / \mathrm{N}$, and in particular the sensitivity limit to detect absorption features varies greatly from burst to burst and as a function of wavelength.

\subsection{Follow-up procedure}

Our collaboration has set up a procedure to promptly react to alerts from Swift and other satellites (such as Fermi). We process automatically GCN notices in order to flag those events belonging to our statistical sample. Two people are permanently on alert to manually supervise each event, for example to recognise events of special interest beyond their inclusion in the sample. Members of our collaboration have access to numerous facilities spread throughout the world, among which the William Herschel Telescope (WHT), Nordic Optical Telescope (NOT), the Gran Telescopio Canarias (GTC), the Telescopio Nazionale Galileo (TNG), the Gamma-Ray Burst Optical Near-infrared Detector (GROND; Greiner et al. 2008), and the Xinlong Observatory. Thanks to this network, we can often conduct searches for optical/NIR afterglows ahead of the time of the X-shooter spectroscopy, thus helping to plan the spectroscopic observations. If this is not possible, we use the built-in acquisition camera of $\mathrm{X}$-shooter to search for counterparts, and adapt the observing strategy on the fly. In many cases we could interact directly with the staff at the telescope to aid the observation. Raw data are usually available within minutes in the ESO archive $^{3}$ and are promptly reduced by members of our collaboration, often using archival calibration data which are readily available. This allows us in most cases to report the preliminary results (redshift, identification of the most prominent emission and absorption feature, etc.) within a few hours after the beginning of the observations and plan additional X-shooter observations if deemed necessary.

\subsection{Rapid response mode (RRM) observations}

Under rare circumstances, the use of the ESO rapid response mode (RRM; Vreeswijk et al. 2010) has been possible. The

\footnotetext{
2 We use the updated values of Schlafly \& Finkbeiner (2011) to correct for foreground extinction, but the sample criterion is based on the old Schlegel et al. (1998) values for consistency with the first semesters of our programme.

3 http://archive. eso.org/wdb/wdb/eso/xshooter/form
}

RRM is a system to automatically override ongoing observations at the telescope. This allows the shortest possible delay between the GRB trigger and the initiation of observations, where spectroscopic integration of the rapidly fading, optical transient can commence within minutes of the burst. One limitation is that no instrument change is allowed in RRM, which lowers the number of successful triggers (X-shooter shares the telescope with two other instruments). In case of a promptly visible GRB, a robotic trigger is sent to the telescope if at the time of the GCN notice the GRB fulfills the following criteria:

1. The GRB triggered Swift.

2. The X-ray position must be available less than one hour after the GRB.

3. The elevation of the source in the sky is $>22^{\circ}$ (both at trigger time and $15 \mathrm{~min}$ after).

4. The Sun elevation from Paranal is $<-12^{\circ}$ (both at trigger time and $15 \mathrm{~min}$ after).

5. Galactic $A_{V} \leq 1.0 \mathrm{mag}$ according to the maps of Schlegel et al. (1998).

6. The Swift-circulated tags: KNOWN_SOURCE, COSMIC_RAY, DEF_NOT_GRB are set to false (PROB_NOT_GRB can be true $)^{4}$.

The criteria to trigger RRM are looser than those that define the statistical sample. This is both because of the expected larger brightness of GRB counterparts soon after the explosion, and because of the rarity of RRM triggers.

The use of RRM is unique as it allows to sample a long logarithmic time span in the GRB lifetime, and it exploits the extreme brightness of early afterglows. A pivotal example is the study of temporal variability of GRB afterglow absorption systems due to effect of the GRB itself on the surrounding medium (e.g. see Dessauges-Zavadsky et al. 2006; Vreeswijk et al. 2007, 2013; D'Elia et al. 2009).

There are nine GRBs that have been observed with X-shooter in RRM mode. One of the RRM triggers is outside the statistical sample, and two of the RRM triggers are on short GRBs. Our fastest response (between the Swift GRB trigger time and the beginning of spectroscopic integration) was for GRB 160410A, for which the delay was only $8.4 \mathrm{~min}$.

In many cases, unfortunately, we could not use RRM even for promptly visible events, e.g. because of the unavailability of X-shooter. Potentially, the proximity of the telescope (Cerro Paranal, Chile) to the South Atlantic Anomaly (SAA) affects the rate of RRM triggers (Greiner et al. 2011). BAT switches off when the satellite goes through the SSA and that means we have fewer events immediately observable, compared to the rest of the Swift orbit.

\subsection{Observations}

The observations obtained for this sample have been secured with the cross-dispersed echelle spectrograph, X-shooter (Vernet et al. 2011), mounted on one of two Unit Telescopes at ESO/VLT, UT2 (Kueyen) and UT3 (Melipal) during the duration of this follow-up campaign. The observations have been taken during a period of eight years corresponding to the ESO observing periods P84 through P98 under the following programme IDs: 084.A-0260, 085.A-0009, 086.A-0073, 087.A-0055, 088.A-0051, 089.A-0067, 090.A-0088, 092.A0124, 093.A-0069, 094.A-0134, 095.A-0045, 096.A-0079,

\footnotetext{
4 These tags are distributed as part of the GCN notices. Explanations for all tags are at https://gcn.gsfc.nasa.gov/sock_pkt_def_ doc. html
} 
097.A-0036, and 098.A-0055 (PI: Fynbo) and 0091.C-0934 (PI: Kaper). These proposals were initiated on Guaranteed Time. We have included a few additional bursts, from the programmes 084.D-0265 (PI: Benetti), 091.A-0877 (PI: Schady), 092.D-0056 (PI: Rau), 092.D-0633, 098.A-0136 (PI: Greiner), and 095.B0811 (PI: Levan). The total collection of spectra represents all GRB afterglows that have been followed up by X-shooter up to $31 / 03 / 2017$, which marks the end of the XS-GRB legacy follow-up programme.

The first GRB followed up was GRB 090313 (de Ugarte Postigo et al. 2010), observed on the 15th of March, 2009, during the commissioning of X-shooter on the second Unit Telescope (UT2) of the VLT . The bursts observed during the commissioning or the science verification process (GRB 090313, GRB 090530, GRB 090809, GRB 090926A) are not a part of the sample we use to investigate the statistical properties of GRB afterglows, due to the different criteria for their selection. The first burst observed after science verification and the mounting of X-shooter on UT2, was GRB 091018, which is the first burst entering our homogeneously selected statistical sample. For all bursts that fulfill our sample selection criteria, described in Sect. 2.1, spectroscopic follow-up have been attempted with X-shooter. Various conditions can affect our ability to follow up a given burst, and a discussion of these conditions and their consequences for the sample is included in Sect. 4.2.

$\mathrm{X}$-shooter covers the spectral wavelength region from $300 \mathrm{~nm}$ to $2480 \mathrm{~nm}$ in a single exposure, by splitting the light into three separate spectroscopic arms through the use of two dichroics. The ultraviolet blue (UVB) arm covers 300-550 nm, the visual (VIS) arm covers $550-1020 \mathrm{~nm}$, and the near-infrared (NIR) arm covers 1020-2480 nm. For some of the observations, we have applied a $K$-band blocking filter, cutting the coverage of the NIR arm at $2100 \mathrm{~nm}$. The $K$-band blocking filter is only used after 2012, where it was installed. This is done to reduce the amount of scattered background light from the thermal infrared. For the majority of observations, a nodding observing scheme has been employed, with a nodding throw of $5^{\prime \prime}$. Each nodding observation has typically been carried out in a standard ABBA pattern. For some cases, conditions during the followup (either technical or weather), have necessitated alterations to this scheme as described in Appendix C. For RRM triggers, a slightly different observing strategy was employed. Starting as rapidly as possible, a simple stare mode sequence was started, with FIVE spectroscopic integrations with increasing exposure times.

For the majority of the bursts, we have observed with a slit width of $11^{\prime \prime} 0,0.9$, and 0.9 for the UVB, VIS, and NIR-arm respectively. This sets a lower limit on the delivered resolving power of the spectra based on the tabulated values of the delivered resolutions, which is 4350, 7450, and 5300 for the UVB, VIS and NIR-arm respectively ${ }^{5}$. For accurate measurements involving line profiles, knowledge of the precise instrumental resolution is required. The spectral resolution becomes better than the nominal one, when the delivered seeing is smaller than the projected width of the slit on the sky. We discuss how we determine the effective instrumental resolution in Sect. 3.3.

Due to a mechanical failure, the atmospheric dispersion corrector (ADC) was disabled from 1st of August 2012 until the end of this programme. Only GRB 100728B was affected by the fail-

\footnotetext{
5 See https://www.eso.org/sci/facilities/paranal/ instruments/xshooter/inst.html for the nominal spectral resolutions.
}

ing ADC prior to disablement, resulting in a lower-than-nominal throughput. To avoid chromatic slit-loss due to atmospheric dispersion, nearly all subsequent observations have been carried out at parallactic angle. A consequence of this is that for all observations following 1st of August 2012, the centroid of the trace of the source changes position across the spatial direction of the slit as a function of wavelength. This effect has been modelled in the extraction procedure, as described in Sect. 3.4.

We provide an overview of all the observations in Table A.1 and plot the positions of all the bursts on the celestial sphere in Galactic coordinates in Fig. 1. Away from the central zone of avoidance, due to high Galactic extinction cutoff (marked in white), the GRB positions have an isotropic distribution, except in the upper left quadrant which cannot be observed from Paranal due to the declination constraints of the telescope.

Thirty percent of the spectra presented here, primarily host observations, have already been published in Krühler et al. (2015a). Single bursts have additionally been published, based on unusual properties in their afterglows. We present individual notes on all the X-shooter spectra and their previous use in Appendix C. We include independent reductions of them here for completeness.

\section{Data processing}

In this section we describe how the final data products are produced and subsequently post-processed. All post-processing scripts developed for this dataset are made publicly available online ${ }^{6}$.

Before any reductions are initiated, the raw object images are run through the cosmic-ray removal algorithm (van Dokkum 2001) implementation, Astro-SCRAPPY ${ }^{7}$, where a wide clipping radius was used around detected cosmic ray hits to ensure that edge residuals are robustly rejected.

The basis for the reductions is the VLT/X-shooter pipeline, version 2.7.1 or newer (Goldoni et al. 2006; Modigliani et al. 2010). The pipeline is managed with the Reflex interface (Freudling et al. 2013) and is used for subtraction of bias level, flat-fielding, tracing of the echelle orders, wavelength calibrations with the use of arc-line lamps, flux calibration using spectrophotometric standards (Vernet et al. 2009; Hamuy et al. 1994), mirror flexure compensation(see Sect. 3.2), sky-subtraction and lastly the rectification and merging of the orders. Errors and bad pixel maps are propagated throughout the extraction. For the initial sky-subtraction, the background has been estimated by a running median in regions adjacent to the object trace clear of contaminating sources. Due to the broken ADC, for some objects there is curvature in the object trace along the dispersion axis of the slit (see Sect. 3.4). This means that for these bursts, the initial sky-estimate was made from a limited number of pixels in the spatial direction. The subtraction of the sky background on the un-rectified image ensures that the bulk of the sky background is not redistributed by the rectification process.

$\mathrm{X}$-shooter is an echelle spectrograph, and therefore the individual echelle orders are curved across image space. The individual orders therefore need to be rectified. In order to transform the image space (pixels) into a physical (wavelength-slit) space, the image pixels are resampled onto a physical grid, while propagating the pixel uncertainties derived by the pipeline. This rectification process correlates neighboring pixels and in order

\footnotetext{
6 https://github.com/jselsing/XSGRB_reduction_scripts https://github.com/astropy/astroscrappy
} 


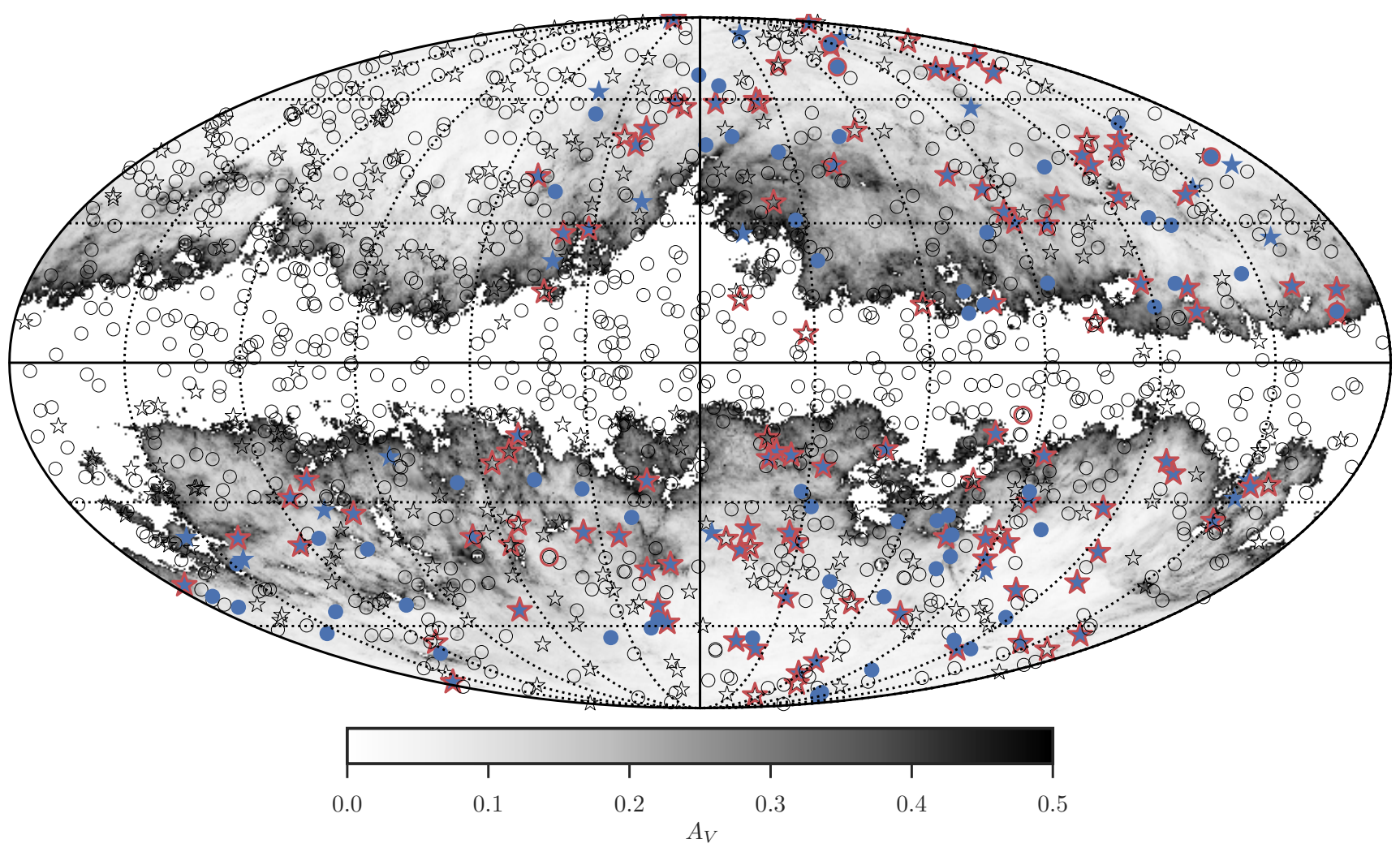

Fig. 1. Mollweide projection in Galactic coordinates of the full sky showing the positions on the sky of the bursts presented in this work. The equator is the Galactic plane. The empty stars/circles are the positions of all the 1266 Swift bursts detected until 31/03/2017. Stars indicate bursts with measured redshifts and circles indicate those without. Blue stars indicate the position of the 104 bursts fulfilling the sample criteria specified in Sect. 2.1 that have a measured redshift. Red outlines are added to the 84 GRBs that enter our statistical sample with both X-shooter spectroscopy and a measured redshift. Red outlines of empty stars represent bursts which have been observed with X-shooter and has a measured redshift, but is not a part of our statistical sample. The blue dots show the positions of the 61 GRBs of our statistical sample that lack redshift measurements. A red outline is added around the six bursts in our statistical sample which were observed with X-shooter, but did not yield a redshift measurement. Two empty circles with red outlines indicate bursts outside the statistical sample that were followed up with X-shooter, but without a redshift measurement. The different samples are compared in Sect. 4. The background shows the dust maps presented in Schlegel et al. (1998). We note that we removed the background where the sample criterion is violated $\left(A_{V}>0.5 \mathrm{mag}\right)$ and replaced it with a white background. The grey scale bar below indicates the value of $A_{V}$ on the plot. The dotted lines indicate intervals of $30^{\circ}$ in longitude and latitude.

to minimise the degree of correlation, we need to choose a physical sampling that matches the pixel sampling. We rectified the image onto an equidistant grid with a dispersion sampling of $0.02 \mathrm{~nm} \mathrm{pixel}^{-1}$ and a 0 "' 16 per pixel spatial sampling for the UVB and VIS arm and $0.06 \mathrm{~nm}_{\text {pixel }}{ }^{-1}$ with a $00^{\prime \prime 2}$ per pixel in the NIR arm. Because the tabulated resolution is a lower limit to the delivered resolution, we choose a sampling of $0.02 \mathrm{~nm} \mathrm{pixel}^{-1}$ to ensure that the lowest wavelength part of neither of the arms have a sampling lower than the Nyquist sampling rate of two pixels per resolution FWHM.

\subsection{Post-processing}

For a typical observation, each of the exposures in the nodding sequence have been reduced as a single observation and then subsequently combined to form a single image. We employ this strategy so that we can reject outliers in the stack and weight by an averaged measure of the inverse variance of the background. When weighting images, where the noise in each pixel is dominated by Poisson noise, it is important to estimate the background variance in a large enough region, so that any correlation between the signal and the weights are removed. To this end, the weight map is generated by a running median window over the variance map produced by the pipeline, where the trace has been masked and the width of the window is chosen to be wide enough for the median variance to be calculated on the basis of several hundred pixels. This weighting scheme automatically also optimally combines images of different exposure times or images where the background is varying, which is often the case when a burst has been observed close to twilight.

An additional sky-subtraction procedure is run on all rectified 2D spectra. This is done to remove residual sky, still present. At each pixel in the dispersion direction, the spatial dimension is fit with a low-order polynomial, after all sources are masked out. This low order polynomial is then convolved with a few pixel wide Gaussian filter in the dispersion direction and subtracted from the entire $2 \mathrm{D}$ image.

In the NIR arm, where the background is very bright and there are a high number of bright sky-lines, an alternative approach to sky subtraction has been employed. When there are no contaminating sources in the slit, the sky has been put back on the images and the images are combined in pairs of two before extraction, subtracting the two from each other while keeping the WCS static. Due to the nodding offsets used between observations, this conserves the source flux while removing the sky at the expense of a decrease in signal-to-noise $(\mathrm{S} / \mathrm{N})$ by a factor of $\sqrt{2}$. This amounts to the regular nodding reduction, only we can reject outliers and weight by the averaged inverse variance map. 
Reducing the images as single observations for all exposures, we additionally get a spectrum of the sky which we can use to recalibrate the wavelength solution in the post-processing steps.

\subsection{Correction for offsets in the wavelength calibration}

Since X-shooter is installed at the VLT Cassegrain focus, it is prone to flexures. The flexures modify the projection of the slit on the detector with respect to the one obtained in daytime calibration. This requires a modification of the wavelength solution in order to correctly process the night-time data. Part of this correction is performed by the pipeline using the frames taken during the X-shooter Active Flexure Compensation procedure ${ }^{8}$.

The remaining offset is corrected by cross-correlating the observed sky spectrum with a synthetic sky spectrum (Noll et al. 2012; Jones et al. 2013) after the continuum, estimated as the mode of all flux values, has been subtracted. To get the correct seeing point-spread-function (PSF) with which to convolve the synthetic sky, an initial refinement of the wavelength solution has been obtained by cross-correlating the observed sky with an unconvolved synthetic sky. This preliminary wavelength calibration is applied to the observed sky. The synthetic spectrum is then convolved with an increasing seeing PSF and the width that minimises $\chi^{2}$ with the updated observed sky is chosen to be the effective sky-PSF. Using the synthetic sky with the matched resolution, a final wavelength calibration can then be calculated by cross-correlating the observed sky with the correctly broadened sky spectrum as a function of a velocity offset. Both a multiplicative and an additive offset to the wavelength calibration has been tested, but in terms of $\chi^{2}$, the model with only a multiplicative offset is preferred. The resulting offsets, which were smaller than $0.01 \mathrm{~nm}$ in the UVB and VIS data and smaller than $0.05 \mathrm{~nm}$ in the NIR spectra, but changing over short periods of time were applied to the corresponding spectra ${ }^{9}$. Using the convolved synthetic sky, the pixels containing the brightest sky lines have been flagged as such in the bad pixel map.

The resulting offsets are generally small, of the order of $\sim 5 \mathrm{~km} \mathrm{~s}^{-1}$, and thus do not influence significantly the redshift measurements, whose uncertainties are primarily driven by the $\mathrm{S} / \mathrm{N}$ of the spectra, the line-shapes, and the choice of ion species. Such a correction, however, can improve the accuracy of line fits, especially when multiple components are tied together, across a wide wavelength range, and allows a better comparison with high-resolution data. The modest size of the correction additionally serves to confirm the accuracy of the wavelength solution.

\subsection{Spectral resolution}

The afterglow spectra described in this paper are obtained in Target-of-Opportunity mode. In most cases, there is little possibility to tweak slit widths to the seeing at the time of observations (for instance to optimise spectral resolution and $\mathrm{S} / \mathrm{N}$ ), and almost all our data are therefore taken with a fixed set of slit widths and binning, described above. In a fair number of cases, the seeing full width at half maximum (FWHM) is considerably smaller than the slit width, and the delivered spectral resolution will then be determined by the seeing rather than by the slit

\footnotetext{
8 X-shooter User Manual available at ESO website: https: //www . eso.org/sci/facilities/paranal/instruments/ xshooter/doc.html

9 The wavelength shifts have been extensively studied by ESO staff https://www. eso.org/sci/facilities/paranal/instruments/ xshooter/doc/XS_wlc_shift_150615.pdf
}

width, as afterglows are point sources (this is evidently not the case for extended sources, e.g for host galaxies). The delivered resolution for slit-width dominated spectra post-reduction and extraction can easily be determined from the bright sky emission lines. For afterglow spectra with very high $\mathrm{S} / \mathrm{N}$, the delivered spectral resolution can at times be determined from the science data themselves. However, in the presence of multiple velocity components in absorption, other forms of line broadening, and a lack of lines at some redshifts, this is difficult to do at lower $\mathrm{S} / \mathrm{Ns}$ (the majority of spectra in our sample). A broad starting value for the expected resolution will help fitting of these spectra, and can be important in upper limit determination, and for this reason we construct a crude relation between the seeing and the delivered resolution at our slit width, binning, and reduction pipeline settings.

To this end we use observations of telluric standard stars that are taken with identical instrument settings as our afterglow spectra, usually just after the science data, as part of the ESO $\mathrm{X}$-shooter calibration plan. These spectra have been reduced together with the afterglow spectra, using identical pipeline settings with the same version of the pipeline. To get the resolution for each observation, we select a series of atmospheric transitions that are resolved multiples which should be intrinsically unresolved, and are in areas with well defined continuum flux. We then fit the lines with Voigt-profiles and calculate a corresponding FWHM, which we can then convert to a delivered resolution using the wavelength of the chosen transitions. To get the resolution as a function of ambient conditions, for each observations we also calculate the airmass-corrected DIMM ${ }^{10}$ seeing, which is measured at $500 \mathrm{~nm}$.

The resulting distribution of spectral FWHM $\left(\mathrm{km} \mathrm{s}^{-1}\right)$ as a function of spatial FWHM at $500 \mathrm{~nm}$ is fairly well described in the VIS arm by a linear relation $a+b * x$, with $x$ the spatial FWHM in arcsec, $a=25.2 \pm 0.8 \mathrm{~km} \mathrm{~s}^{-1}, b=3.8 \pm 0.7$ (see Fig. 2). For the NIR arm the corresponding relation is $a=36.0 \pm 1.8 \mathrm{~km} \mathrm{~s}^{-1}$, $b=8.4 \pm 1.8$. We use these linear relations as a way to estimate the spectral resolution for medium to low $\mathrm{S} / \mathrm{N}$ afterglow spectra. To extend this to the UVB arm, we calculate the ratio between the VIS and the NIR arm resolutions and find that the resulting distribution is consistent with a simple scaling of the VIS arm relation by the ratio of resolutions of the NIR and VIS arm for unresolved, slit filling, sources as given on the ESO instrument website $^{11}$. The UVB arm contains no suitable absorption lines to use, and we therefore use a scaled value. This simple analysis gives a sufficiently accurate estimate for the analysis of the low $\mathrm{S} / \mathrm{N}$ science spectra. In all cases the determined resolution is written to the header with the "RESOLUTION" keyword.

\subsection{Spectral extraction}

To extract the afterglow spectrum from the rectified 2D-image, several techniques have been employed based on the brightness of the afterglow and the complexity of the objects entering the slit. Due to the malfunctioning ADC (see Sect. 2.4), the spectral trace changes position across the slit in the spatial direction as a function of wavelength. For a large fraction of the observed bursts, using a single aperture for the spectral extraction is inadequate due to the large amount of background that would then enter the slit. To optimally select the extraction regions we therefore need to model the trace position.

\footnotetext{
10 http: //www . eso.org/asm/ui/publicLog

11 https://www . eso.org/sci/facilities/paranal/ instruments/xshooter/inst.html
} 


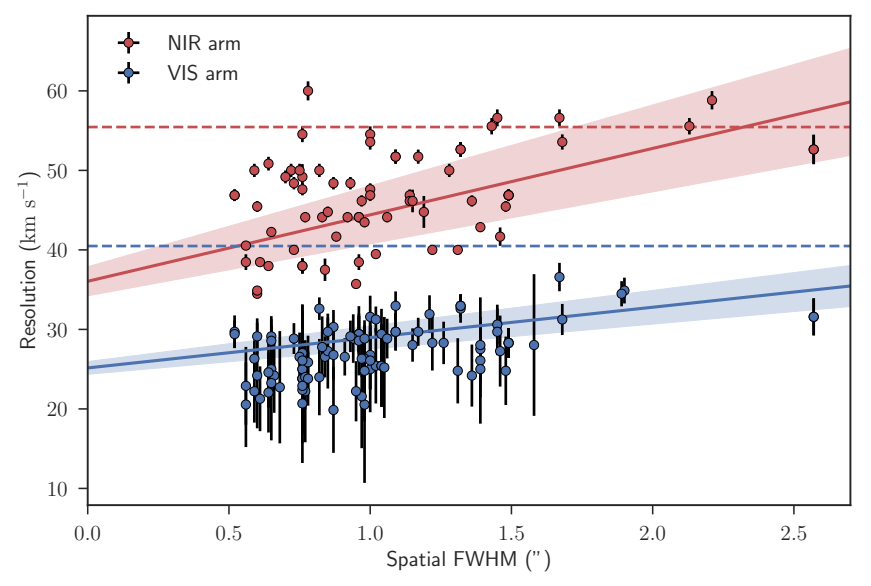

Fig. 2. Blue data points show the FWHM $\left(\mathrm{km} \mathrm{s}^{-1}\right)$ of Gaussian fits to unresolved telluric absorption lines in the VIS spectra, as a function of the airmass corrected DIMM seeing. The red data points show a subsample of measurements obtained for NIR spectra. The coloured lines show the corresponding linear fits to the data points. The coloured bounds contain 68 per cent of the best-fit probability mass. The red and blue dashed lines indicate the nominal resolution for the slit-widths used for the telluric standard observations. As can be seen, the effective resolution is in many cases superior to the nominal one.

To get the shape and the position of the spectral PSF as a function of location on the image, we need to chose a model which can represent how the light falls on the slit. We know from Trujillo et al. (2001) that a Moffat function (Moffat 1969) with an index of $\beta=4.765$ adequately describes an imaging PSF due to atmospheric turbulence, but because of aberrations in the optical dispersion elements and the rectification process, the PSF we are trying to model is different from this profile. To allow for flexibility in the model, we have chosen the Voigt function as a model for the spectral PSF and we describe how this is evaluated in Appendix B. Since the host galaxy could also give a contribution to the image profile, this choice allows for the required freedom if additional flux is in the wings of the profile.

To guide the estimated position of the trace on the slit as a function of wavelength, we have used the analytic prescription for the trace position described in Filippenko (1982), where the header keywords of the observations have been queried for the ambient conditions which controls the degree to which the trace changes position in the spatial direction. This analytic approach is only valid for a plane-parallel atmosphere, but because the final position is refined in the fit, it is adequate for our purposes.

Based on the $\mathrm{S} / \mathrm{N}$ of the afterglow continuum, the 2D-image has been binned in the spectral direction to a number of elements that allows for an accurate tracing of the PSF, typically 200 bins for moderate $\mathrm{S} / \mathrm{N}$. For each of the bins, using the analytically guided guess position, the spectral PSF has been fit using the unweighted chi-squared minimisation algorithm implemented in scipy .optimize.curve_fit (Oliphant 2007). Since we know that the trace varies slowly as a function of wavelength, we have then fitted a low-order polynomial to the fit parameters as a function of wavelength, which allows us to evaluate the spectral PSF at all wavelengths and in this way accurately model the entire spectral PSF.

Equipped with a model for how the light is distributed across the entire dispersion direction, we can employ the optimal extraction algorithm (Horne 1986), which weights the extraction aperture by the spectral profile, or alternatively sum all pixels within 1 FWHM of the modelled profile. Where possible, we

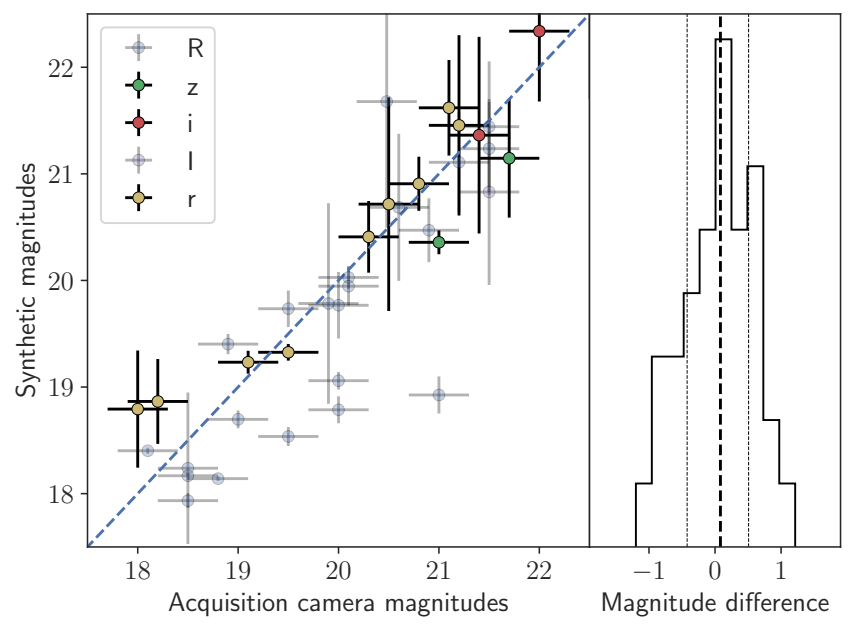

Fig. 3. Left panel: acquisition camera magnitudes as a function of synthetic photometry derived from the spectra. The blue, dashed line represent equality between the two measures. The colouring of the datapoints shows the photometric passband used by the acquisition camera, as indicated by the labels. The $R$ - and $I$-band magnitudes have been calibrated against the USNO-B1 catalogue, which has a poor photometric accuracy; this contributes significantly to the observed scatter, and for this reason we indicate these data with a light colour. Right panel: histogram of the differences between the two. The vertical lines in the histogram indicates the 16th, 50th, and 84th percentiles of the distribution $\left(\Delta \mathrm{mag}=0.07_{-0.45}^{+0.51}\right)$. This shows the accuracy of the flux calibration.

have used the optimal extraction. In cases where the trace is very weak, even in the binned images, an aperture has been selected manually which covers all emission lines, if present, and when nothing is immediately visible, the entire nodding window. The error- and bad pixel maps are in all cases propagated throughout the extraction.

In cases where multiple traces are visible in the slit, additional components for the profile are used in the optimal extraction. The additional components do not share the PSF parameters and in cases where the additional component is an extended object, the fits have been inspected to ensure that the additional component does not skew the fit towards a different PSF. The additional components are not used for the weights in the extractions.

The spectra are corrected for Galactic extinction using the $E(B-V)$ value from the dust maps of Schlegel et al. (1998) with the update in Schlafly \& Finkbeiner $(2011)^{12}$, and the extinction curve by Cardelli et al. (1989) with a total to selective extinction $R_{V}=3.1$. The wavelengths of the extracted 1D-spectra are wavelength recalibrated (described in Sect. 3.2), moved to vacuum, and corrected for barycentric motion. Pixels with pixel-topixel variation larger than $50 \sigma$ are additionally added to the bad pixel map.

\subsection{Slit-loss correction and flux calibration accuracy}

For the majority of the observations presented in this sample, the slit width of the target observations is less than the seeing PSF. This means that a fraction of the incoming light is lost outside the slit. This slit-loss is not corrected by the flux calibration, as flux standard stars are always observed with a 5"slit. As a consequence, almost all spectra will, without slit-loss correction,

12 Queried from http://irsa.ipac.caltech.edu/applications/ DUST/index.html using astroquery (Ginsburg et al. 2016). 
underestimate the flux by some fraction. Additionally, slit-loss is wavelength dependent due to the wavelength dependence of seeing (Boyd 1978) and as the seeing changes across the slit in wavelength, so does the slit-loss. This artificially introduces a change in continuum slope.

To correct for the slit-loss, we developed an algorithm that can, based on the atmospheric conditions at the time of observation and the wavelength dependence of seeing, estimate the losses at each wavelength bin. We start with the DIMM seeing (Sarazin \& Roddier 1990), which measures the seeing FWHM at zenith. We correct the zenith seeing for the median airmass of the observational sightline, which gives us the effective seeing, delivered at $5000 \AA$. We then use the fact that the seeing scales as $\sim \lambda^{-1 / 5}$ to obtain a measure of the seeing across the entire wavelength coverage of X-shooter. At each wavelength bin of the spectra, we simulate a synthetic 2D seeing PSF based on a normalised, 2D Moffat profile which we integrate over the used slit width. This can be used to correct for the estimated slit-loss at each wavelength bin.

To assess the accuracy of the flux-calibration, after the slitloss correction, we compare a subset of afterglow spectra with photometry derived from the acquisition camera. The acquisition camera takes a short image just before the spectroscopic integration, which only introduces a minimal delay and allows us to secure photometry for the vast majority of events. As photometric flux standards are rarely acquired, we resort to sky surveys such as the USNO catalogue (for the $R$ and $I$ bands; Monet et al. 2003), SDSS, or Pan-STARRS (griz; Abolfathi et al. 2018; Chambers et al. 2016), where available. The acquisition camera is equipped with similar filters, so we can avoid transformation between filter curves when comparing catalogues.

To compare the photometry with the spectral flux calibration, we calculate synthetic magnitudes from the spectrum, in the used passbands, along with the associated error. Using this set we can make a test of the final flux-calibration accuracy, which we show in Fig. 3. As can be seen, there is a general agreement between the flux-calibration and the derived acquisition magnitudes, although with significant scatter. The right panel in Fig. 3 shows a histogram of the differences, which can be summarised with $\Delta$ mag $=0.07_{-0.45}^{+0.51}$, where the errors are the 16th and 84th percentiles of the distribution. The scatter is smaller if we exclude measurements calibrated against the USNO catalogue, which is known to have a poor accuracy, also because, given the small field-of-view, we often need to rely on faint reference stars.

Similar to what is found in López et al. (2016), there is in some cases a mismatch in the flux-calibration between the spectroscopic arms. It is outside the scope of this work to correct for these offsets on an individual burst basis. The flux calibration of the spectra presented here shows a general good agreement with photometry although with some scatter. The spectral shape within the individual arms is correct, but the absolute scaling might in some cases be discrepant.

\subsection{Telluric correction}

For all Earth-based telescopes, the light first has to pass through Earth's atmosphere, where the atmospheric content and conditions make an imprint on the received spectrum. These telluric features can be corrected for in a multitude of ways. We employ a prioritised list of methods here, depending on the availability of the chosen methods. Since the observations are often taken at odd times under varying conditions, this prioritised list ensures that we are always doing the best possible correction.
The highest priority method is using the GRB afterglow continuum itself, where the atmospheric conditions have directly been imprinted on the spectrum. The telluric features can directly be fit with an atmospheric model (Molecfit; Smette et al. 2015; Kausch et al. 2015) ${ }^{13}$, which can then be used to correct for the absorption. The accuracy of the correction depends on the $\mathrm{S} / \mathrm{N}$ per pixel of the target spectrum, where we have chosen the requirement that the afterglow continuum spectrum has a median $\mathrm{S} / \mathrm{N}$ higher than a value of 10 .

If the afterglow is not sufficiently bright, telluric standard stars observed close in time to the GRB can be used as a proxy for the atmospheric condition during the GRB observation. Here we employ the telluric correction method that has been developed in Selsing et al. (2015), where a library of synthetic templates is fit to the observed telluric standard.

In the last case, where the object is neither bright enough, or there for some reason a telluric standard have not been observed, we rely on a synthetic sky model (Noll et al. 2012; Jones et al. 2013) for which we generate a synthetic transmission spectrum, where the ambient parameters for the observations have been used.

The effect of telluric correction varies greatly depending on the wavelength region. In the red and near-infrared ranges, telluric correction allows to identify features in a credible manner, which would be otherwise hidden among or blended with atmospheric features. The telluric correction also allows us to reliably recover the continuum level even in regions of moderately low atmospheric transmission (see, for example the $A$ - and $B$-bands in Fig. 4). Nonetheless, we recommend caution when fitting lines in regions significantly affected by telluric absorption.

\subsection{Continuum estimate}

We additionally provide an estimate of the continuum for all the spectra presented here. For this, we have developed an algorithmic approach that attempts to automatically estimate the continuum placement along with the error on the continuum estimate through an iterative procedure. The method is entirely data-driven and does not rely on any physical assumptions. The method is applied on each arm separately for each spectrum, to allow the widest possible wavelength range of the spectral shape to guide the normalization.

To estimate the continuum, a number of points (typically on the order of 100) are inserted at random positions along the wavelength direction, and the flux-density of each point is determined by the median value of the spectrum in a small region $(\sim 1 \AA)$ surrounding each point. The points are fitted with a low order polynomial (we use numpy.polynomial.chebyshev) and iteratively, the point furthest away is removed until the polynomial fit differed from the points by less than $\sim 5$ per cent. This filtering is used because the intrinsic afterglow continuum of GRBs can be modelled by power laws (Piran 2005), and removing points that differ significantly from a smooth continuum shape will guide the continuum estimate to a shape more reasonable for GRB afterglows. The reason for the non-physical model for the normalization is that it has the flexibility to capture instrumental variations of the continuum level that are not easily contained in a more physically motivated model. Additionally, points spaced closer than 1 per cent of the total spectral coverage are pruned. The remaining filtered and pruned points are then spline interpolated using scipy.interpolate.splrep.splev, which serves as a first

\footnotetext{
13 http: //www . eso.org/sci/software/pipelines/skytools/
} molecfit 


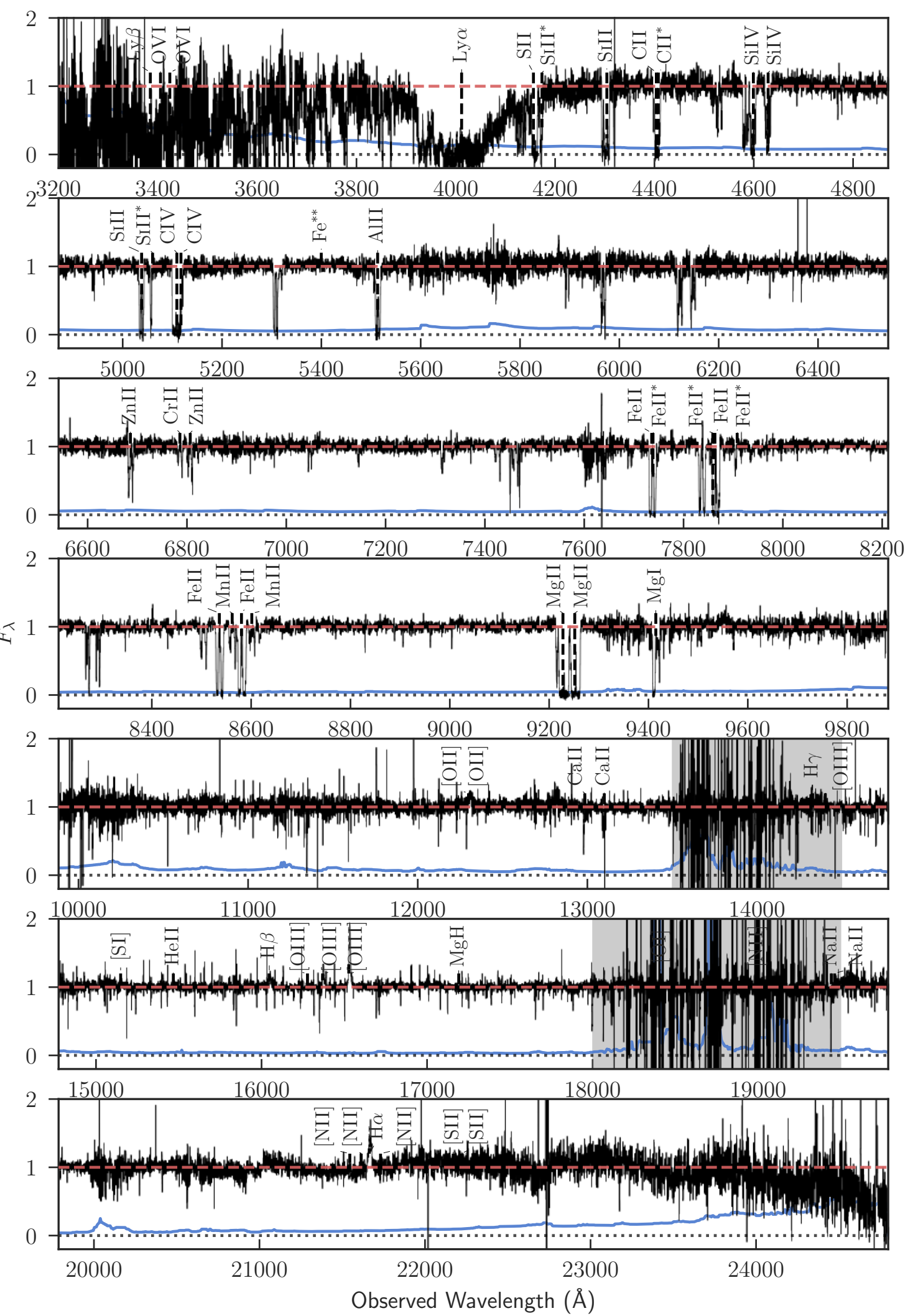

Fig. 4. Telluric corrected, normalised spectrum of GRB 121024A at $z=2.300$ that illustrates the typical data quality. The continuum estimate is shown in dashed red and the error spectrum in solid blue. The acquisition magnitude is $R=20$, meaning it is in the brighter end of the sample presented here, but not the brightest. The spectrum is rich in absorption and emission lines, including absorption from molecular $\mathrm{H}_{2}$. The absorption trough visible at $\sim 4000 \AA$ is due to Ly $\alpha$ in the host. We have marked the most prominent lines seen in GRB afterglows from Christensen et al. (2011). The regions of most severe telluric absorption are highlighted by grey-shading the background. Additionally, three intervening systems are seen in this sightline. This spectrum has been analysed in detail in Friis et al. (2015). 
estimate of the continuum placement. To prevent the spline from diverging at the edges, the spline-based continuum is tapered with a low order polynomial. An attempt to identify absorption and emission regions is then carried out, where they are marked as such if the difference between the estimated continuum and the observed spectrum is larger than 3-5 times the associated error spectrum. All regions marked as affected are then masked.

\subsection{Science data products}

All the spectra are made available as a single ZIP file ${ }^{14}$, (de Ugarte Postigo et al. 2014a), and additionally through the ESO archive in the form of phase 3 material ${ }^{15}$. This release includes both prompt afterglow observations as well as late time observations of the associated hosts, and represents all afterglow spectra of GRBs carried out by the X-shooter spectrograph since the commissioning of the instrument, 14/03/2009, and until the end of the last period of the programme 098.A-0055, $31 / 03 / 2017$ and thus constitutes eight years of GRB afterglow observations with X-shooter. An overview of all the spectra and their observational setups is given in Table A.1. For each burst, each individual observation is provided in a separate reduction, and in cases where observations have been repeated for an increased $\mathrm{S} / \mathrm{N}$ or to follow the temporal evolution, a combined spectrum is also provided. No attempt has been made to join the spectroscopic arms, so for each observation, three spectra are provided in separate files.

All spectra are released in the ESO Science Data Product (SDP) format (Micol et al. 2016), and formatted as binary FITS files. The naming convention is based on the GRB name and the observation number, and follow the scheme GRBXxxxxxx_OBxarm.fits. For example, the visual arm of the third observation of GRB 151021A, observed in RRM mode (see Sect. 2.3), is named GRB151021A_OB3VIS.fits.

Each file contains 7 columns with the following contents and descriptions:

- WAVE - Observed wavelength in vacuum, corrected for barycentric motion and drifts in the wavelength solution $(\AA)$.

- FLUX - Observed flux density ( $\mathrm{erg} \mathrm{s}^{-1} \mathrm{~cm}^{-2} \AA^{-1}$ ).

- ERR - Associated flux density error $\left(\operatorname{erg~s}^{-1} \mathrm{~cm}^{-2} \AA^{-1}\right)$.

- QUAL - Bad pixel map, where a value different from zero indicates a bad pixel.

- CONTINUUM - Continuum estimate based on Sect. 3.7 $\left(\operatorname{erg~s}^{-1} \mathrm{~cm}^{-2} \AA^{-1}\right)$.

- CONTINUUM_ERR - Relative error on the continuum estimate.

- TELL_CORR - Inverse transmission spectrum. Multiply FLUX and ERR column with this column to correct for telluric absorption.

\section{Results}

In this section, we describe the efficiency of the follow-up effort and the characteristics of the observed bursts. We also assess the degree to which the obtained sample is representative for the full Swift sample. An important note is that here we provide a release for all GRBs after 14/03/2009, that have been observed with X-shooter, while only a subset of these constitutes our statistical sample. The statistical sample is based on the selection criteria described in Sect. 2.1. Some bursts not

\footnotetext{
14 http://grbspec.iaa.es

15 http://archive.eso.org/wdb/wdb/adp/phase3_main/form
}

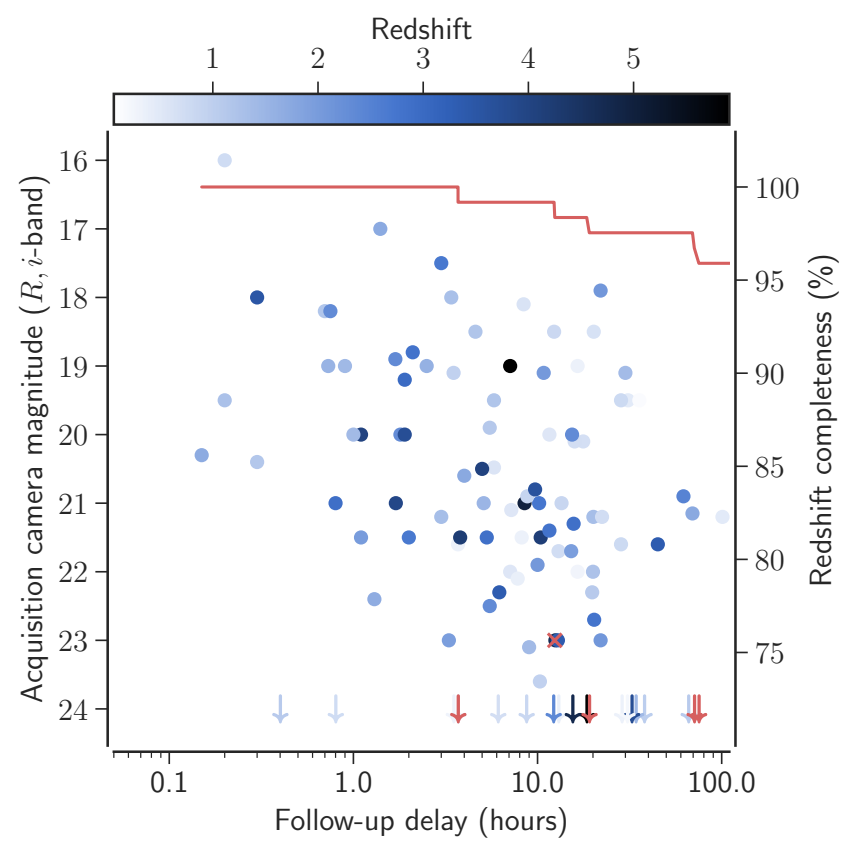

Fig. 5. Afterglow magnitude at the start of observation and redshift completeness as a function of follow-up delay for all the afterglows that have been followed up. The points have been coloured based on the redshift of the corresponding burst. Red symbols indicate GRBs without a measured redshift and arrows indicate bursts for which the afterglow was not detected in the acquisition image. In red is shown the redshift completeness as a function of follow-up delay.

fulfilling the sample criteria have been followed up due to interesting characteristics, due to curious properties of their light curves, their brightness, etc. These bursts are not discussed as part of the investigation of the statistical properties of the GRB population. A prime example of a spectacular burst outside the statistical sample is the bright INTEGRAL burst GRB 161023A (de Ugarte Postigo et al. 2018), that contains at least 15 intervening absorption systems (see Appendix C.117).

\subsection{Follow-up timing and afterglow brightness}

Redshift determination of bursts for which the host is too faint for a spectroscopic redshift measurement relies on the detection of absorption lines imprinted on the GRB afterglow continuum. Because the optical afterglow rapidly fades (typically as $\sim t^{-1}$ ) a rapid follow-up is essential. In Fig. 5 we plot the delay from the BAT trigger to the start of the spectroscopic observation. The shortest delays are observed in RRM-mode. The fastest followup between BAT trigger and start of spectroscopic observations for any observation is for the short, $z=1.717$, GRB 160410A for which spectroscopic integration was initiated only $8.4 \mathrm{~min}$ after the BAT trigger. To illustrate the importance of the follow-up delay for the redshift completeness, we plot the redshift completeness as a function of delay time in Fig. 5 for all the bursts we have followed up, including the ones outside the statistical sample. As can be seen from the figure, the fraction of GRBs with a redshift determination decreases with follow-up delay. The redshift completeness for bursts that we have followed up is 94 per cent. This degree of completeness in the followed bursts, illustrates the efficiency of VLT/X-shooter in redshift determination. Not shown in the figure are an additional 12 bursts that have redshift determinations based on late-time host observations with delay times longer than $\sim 10$ days. 


\subsection{Sample completeness}

Of all the BAT-triggered bursts, a total of 165 bursts fulfill the sample criteria specified in Sect. 2.1, since the commissioning of VLT/X-shooter. This sample constitutes the statistical sample from which we will derive statistical properties of the GRB population. The redshift completeness of the full statistical sample is 61 per cent. We return to the question of redshift completeness in Sect. 4.3. From this sample, 93 GRBs have been spectroscopically followed up with X-shooter. In order to assess whether the subset of bursts followed up are representative of the underlying GRB parent population, we compare intrinsic properties of GRBs in our sample to GRBs in the full sample followed up by Swift. We show the comparison between the BAT (15-150 keV) fluence, the XRT flux (0.3-10 keV) at 11 hours, and the intrinsic $\mathrm{X}$-ray derived equivalent hydrogen column density at the redshift of the GRB, in excess of the Galactic X-ray absorption column, $N_{\mathrm{HI}, \mathrm{X}}$, in Fig. 6 . For the latter, we can only use values of $N_{\mathrm{HI}, \mathrm{X}}$ derived for bursts with a measured redshift, excluding $\sim 75$ per cent of the full Swift sample. We return to the last point in Sect. 4.4.

Using the observational characteristics of the 1266 bursts observed until $31 / 03 / 2017$ by Swift, and the derived $N_{\mathrm{HI}, \mathrm{X}}$ (Evans et al. 2009), we can quantify the degree to which our sample is biased relative to the overall Swift sample. The values are queried from the online Swift database ${ }^{16,17}$. Three samples are of interest in order to assess the completeness of the followup campaign (Fig. 6); the full Swift sample consisting of all the bursts observed by Swift (blue), all the bursts that fulfill the selection criteria imposed in Sect. 2.1 (green), and the bursts actually followed up with X-shooter (red).

For each of the samples, we calculate the median, 16th, and 84th percentiles of each of the distributions, which can be used as point estimates for the population distribution. These are provided in Table 1. It can be seen from the values that the three samples have very similar distributions in terms of the point estimates chosen. This suggests that our selection criteria are unbiased compared to the Swift-sample and that additionally, the follow-up effort conserves the distributions of the intrinsic GRB properties (except perhaps for $N_{\mathrm{HI}, \mathrm{X}}$, see Sect. 4.4).

Additionally, using a 2-sided Kolmogorov-Smirnov test (KS-test), we can assess the degree to which the null hypothesis, that the two distributions are drawn from the same parent distribution, can be rejected. We show a graphic representation of the test statistics in Fig. 7. A high $p$-value indicates little evidence against the null hypothesis. The distribution of $N_{\mathrm{HI}, \mathrm{X}}$ exhibits the highest degree of dissimilarity, but the two distribution are still consistent with being drawn from the same underlying distribution.

We therefore conclude that the statistics of the sample presented here, conserves the intrinsic properties of the GRBs in the full Swift sample - at least in terms of BAT fluence and X-ray flux at $11 \mathrm{~h}$.

Out of the 165 bursts meeting our initial selection criteria for the statistical sample, 36 ( 22 per cent) were not observed due to reasons unrelated to the GRB or afterglow properties. The reasons include unavailability of the telescope due to technical maintenance (because of mirror re-coating), a visiting observer rejecting the ToO trigger, or bad weather. Because this cut is unrelated to the GRB properties, it will not change the statistical properties of the full sample. Removing these bursts from the statistical sample, dramatically improves the redshift com-

\footnotetext{
16 http://swift.gsfc.nasa.gov/archive/grb_table/

17 http://www.swift.ac.uk/xrt_live_cat/
}

pleteness from 61 per cent to 88 per cent. The remaining burst not followed up already had a redshift from other instruments or were very faint and without a host association, thus observations were unlikely to yield a redshift measurement. In the remainder of the text, we consider the 129 bursts our statistical sample.

\subsection{On the redshift distribution of GRBs}

One of the objectives of our follow-up campaign is to measure the redshift distribution for a well-defined, observationally unbiased and statistically useful sample of GRBs. The imposed selection criteria (see Sect. 2.1) ensure that the GRBs entering our homogeneously selected sample, fairly represent the underlying population. The redshift distribution of such a sample holds valuable information about the occurrence of GRBs through cosmic time (Jakobsson et al. 2012; Perley et al. 2016a).

A possible complication is that a large fraction of the redshifts are measured from absorption systems, which in principle only provide lower limits to the GRB redshift. While for individual systems we cannot rule out a low-column density host which is missed, especially in low S/N spectra (see for example the discussion by Perley et al. 2008, 2017a), we argue that this issue is very unlikely to affect significantly our sample.

First, numerous works, including this one, have found that GRB absorption systems have much higher column densities than randomly selected sight lines (Galama \& Wijers 2001; Savaglio et al. 2003; Prochaska et al. 2007; Fynbo et al. 2009). This fact itself shows that the bulk of GRB absorption lines are not due to random intervening systems, but are peculiar to the GRB host. A second line of evidence comes from the systematic detection of nebular emission lines at the same redshift as the absorption system. Krühler et al. (2015a) report a 94\% detection rate of emission lines at $z<1$ (where sensitivity is not an issue, even in the presence of a bright afterglow), always in agreement with absorption. Besides, it is rare to detect emission from QSO intervening systems (see e.g. Fynbo et al. 2010 for a discussion), which again indicates that GRB absorption systems are native to their hosts. An even more direct determination comes from the detection, in several cases, of finestructure and metastable lines (for example from $\mathrm{Fe}$ and $\mathrm{Ni}$ ), which are usually detected in GRB environments (and have been directly connected to the UV field generated by the GRB itself; e.g. Prochaska et al. 2006; Vreeswijk et al. 2007; D'Elia et al. 2009). Finally, at $z \gtrsim 2$ (where Ly $\alpha$ enters the observed range) the onset of the Ly $\alpha$ forest also provides confirming evidence of the GRB redshift, which again is always consistent with the absorption line redshift. The whole set of these arguments indicates that in only very few cases, if any at all, is a misidentification of the redshift with the intervening system a concrete possibility.

In Fig. 8 we show the redshift distribution of all the observed GRBs. In the top panel we show a histogram for the full sample and the statistical sample and in the main panel, we show the redshifts of the individual bursts as a function of GRB energy in the observed $15-150 \mathrm{keV}$ band. To calculate the energy, $E_{\mathrm{BAT}}$, we follow a similar procedure as Lien et al. (2016) and define $E_{\mathrm{BAT}}=F_{\gamma} 4 \pi d_{\mathrm{L}}^{2}(1+z)^{-1}$, where $F_{\gamma}$ is the observed BAT fluence in the $15-150 \mathrm{keV}$ band and $d_{\mathrm{L}}$ is the luminosity distance to the burst at the given redshift. We note that this measure of luminosity does not include any $k$-correction. As an indication of the effect of the Swift sensitivity limit on the redshift distribution, also in the figure, we have shown the so called $\sim 1$ s flux 

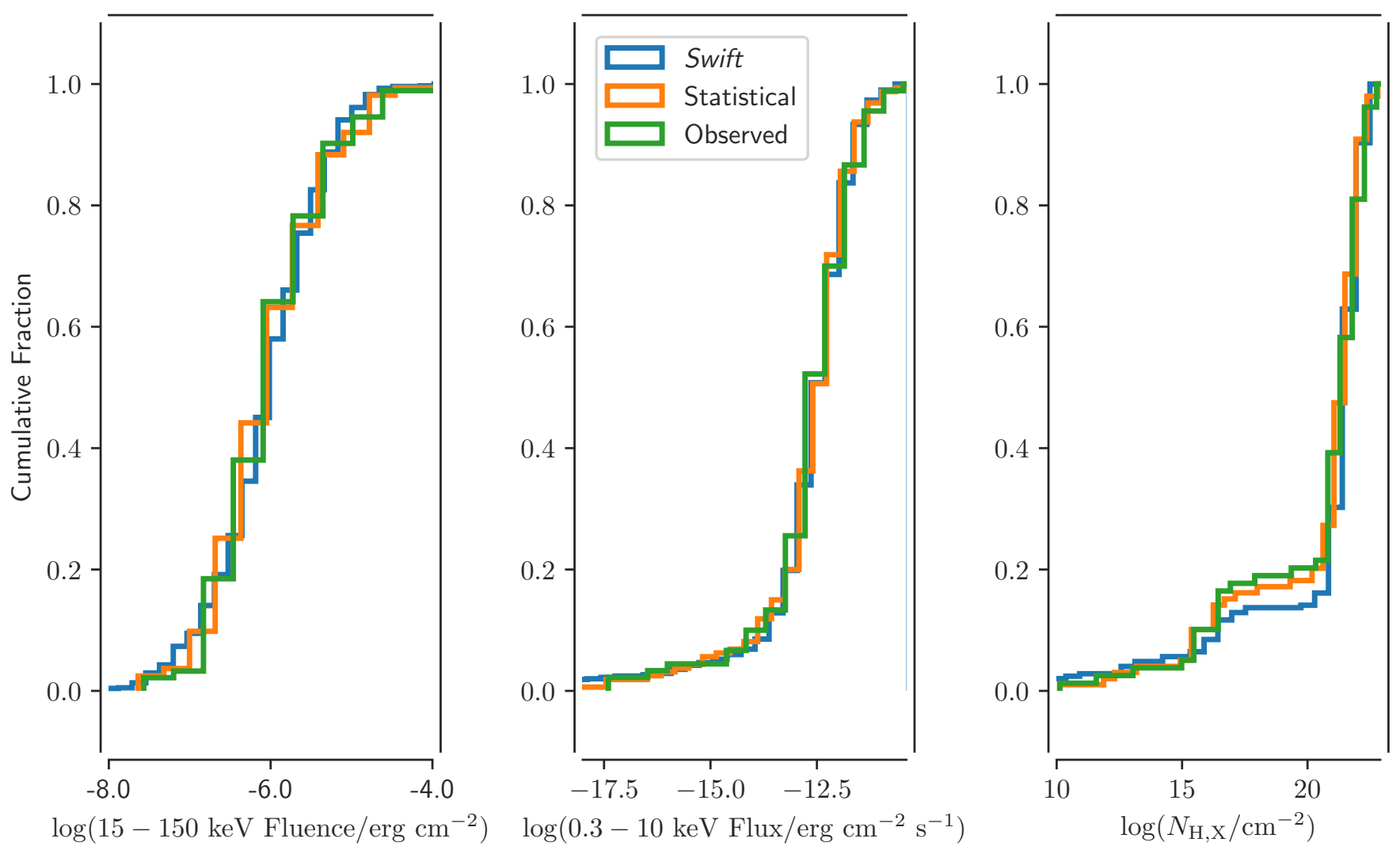

Fig. 6. Comparison between the burst properties of all bursts observed with Swift (blue), the statistical sample that fullfill the criteria specified in Sect. 2.1 (green), and the subset that has been observed as part of the statistical sample (red). Left panel: fluence in the $15-150 \mathrm{keV}$ band as observed by BAT. Middle panel: $0.3-10 \mathrm{keV}$ flux, $11 \mathrm{~h}$ after the bursts as measured by XRT. Right panel: $N_{\mathrm{HI}, \mathrm{X}}$ based on the XRT spectrum (Evans et al. 2009).

Table 1. Population properties (median and 16th and 84th percentiles as the error intervals) for the Swift sample and the subset of bursts fulfilling the sample criteria.

\begin{tabular}{cccc}
\hline \hline & Full Swift sample & Statistical sample & Followed up bursts \\
\hline$N_{\mathrm{BAT}}$ & 981 & 163 & 92 \\
$\log (15-150 \mathrm{keV}$ fluence $)$ & $-5.9_{-0.6}^{+0.7}$ & $-5.9_{-0.6}^{+0.7}$ & $-5.9_{-0.7}^{+0.7}$ \\
$N_{\mathrm{XRT}}$ & 902 & 160 & 90 \\
$\log (0.3-10 \mathrm{keV}$ flux $)$ & $-12.3_{-0.8}^{+0.7}$ & $-12.4_{-0.8}^{+0.7}$ & $-12.4_{-0.7}^{+0.9}$ \\
$N_{\mathrm{HI}_{x}}$ & $248^{-0.8}$ & $99^{-0.7}$ & $79^{+0.7}$ \\
$\log \left(N_{\mathrm{HI}_{x}}\right)$ & $21.7_{-0.9}^{+0.6}$ & $21.5_{-3.4}^{+0.7}$ & $21.6_{-4.5}^{+0.7}$ \\
\hline
\end{tabular}

Notes. The population characteristics of the three samples are very similar, which shows that our selection criteria effectively conserve the statistical properties of the underlying population, as least for these parameters. Notice that not all bursts have measurements of the quantities we compare.

BAT sensitivity limit $\left(\sim 3 \times 10^{-8} \mathrm{erg} \mathrm{s}^{-1} \mathrm{~cm}^{-2}\right.$; Baumgartner et al. 2013; Lien et al. 2016). Due to the complex triggering mechanism of Swift, this sensitivity limit should be interpreted with some caution as the effective limit depends on the light curve of the prompt emission signal. Due to the dilution of light with distance, the Swift GRB luminosity detection limit is almost an order of magnitude brighter at $z=2$ than at $z=1$. At $z \geq 4$ we are only able to observe GRBs that are $\sim$ hundred times brighter than the faintest bursts at $z=1$ and below. The effect of GRB redshift on the Swift triggering criteria have previously been studied in detail (Littlejohns et al. 2013).

We compare the point estimates for the redshift distributions of previous complete samples of GRBs in Table 2. We see that when we compare to other complete samples, the XS-GRB presented here has the lowest average redshift. However, the other samples also exhibit a large spread in the redshift distributions. A 2-sided KS test reveals that the XS-GRB sample is consistent with being drawn from the same parent sample with the following $p$-values: SHOALS ( $p$-value $=0.13)$, BAT6 $(p$-value $=0.95)$, Fynbo09 $(p$-value $=0.08)$ and TOUGH $(p$-value $=0.09)$. As a small note, the redshift distribution of BAT6 are not expected to be identical to the other complete samples due to the additional cut on the GRB peak flux in the BAT band.

Because the redshift completeness of our statistical sample is 88 per cent, making an inference of the true redshift distribution of GRBs based on this sample is impossible. For instance, only the brightest GRBs are seen above redshift $z \gtrsim 1$ as shown in Fig. 8. As described in detail in Hjorth et al. (2012) and Perley et al. (2016b), bursts for which the redshift is measured from the afterglow are systematically found in host galaxies with a lower 


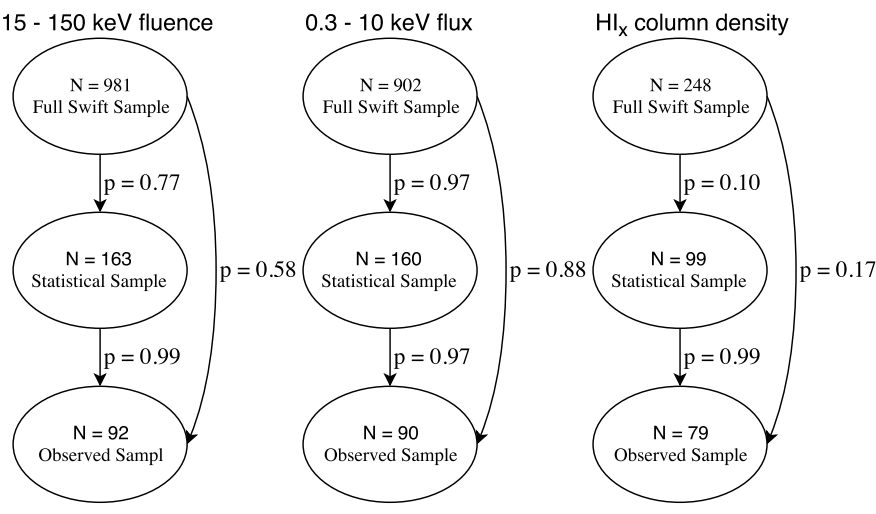

Fig. 7. Relational graph showing the respective $p$-values. They all represent the degree that the different samples are drawn from the same underlying distribution. The arrows represent the comparison direction with each of the $p$-values they are listed next to. Only in the HI column density distribution is there mild evidence against the null hypothesis, but the discrepancy is mainly driven by a relatively larger fractional contribution from low-column hosts in the statistical sample.

luminosity than bursts for which the redshift is measured from the host galaxy. Only a few GRBs hosted in galaxies, with stellar masses more than $10^{10} M_{\odot}$ have the redshift measured based on the afterglow continuum. This is likely related to the presence of higher contents of dust in more massive galaxies, leading to a larger fraction of extincted afterglows.

\subsection{Sample darkness}

A fraction of all GRBs exhibit no detectable or very faint optical afterglows (Groot et al. 1998; Djorgovski et al. 2001; Fynbo et al. 2001). The degree of optical extinction relative to the X-ray brightness has been parametrised in terms of their optical darkness, using the measurement of, or limit on, the optical to X-ray spectral index $\beta_{\mathrm{OX}}$ (Jakobsson et al. 2004; Rol et al. 2005; van der Horst et al. 2009). The X-ray properties of such bursts have previously been investigated (De Pasquale et al. 2003; Fynbo et al. 2009; Melandri et al. 2012) and there are some indications that dark bursts have somewhat higher X-ray luminosity and $N_{\mathrm{H}, \mathrm{X}}$ compared to the optically bright bursts (Campana et al. 2012; Watson \& Jakobsson 2012). The X-ray column density has been shown to be roughly correlated with the gas column density, which, for a given metallicity, also correlates with the dust extinction (Watson et al. 2013; Covino et al. 2013), though the range in metallicity introduces a large additional scatter in the correlation between the extinction and the $N_{\mathrm{H}, \mathrm{X}}$. This indicates along with investigations of host galaxy properties (Greiner et al. 2011; Krühler et al. 2011; Hjorth et al. 2012; Perley et al. 2016b), that the extinction of the optical afterglows is primarily driven by the presence of dust in the host galaxies and not solely by unfortunate placement of the synchrotron spectral break frequencies. Hjorth et al. (2012) find that systems with no optical afterglow have higher $N_{\mathrm{H}, \mathrm{X}}$, irrespective of the nature of the host - which however also turn out to be redder. Additionally, the ISM absorption lines in dark sight-lines are found to be stronger compared to optically brighter bursts (Christensen et al. 2011), which is consistent with the dark bursts being found in more metal-rich and dustier galaxies.

For all bursts with follow-up within $100 \mathrm{~h}$ we calculated the "darkness"-parameter, $\beta_{\mathrm{OX}}$ (Jakobsson et al. 2004). This requires the simultaneous measurement of the X-ray flux density and the optical flux density which is in practice possible, but in reality
Table 2. Comparison between the redshift distributions of previous complete samples.

\begin{tabular}{ccccc}
\hline \hline Sample & $N_{\text {bursts }}$ & $z_{\text {completeness }}$ & $z_{\text {mean }}$ & $z_{\text {med }}$ \\
\hline XS-GRB & 129 & $88 \%$ & 1.89 & $1.52_{-0.91}^{+1.83}$ \\
SHOALS & 119 & $99 \%$ & 2.18 & $2.06_{-1.20}^{+1.27}$ \\
BAT6 & 58 & $97 \%$ & 1.90 & $1.71_{-1.04}^{+1.31}$ \\
TOUGH & 69 & $87 \%$ & 2.20 & $2.11_{-1.46}^{+1.42}$ \\
Fynbo09 & 146 & $49 \%$ & 2.2 & $2.1_{-1.23}^{+1.28}$ \\
\hline
\end{tabular}

Notes. The SHOALS redshift characteristics are taken from Perley et al. (2016a), the BAT6 redshifts are from Salvaterra et al. (2012) with the update from Pescalli et al. (2015), TOUGH is from Hjorth et al. (2012) with the update from Schulze et al. (2015), and Fynbo09 are from Fynbo et al. (2009). The errors shown on the median redshift contain 68 per cent of the probability mass.

extremely rarely available. As a proxy, we use the measured acquisition camera magnitude reported in Table A.1 to get the optical flux density at the beginning of the spectroscopic integration. Because we know the delay between the follow-up and the Swift trigger, we can use the measured XRT lightcurve (Evans et al. 2007, 2009) to infer the corresponding X-ray flux density at the time of the optical observation. This is done by either linearly interpolating between temporally neighboring XRT measurements or by extrapolating the last few X-ray data points to the time of the spectroscopic observation. When the afterglow is not detected in the acquisition camera, an upper limit of $>24$ mags is used, which propagates into an upper limit on $\beta_{\mathrm{OX}}$.

In Fig. 9 we compare the $\beta_{\mathrm{OX}}-N_{\mathrm{H}, \mathrm{X}}$ distribution with the one presented in Fynbo et al. (2009). We take the $N_{\mathrm{HI}, \mathrm{X}}$ values from the XRT spectral fits ${ }^{18}$ (Evans et al. 2009). The values from Fynbo et al. (2009) have been treated as detections, meaning that we artificially bias the distribution towards higher $\beta_{\mathrm{OX}}$-values. The two distributions exhibit a large degree of overlap. We confirm the result by Fynbo et al. (2009), that dark bursts, $\beta_{\mathrm{OX}}<0.5$, have higher $\log \left(N_{\mathrm{H}, \mathrm{X}} / \mathrm{cm}^{-2}\right)$. Specifically, for bursts with measured redshift either from the afterglow or the host galaxy, we find the following: For bursts with $\beta_{\mathrm{OX}} \geq 0.5$ we find $\log \left(N_{\mathrm{H}, \mathrm{X}} / \mathrm{cm}^{-2}\right)=21.4_{-1.0}^{+0.7}$, whereas for $\beta_{\mathrm{OX}}<0.5$ we find $\log \left(N_{\mathrm{H}, \mathrm{X}} / \mathrm{cm}^{-2}\right) 21.8_{-0.9}^{+0.5}$ where 68 per cent of the probability mass is contained within the error intervals. A 2-sided KS test fails to reject the null hypothesis that they are drawn from the same distribution with $p=0.11$, meaning that there is no strong evidence for a discrepancy. A Kendall's $\tau$ test, however, suggest a statistically significant, low degree of negative correlation $(\Gamma=-0.21$ at a $p$-value $=0.01)$.

Using the table maintained by J. Greiner ${ }^{19}$, we can see how the presence of an optical afterglow affects the follow-up statistics. 50.5 per cent of all Swift-triggered bursts in this list do not have a detected optical afterglow. This number also includes bursts where no optical observations were available, so the real number is likely to be lower. For the bursts that enter our statistical sample, the percentage of bursts without a detected optical afterglow is 28 per cent, close to the upper limit on the fraction of dark bursts found in a complete sample with a very high degree of redshift completeness (Melandri et al. 2012). Of the bursts for which follow-up has actually been attempted, this number is 23 per cent, suggesting a slight bias against

\footnotetext{
18 http://www.swift.ac.uk/xrt_spectra/

19 http://WWW.mpe.mpg.de/ jcg/grbgen.html
} 


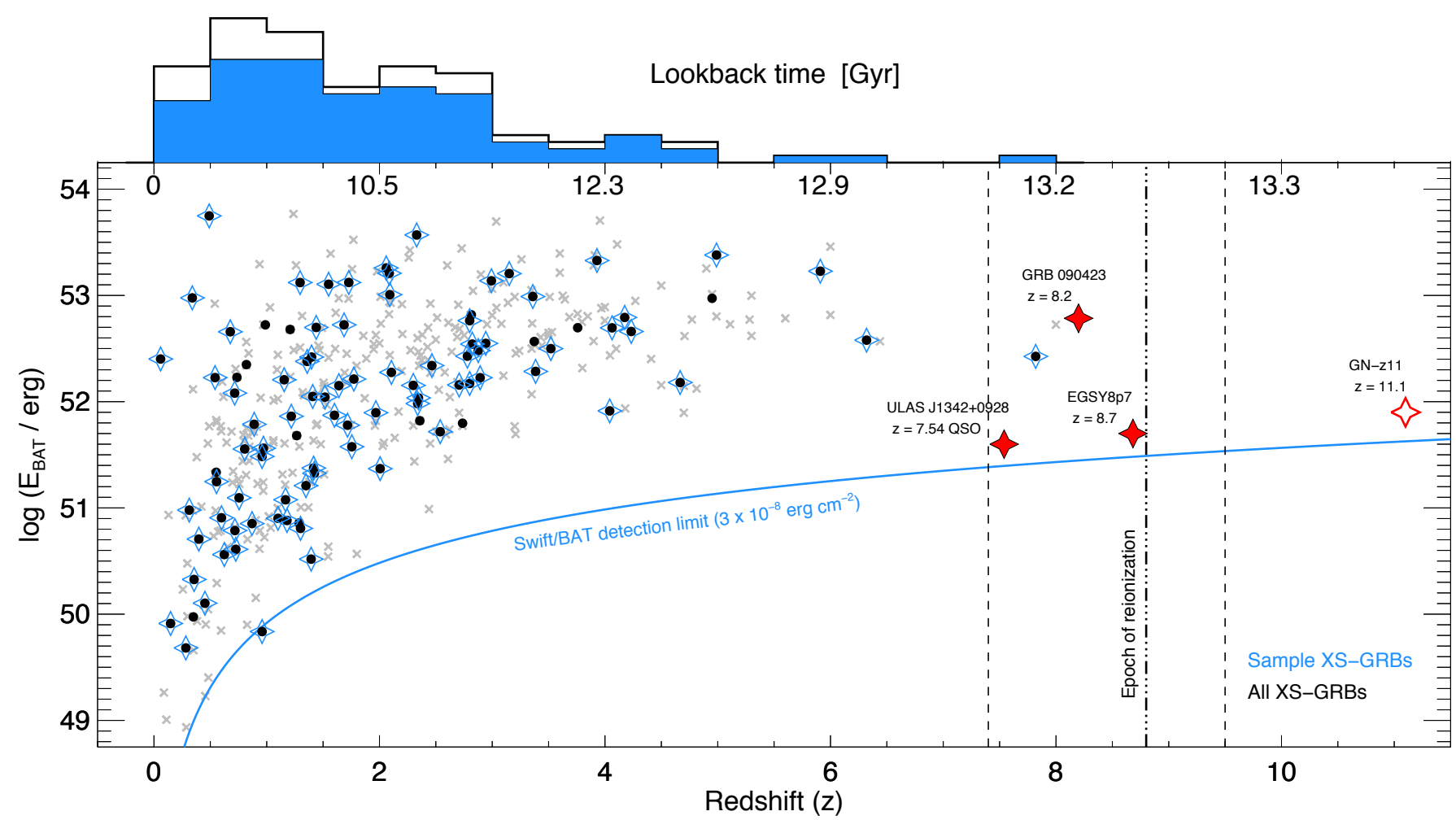

Fig. 8. Redshift distribution as a function of intrinsic BAT $\gamma$-ray energy, $E_{\mathrm{BAT}}$. Bursts that are a part of the statistical sample are marked by blue stars whereas black dots show all GRBs observed with X-shooter. All Swift GRBs with measured redshifts are shown in grey. For comparison, we overplot with red stars the GRB (Tanvir et al. 2009; Salvaterra et al. 2009), quasar (Bañados et al. 2017), and galaxies (Zitrin et al. 2015; Oesch et al. 2016) with the highest spectroscopically confirmed redshifts, the latter three shown at arbitrary $E_{\mathrm{BAT}}$. The blue solid line represents the so-called $1 \mathrm{~s}$ BAT sensitivity limit described in the text. The estimated epoch of reionization is shown by the black dot-dashed line, with the uncertainty shown as the black dashed lines, from the most recent measurement by Planck Collaboration XIII (2016). On the top of the plot is shown the marginalization of the redshift. Again the blue histogram represents the bursts than enter our sample and the white histogram the full GRB population.

bursts without a detected optical afterglow in the spectroscopic sample.

However, the fraction of dark bursts for which we have measured redshifts is lower than the ones with a detected optical afterglow. For the afterglows we have observed as part of the statistical sample that do not have a detected optical afterglow, the redshift completeness is 53 per cent. For comparison, for the afterglows in the statistical sample we have observed with an optical afterglow detected, the redshift completeness is 92 per cent. This also shows that the lack of redshift completeness in the sample presented here is in part due to the increased difficulty of measuring a redshift for bursts without an afterglow. To measure a redshift, we either need a detected afterglow to obtain a spectrum or to locate the host galaxy and determine the redshift from there. It is more difficult to correctly associate a galaxy with a burst when there is no detected optical afterglow and hence a correct redshift measurement is more difficult to make, (see Jakobsson et al. 2005; Levesque et al. 2010 and Perley et al. 2017b).

Regardless the fraction of dark bursts being lower in the observed sample, compared to the statistical sample, the X-ray properties do not differ significantly, as shown in Sect. 4.2. This is despite spectroscopic follow-up only being carried out in cases where either a detectable optical afterglow or a clear counterpart are seen, which naively should be biased against dark bursts occurring in more obscured galaxies, which is shown to exhibit different galactic properties (Perley et al. 2009, 2013, 2015; Krühler et al. 2011; Rossi et al. 2012). That the decreased fraction of dark bursts in observed sample does not alter the observed prompt X-ray brightness distribution, poten- tially reflects the independence of the X-ray brightness on the density of the circumburst medium (Freedman \& Waxman 2001; Berger et al. 2003; Nysewander et al. 2009), if the measured $N_{\mathrm{H}, \mathrm{X}}$ is primarily driven by the gas column in the neighborhood of the burst. Because we only use values for $N_{\mathrm{H}, \mathrm{X}}$ in the comparison for which the GRB has a measured redshift, this measure is likely biased towards optically brighter bursts (e.g. Watson \& Jakobsson 2012).

\subsection{Hydrogen column densities}

The locations of long GRBs are associated with intensely starforming regions (Hogg \& Fruchter 1999; Bloom et al. 2002; Fruchter et al. 2006; Lyman et al. 2017). Because a significant fraction of the hydrogen along the line of sight in these regions has not been ionised, the optical depth at the wavelength of Ly $\alpha$ is very high, saturating not only the line center, but also the damping wings. This causes a strong absorption system from the Ly $\alpha$ transition to appear in the afterglow continuum. For bursts with $z \gtrsim 1.7$, the position of $\operatorname{Ly} \alpha$ moves into the spectroscopic coverage of X-shooter, meaning that we are able to detect this absorption trough due to Ly $\alpha$. The exceptionally good UV response of $\mathrm{X}$-shooter, allows us to robustly measure Hi-columns - also at these relatively low redshifts.

Due to the stochastic nature of the $\operatorname{Ly} \alpha$-forest, the blue wing of the Lyman- $\alpha$ absorption line is randomly superposed with Lyman- $\alpha$ forest systems, along with strong absorption from Mn II and Si III, making it notoriously difficult to model. Additionally, the red wing has ISM signatures imprinted on it, 


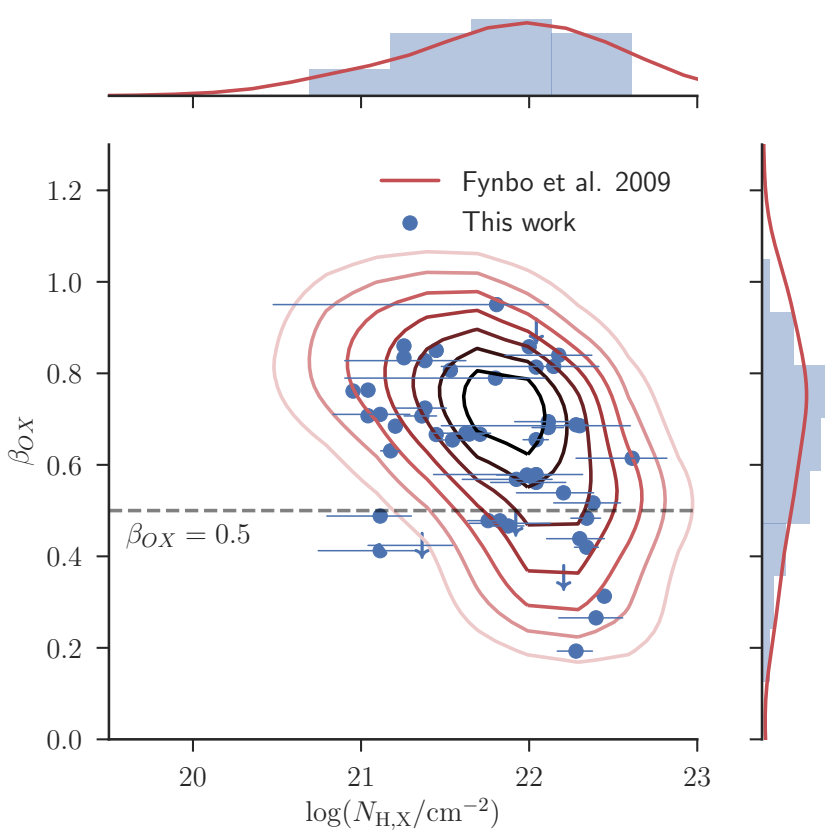

Fig. 9. $\beta_{\mathrm{OX}}$ against $\mathrm{X}$-ray derived hydrogen column density. In red is shown the sample presented in Fynbo et al. (2009) where the lines indicate the kernel density estimate of the distribution. For the kernel density estimate, the limits have been replaced with values. We note that contrary to what is presented in Fynbo et al. (2009), we exclude all bursts for which we do not have a redshift. Darker colours represent a higher density of points. In blue are the points for the bursts presented here along with the marginal histograms. Limits on $\beta_{\mathrm{OX}}$ are shown by downwards facing arrows. The corresponding marginal distributions are shown along the edges of the plot. A Kendall's $\tau$ test, indicates a statistically significant, low degree of negative correlation $(\Gamma=-0.21$ at a $p$-value $=0.01$.

especially strong absorption due to $\mathrm{Si}_{\text {III }} \mathrm{S}_{\text {II }}$ and $\mathrm{N}$ v, which can exhibit significant velocity structure. Along with instrumental effects, the generative model for the data that we would use in a likelihood-based analysis would be very complicated. We have therefore decided not to make formal $\chi^{2}$ fitting of the hydrogen column densities, but instead use a more subjective visual measurement to the absorption profile. Using an analytic approximation to the absorption profile from Tepper García (2006), we overplot a synthetic absorption line with a specified column density on our observed, normalised spectrum. By tuning the value of the hydrogen column density until the synthetic absorption line matches the spectrum, we can thereby infer the actual column density of the GRB sight line in a manual way. Similarly, the uncertainty on the hydrogen column can be estimated by adjusting the error, until the confidence bounds contain the continuum variation. We show the results of this procedure for all bursts where possible in Fig. A.1 and the inferred hydrogen column densities in Table 3.

12 of the $N_{\mathrm{HI}}$ measurements for these spectra have previously been presented in Cucchiara et al. (2015) (see Table 3). We provide new measurements here for completeness. In the compilation of $N_{\mathrm{HI}}$ measurements towards GRBs in Tanvir et al. (2019) there are 93 published $N_{\mathrm{HI}}$ values, excluding the measurements provided here. We here provide 30 new neutral hydrogen column density measurements - an increase of the number of optically derived hydrogen column densities of $\sim 33$ per cent. We show the two distributions in Fig. 10. We compare the median, the 16th, and 84th percentiles of the two distributions. The sample presented has a $\log \left(N_{\mathrm{HI}} / \mathrm{cm}^{-2}\right)=21.8_{-0.8}^{+0.3}$ and the rest of the lit-

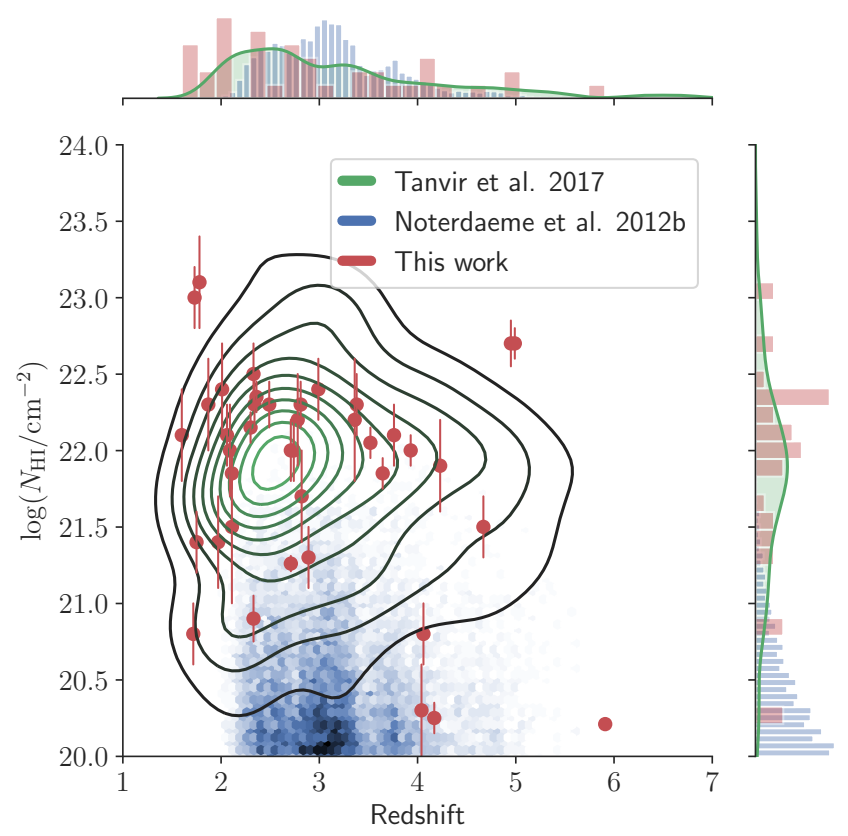

Fig. 10. Distributions of hydrogen column densities for absorbers found in quasar absorption lines, from Noterdaeme et al. (2012) in blue. Overplotted in green is the kernel density estimate of absorbers in GRB sightlines. Values are taken from the compilation in Tanvir et al. (2019), along with the new values presented in this sample. We also show in the red the values derived in this work. The marginal distributions for the three samples are also shown along the left side and on the top, where the different environments probed are clearly visible in the hydrogen column densities, as previously also noted in Fynbo et al. (2009). To correct for geometric differences between GRB DLAs and QSO DLAs, we have multiplied the inferred GRB DLA $N_{\mathrm{HI}}$ by a factor of two.

erature values has $\log \left(N_{\mathrm{HI}} / \mathrm{cm}^{-2}\right)=21.5_{-1.5}^{+0.4}$. We see that the two distributions have a large degree of overlap due to the large width of the distributions, but we find a slightly higher median value for the new sample presented here. A 2 -sided KS test gives a $p$-value of $p=0.006$, meaning relatively strong evidence against the null hypothesis that the two samples are drawn from the same underlying distribution. Because the bursts that have measurements of the hydrogen column density are selected solely based on our ability to infer a column, it is difficult to make any strong conclusions about the population statistics in terms of gas content.

In Fig. 10, we also show the column density distribution for the 12081 quasar absorbers with $\log \left(N_{\mathrm{HI}} / \mathrm{cm}^{-2}\right)>20$ from Noterdaeme et al. (2012). The fact that GRBs are systematically located behind the highest $\log \left(N_{\mathrm{HI}} / \mathrm{cm}^{-2}\right)$, previously noted (e.g. Prochaska et al. 2007; Fynbo et al. 2009), is very clear in this figure. The reason for this is that quasar sample sight-lines through galaxies that are cross-section selected, whereas GRB sight-lines probe the dense, star-forming regions in their hosts. When comparing the GRB DLAs and QSO DLAs we correct for the fact that, on average the GRB light only goes through half of the full ISM gas column. In Fig. 10, we have therefore multiplied the inferred $N_{\mathrm{HI}}$ values by 2 (Prochaska et al. 2008).

\section{Discussion and conclusions}

In this paper we have presented the results of a dedicated effort over the years 2009-2017 to use the X-shooter spectrograph on the ESO-VLT to secure spectroscopic observations of afterglows and host galaxies of GRBs detected by Swift. This work was initiated by the consortium that built X-shooter and included this 
Table 3. Hydrogen column densities for the 41 bursts exhibiting Ly $\alpha$ absorption in the spectral coverage of X-shooter.

\begin{tabular}{|c|c|}
\hline GRB & $\begin{array}{l}\text { Hydrogen column } \\
\log \left(N_{\mathrm{HI}} / \mathrm{cm}^{-2}\right)\end{array}$ \\
\hline GRB 090809A ${ }^{c}$ & $21.7 \pm 0.2$ \\
\hline GRB 090926A & $21.55 \pm 0.10$ \\
\hline GRB $100219 A^{c}$ & $21.2 \pm 0.2$ \\
\hline GRB $100425 \mathrm{~A}^{c}$ & $21.0 \pm 0.2$ \\
\hline GRB 100728B & $21.2 \pm 0.5$ \\
\hline GRB 110128A & $21.90 \pm 0.15$ \\
\hline GRB 110818A & $21.9 \pm 0.4$ \\
\hline GRB $111008 \mathrm{~A}^{c}$ & $22.40 \pm 0.10$ \\
\hline GRB $111107 \mathrm{~A}^{c}$ & $21.0 \pm 0.2$ \\
\hline GRB 120119A & $22.6 \pm 0.2$ \\
\hline GRB $120327 A^{c}$ & $22.00 \pm 0.05$ \\
\hline GRB 120404A & $20.7 \pm 0.3$ \\
\hline GRB 120712A & $19.95 \pm 0.15$ \\
\hline GRB $120716 A^{c}$ & $21.80 \pm 0.15$ \\
\hline GRB $120815 A^{c}$ & $22.10 \pm 0.10$ \\
\hline GRB $120909 \mathrm{~A}^{c}$ & $21.75 \pm 0.10$ \\
\hline GRB $121024 A^{c}$ & $21.85 \pm 0.10$ \\
\hline GRB 121027A & $22.8 \pm 0.3$ \\
\hline GRB $121201 \mathrm{~A}^{a, c}$ & $22.0 \pm 0.3$ \\
\hline GRB 121229A & $21.8 \pm 0.2$ \\
\hline GRB $130408 \mathrm{~A}^{c}$ & $21.80 \pm 0.10$ \\
\hline GRB 130427B & $21.9 \pm 0.3$ \\
\hline GRB 130606A & $19.91 \pm 0.05$ \\
\hline GRB 130612A & $22.1 \pm 0.2$ \\
\hline GRB 131011A & $22.0 \pm 0.3$ \\
\hline GRB 131117A & $20.0 \pm 0.3$ \\
\hline GRB 140311A & $22.40 \pm 0.15$ \\
\hline GRB 140430A & $21.8 \pm 0.3$ \\
\hline GRB 140515A & $19.0 \pm 0.5$ \\
\hline GRB 140614A & $21.6 \pm 0.3$ \\
\hline GRB 141028A & $20.60 \pm 0.15$ \\
\hline GRB 141109A & $22.10 \pm 0.10$ \\
\hline GRB 150206A & $21.7 \pm 0.4$ \\
\hline GRB 150403A & $21.8 \pm 0.2$ \\
\hline GRB $150915 \mathrm{~A}^{a}$ & $21.2 \pm 0.3$ \\
\hline GRB $151021 A^{a}$ & $22.3 \pm 0.2$ \\
\hline GRB 151027B & $20.5 \pm 0.2$ \\
\hline GRB 160203A & $21.75 \pm 0.10$ \\
\hline GRB $160410 A^{b}$ & $21.2 \pm 0.2$ \\
\hline GRB 161014A & $21.4 \pm 0.3$ \\
\hline GRB 161023A & $20.96 \pm 0.05$ \\
\hline GRB $170202 \mathrm{~A}^{a}$ & $21.55 \pm 0.10$ \\
\hline
\end{tabular}

Notes. The corresponding measurements are shown in Fig. A.1. ${ }^{(a)} \mathrm{Has}$ Ly $\alpha$ emission in the trough. ${ }^{(b)}$ Short burst. ${ }^{(c)}$ Previously published in Cucchiara et al. (2015).

project as a key part of the GTO programme, but over the years the project continued in open time.

The sample presented here includes spectroscopic observations of 93 systems fulfilling our sample criteria, including 18 spectra that are late-time observations of the underlying host galaxies. All spectra have been made publicly available in the reduced form used in this paper.

Our sample serves the purpose to further characterise the environments of GRBs that was also much advanced by the previous surveys based primarily on lower-resolution spectroscopy. GRB afterglow sight-lines are unique in the sense that only after observing more than 12000 damped Lyman- $\alpha$ absorbers (DLAs) towards about $10^{5}$ quasars, a handful systems with $\log \left(N_{\mathrm{HI}} / \mathrm{cm}^{-2}\right)>22$ have been identified (for instance five in Noterdaeme et al. 2012). Long GRB afterglow spectra, by contrast, reveal such systems routinely (Jakobsson et al. 2006; Fynbo et al. 2009; Cucchiara et al. 2015, and this work). With afterglow spectroscopy (throughout the electromagnetic spectrum from $\mathrm{X}$-rays to the sub-mm) we are able to characterise the properties of star-forming galaxies over cosmic history in terms of redshifts, metallicities, molecular contents, ISM temperatures, UV-flux densities, extinction curves, etc. A number of independent papers have been published or submitted for publication focusing on many of these specific issues of our sample such as extinction curves (Japelj et al. 2015; Zafar et al. 2018a,b, see also Fynbo et al. 2014b; Heintz et al. 2017), emission lines from the underlying host galaxies (Krühler et al. 2015a), the frequency of intervening Mg II absorbers (Christensen et al. 2017), Arabsalmani et al. (2018) on the metallicity-scaling relations, and escape of ionising radiation (Tanvir et al. 2019). A number of additional companion papers are also planned, investigating the detailed properties of the sample presented here, including equivalent width distributions (de Ugarte Postigo et al., in prep.), metallicities and kinematics (Thöne et al., in prep.), high ionization lines (Heintz et al. 2018a), molecular lines (Bolmer et al. 2019), fine-structure lines (Vreeswijk et al., in prep.), and composite GRB afterglow spectrum (Selsing et al., in prep.).

The potential of using GRB sightlines as probes is far from fully harvested. The sample of sightlines probed by our spectra are not representative for all GRB sightlines as we have shown and consistent with earlier findings from samples based on low-resolution spectroscopy (e.g. Fynbo et al. 2009) and from studies of complete samples of GRB host galaxies (Hjorth et al. 2012; Covino et al. 2013; Perley et al. 2016a). Krühler et al. (2013a) argue, that very rich sightlines like that probed by the remarkable GRB 080607 (Prochaska et al. 2009; Sheffer et al. 2009; Perley et al. 2011) are probably significantly more frequent than in the sightlines sampled by our spectra. However, with current instrumentation, these sightlines are out of reach except under very fortunate circumstances as in the case of GRB 080607 when the afterglow could be observed only a few minutes after the burst with a 10-m class telescope. Observations of such sightlines with $\mathrm{X}$-shooter-like spectrographs on the next generation of 20-40-m telescopes is likely to be very rewarding, given that a suitable GRB detector will be available.

Acknowledgements. Much of the work done here would not have been done without Neil Gehrels, who has now passed away. We owe him a large debt for his invaluable work with the Swift satellite. We also want to remember Javier Gorosabel, who was taken from us too early. We thank Andrea Rossi (INAF/OAS) for the help with providing a photometric constraint on the redshift of GRB 161007A. JPUF, BMJ and DX acknowledge support from the ERC-StG grant EGGS-278202. The Dark Cosmology Centre was funded by the Danish National Research Foundation. This work was supported by a VILLUM FONDEN Investigator grant to JH (project number 16599). TK acknowledges support by the European Commission under the Marie Curie Intra-European Fellowship Programme in FP7. LK and JJ acknowledges support from NOVA and NWO-FAPESP grant for advanced instrumentation in astronomy. KEH and PJ acknowledge support by a Project Grant (162948-051) from The Icelandic Research Fund. AG acknowledges the financial support from the Slovenian Research Agency (research core funding No. P1-0031 and project grant No. J1-8136). CT acknowledges support from a Spanish National Research Grant of Excellence under project AYA 2014-58381-P and funding associated to a Ramón y Cajál fellowship under grant number RyC-2012-09984. AdUP acknowledges support from a Ramón y Cajal fellowship, a BBVA Foundation Grant for Researchers and Cultural Creators, and the Spanish Ministry of Economy and Competitiveness through project AYA2014-58381-P. ZC acknowledges support from the Spanish research project AYA 2014-58381-P and 
support from Juan de la Cierva Incorporación fellowships IJCI-2014-21669. DAK acknowledges support from the Spanish research project AYA 201458381-P and support from Juan de la Cierva Incorporación fellowships IJCI-2015- 26153. RSR acknowledges AdUP's BBVA Foundation Grant for Researchers and Cultural Creators and support from ASI (Italian Space Agency) through the Contract n. 2015-046-R.0 and from European Union Horizon 2020 Programme under the AHEAD project (grant agreement n. 654215). GL is sup ported by a research grant (19054) from VILLUM FONDEN. SDV acknowledges the support of the French National Research Agency (ANR) under contract ANR-16-CE31-0003 BEaPro DM acknowledges support from the Instrument Center for Danish Astrophysics (IDA). This work made use of data supplied by the UK Swift Science Data Centre at the University of Leicester. Finally, it is with pleasure that we acknowledge expert support from the ESO staff at the Paranal and La Silla observatories in obtaining these target of opportunity data. This research made use of Astropy, a community-developed core Python package for Astronomy (Astropy Collaboration 2013). The analysis and plotting was achieved using the Python-based packages Matplotlib (Hunter 2007) Numpy, and Scipy (Oliphant 2007; Van Der Walt et al. 2011), along with other community-developed packages. This publication makes use of data products from the Wide-field Infrared Survey Explorer, which is a joint project of the University of California, Los Angeles, and the Jet Propulsion Laboratory/California Institute of Technology, funded by the National Aeronautics and Space Administration. The Pan-STARRS1 Surveys (PS1) and the PS1 public science archive have been made possible through contributions by the Institute for Astronomy, the University of Hawaii, the Pan-STARRS Project Office, the Max-Planck Society and its participating institutes, the Max Planck Institute for Astronomy, Heidelberg and the Max Planck Institute for Extraterrestria Physics, Garching, The Johns Hopkins University, Durham University, the University of Edinburgh, the Queen's University Belfast, the Harvard-Smithsonian Center for Astrophysics, the Las Cumbres Observatory Global Telescope Network Incorporated, the National Central University of Taiwan, the Space Telescope Science Institute, the National Aeronautics and Space Administration under Grant No. NNX08AR22G issued through the Planetary Science Division of the NASA Science Mission Directorate, the National Science Foundation Grant No. AST-1238877, the University of Maryland, Eotvos Lorand University (ELTE), the Los Alamos National Laboratory, and the Gordon and Betty Moore Foundation.

\section{References}

Abbott, B. P., Abbott, R., Abbott, T. D., et al. 2017, Phys. Rev. Lett., 119, 161101 Abolfathi, B., Aguado, D. S., Aguilar, G., et al. 2018, ApJS, 235, 42 Abrarov, S. M., \& Quine, B. M. 2015a, J. Math. Res., 7, 163 Abrarov, S. M., \& Quine, B. M. 2015b, J. Math. Res., 7, 44 Ackermann, M., Ajello, M., Asano, K., et al. 2014, Science, 343, 42 Amati, L., Guidorzi, C., Frontera, F., et al. 2008, MNRAS, 391, 577 Antonelli, L. A., Maund, J. R., Palazzi, E., et al. 2010, GRB Coordinates Network, Circular Service, No. 10620

Arabsalmani, M., Møller, P., Perley, D. A., et al. 2018, MNRAS, 473, 3312 Arcavi, I., Hosseinzadeh, G., Howell, D. A., et al. 2017, Nature, 551, 64

Ashall, C., Pian, E., Mazzali, P. A., et al. 2017, Nat. Astron., submitted [arXiv:1702.04339].

Astropy Collaboration (Robitaille, T. P., et al.) 2013, A\&A, 558, A33

Axelsson, M., Baldini, L., Barbiellini, G., et al. 2012, ApJ, 757, L31

Bañados, E., Venemans, B. P., Mazzucchelli, C., et al. 2017, Nature, 553, 473

Band, D. L. 2006, ApJ, 644, 378

Barthelmy, S. D. 2000, AIP Conf. Proc., 526, 731

Barthelmy, S. D., Barbier, L. M., Cummings, J. R., et al. 2005, Space Sci. Rev., 120,143

Baumgartner, W. H., Tueller, J., Markwardt, C. B., et al. 2013, ApJS, 207, 19

Benner, D. C., Rinsland, C. P., Devi, V. M., Smith, M. A. H., \& Atkins, D. 1995, JQSRT, 53, 705

Berger, E. 2011, GRB Coordinates Network, Circular Service, No. 12193

Berger, E., Kulkarni, S., \& Frail, D. 2003, ApJ, 590, 379

Berger, E., Chornock, R., Holmes, T. R., et al. 2011, ApJ, 743, 204

Berger, E., Fong, W., \& Chornock, R. 2013, ApJ, 774, L23

Bloom, J. S., Kulkarni, S. R., \& Djorgovski, S. G. 2002, AJ, 123, 1111

Bolmer, J., Ledoux, C., Wiseman, P., et al. 2019, A\&A, 623, A43

Bouwens, R. J., Illingworth, G. D., Oesch, P. A., et al. 2015, ApJ, 803, 1

Boyd, R. W. 1978, J. Opt. Soc. Am., 68, 877

Briggs, M. S., Paciesas, W. S., Pendleton, G. N., et al. 1996, ApJ, 459, 40

Bufano, F., Bufano, F., Benetti, S., et al. 2010, GRB Coordinates Network, Circular Service, No. 10543

Bufano, F., Pian, E., Sollerman, J., et al. 2012, ApJ, 753, 67

Burgess, J. M., Greiner, J., Begue, D., et al. 2017, ArXiv e-prints [arXiv: 1710.05823$]$

Burrows, D. N., Hill, J. E., Nousek, J. A., et al. 2005, Space Sci. Rev., 120, 165
Burrows, D. N., Kennea, J. A., Abbey, A. F., et al. 2007, International Society for Optics and Photonics, 668607

Campana, S., Salvaterra, R., Melandri, A., et al. 2012, MNRAS, 421, 1697

Cano, Z., Bersier, D., Guidorzi, C., et al. 2011, ApJ, 740, 41

Cano, Z., Izzo, L., de Ugarte Postigo, A., et al. 2017, A\&A, 605, A107

Cardelli, J. A., Clayton, G. C., \& Mathis, J. S. 1989, ApJ , 345, 245

Castro-Tirado, A. J., Møller, P., García-Segura, G., et al. 2010, A\&A, 517, A61

Chambers, K. C., Magnier, E. A., Metcalfe, N., et al. 2016, ArXiv e-prints [arXiv:1612.05560]

Chen, T. W., Klose, S., Guelbenzu, A. N., et al. 2016, GRB Coordinates Network, Circular Service, No. 19975

Chornock, R., \& Berger, E. 2011, GRB Coordinates Network, Circular Service, No. 11518

Chornock, R., Berger, E., Kasen, D., et al. 2017, ApJ, 848, L19

Christensen, L., Fynbo, J. P. U., Prochaska, J. X., et al. 2011, ApJ, 727, 73

Christensen, L., Vergani, S. D., Schulze, S., et al. 2017, A\&A, 608, A84

Cobb, B. E., Bloom, J. S., Perley, D. A., et al. 2010, ApJ, 718, L150

Coulter, D. A., Foley, R. J., Kilpatrick, C. D., et al. 2017, Science, 358, 1556

Covino, S., Melandri, A., Salvaterra, R., et al. 2013, MNRAS, 432, 1231

Coward, D. M., Howell, E. J., Branchesi, M., et al. 2013, MNRAS, 432, 2141

Cowperthwaite, P. S., Berger, E., Villar, V. A., et al. 2017, ApJ, 848, L17

Cucchiara, A., Fumagalli, M., Rafelski, M., et al. 2015, ApJ, 804, 51

D'Avanzo, P., Sparre, M., Watson, D., et al. 2011a, GRB Coordinates Network, Circular Service, No. 12284

D’Avanzo, P., Vergani, S. D., Flores, H., et al. 2011b, GRB Coordinates Network, Circular Service, No. 12542

D’Elia, V., \& Stratta, G. 2011, A\&A, 532, A48

D'Elia, V., Fiore, F., Perna, R., et al. 2009, ApJ, 694, 332

D’Elia, V., Fynbo, J. P. U., Covino, S., et al. 2010, A\&A, 523, A36

D'Elia, V., Goldoni, P., Malesani, D., et al. 2012a, GRB Coordinates Network, Circular Service, No. 13227

D’Elia, V., Goldoni, P., Xu, D., et al. 2012b, GRB Coordinates Network, Circular Service, No. 13494

D'Elia, V., Xu, D., de Ugarte Postigo, A., et al. 2012c, GRB Coordinates Network, Circular Service, No. 13507

D’Elia, V., Fynbo, J. P. U., Goldoni, P., et al. 2014, A\&A, 564, A38

D'Elia, V., Krühler, T., Wiersema, K., et al. 2015a, GRB Coordinates Network, Circular Service, No. 18187

D’Elia, V., Krühler, T., Xu, D., et al. 2015b, GRB Coordinates Network, Circular Service, No. 18318

D'Elia, V., Xu, D., Malesani, D., et al. 2016, GRB Coordinates Network, Circular Service, No. 19192

De Pasquale, M., Piro, L., Perna, R., et al. 2003, ApJ, 592, 1018

Dessauges-Zavadsky, M., Chen, H.-W., Prochaska, J. X., Bloom, J. S., \& Barth, A. J. 2006, ApJ, 648, L89

de Ugarte Postigo, A., D’Odorico, S., Vernet, J., et al. 2009a, GRB Coordinates, Network, 9015

de Ugarte Postigo, A., Goldoni, P., Thöne, C. C., et al. 2009b, GRB Coordinates Network, Circular Service, No. 1042

de Ugarte Postigo, A., Goldoni, P., Thöne, C. C., et al. 2010, A\&A, 513, A42

de Ugarte Postigo, A., Thöne, C. C., Goldoni, P., \& Fynbo, J. P. U. 2011a, AN, 332, 297

de Ugarte Postigo, A., Goldoni, P., Milvang-Jensen, B., et al. 2011b, GRB Coordinates Network, Circular Service, No. 11579

de Ugarte Postigo, A., Fynbo, J. P. U., Jakobsson, P., et al. 2011c, GRB Coordinates Network, Circular Service, No. 12258

de Ugarte Postigo, A., Blazek, M., Janout, P., et al. 2014a, in Proc. SPIE, 9152, eds. G. Chiozzi, N. M. Radziwill, et al. , 91520B

de Ugarte Postigo, A., Thöne, C. C., Rowlinson, A., et al. 2014b, A\&A, 563, A62

de Ugarte Postigo, A., Krühler, T., Flores, H., \& Fynbo, J. P. U. 2015a, GRB Coordinates Network, Circular Service, No. 17523

de Ugarte Postigo, A., Xu, D., Malesani, D., \& Tanvir, N. R. 2015b, GRB Coordinates Network, Circular Service, No. 17822

de Ugarte Postigo, A., Thöne, C., Lombardi, G., \& Perez, A. 2015c, GRB Coordinates Network, Circular Service, No. 18274

de Ugarte Postigo, A., Pugliese, G., Xu, D., \& Malesani, D. 2016a, GRB Coordinates Network, Circular Service, No. 18886

de Ugarte Postigo, A., Malesani, D., Krühler, T., et al. 2016b, GRB Coordinates Network, Circular Service, No. 19154

de Ugarte Postigo, A., Kann, D. A., Izzo, L., et al. 2016c, GRB Coordinates Network, Circular Service, No. 20014

de Ugarte Postigo, A., Cano, Z., Izzo, L., et al. 2016d, GRB Coordinates Network, Circular Service, No. 20342

de Ugarte Postigo, A., Thöne, C. C., Bolmer, J., et al. 2018, A\&A, 620, A119

Djorgovski, S. G., Frail, D. A., Kulkarni, S. R., et al. 2001, ApJ, 562, 654

Elliott, J., Schmidl, S., Greiner, J., et al. 2013, GRB Coordinates Network, Circular Service, No. 14898 
Evans, P. A., Beardmore, A. P., Page, K. L., et al. 2007, A\&A, 469, 379 Evans, P. A., Beardmore, A. P., Page, K. L., et al. 2009, MNRAS, 397, 1177 Filippenko, A. V. 1982, PASP, 94, 715

Fiore, F., D'Elia, V., Lazzati, D., et al. 2005, ApJ, 624, 853

Flores, H., Fynbo, J. P. U., de Ugarte Postigo, A., et al. 2010, GRB Coordinates Network, Circular Service, No. 11317

Flores, H., Covino, S., Xu, D., et al. 2013a, GRB Coordinates Network, Circular Service, No. 14491

Flores, H., Covino, S., de Ugarte Postigo, A., et al. 2013b, GRB Coordinates Network, Circular Service, No. 14493

Fong, W., Berger, E., Chornock, R., et al. 2013, ApJ, 769, 56

Freedman, D. L., \& Waxman, E. 2001, ApJ, 547, 922

Freudling, W., Romaniello, M., Bramich, D. M., et al. 2013, A\&A, 559, A96

Friis, M., De Cia, A., Kruhler, T., et al. 2015, MNRAS, 451, 167

Fruchter, A. S., Levan, A. J., Strolger, L., et al. 2006, Nature, 441, 463

Fynbo, J. U., Jensen, B. L., Gorosabel, J., et al. 2001, A\&A, 369, 373

Fynbo, J. P. U., Jakobsson, P., Prochaska, J. X., et al. 2009, ApJS, 185, 175

Fynbo, J. P. U., Laursen, P., Ledoux, C., et al. 2010, MNRAS, 408, 2128

Fynbo, J. P. U., de Ugarte Postigo, A., D’Elia, V., Xu, D., \& Malesani, D. 2012a GRB Coordinates Network, Circular Service, No. 13477

Fynbo, J. P. U., Tanvir, N. R., D'Elia, V., et al. 2012b, GRB Coordinates Network, Circular Service, No. 14120

Fynbo, J. P. U., Xu, D., Malesani, D., et al. 2013, GRB Coordinates Network, Circular Service, No. 14286

Fynbo, J. P. U., Tanvir, N. R., Jakobsson, P., et al. 2014a, GRB Coordinates Network, Circular Service, No. 16217

Fynbo, J. P. U., Krühler, T., Leighly, K., et al. 2014b, A\&A, 572, A12

Galama, T. J., \& Wijers, R. A. M. J. 2001, ApJ, 549, L209

Gehrels, N., White, N., Barthelmy, S., et al. 2004, ApJ, 611, 1005

Gehrels, N., Ramirez-Ruiz, E., \& Fox, D. 2009, ARA\&A, 47, 567

Ghirlanda, G.2007, R. Soc. London Phil. Trans. Ser. A, 365, 1385

Ginsburg, A., Parikh, M., Woillez, J., et al. 2016, Astroquery V0.3.1, DOI 10.5281/zenodo.44961

Goldoni, P., Royer, F., François, P., et al. 2006, Proc. SPIE, 6269, 62692K

Goldoni, P., Flores, H., Malesani, D., et al. 2010, GRB Coordinates Network, Circular Service, No. 10684

Goldoni, P., de Ugarte Postigo, A., \& Fynbo, J. P. U. 2013, GRB Coordinates Network, Circular Service, No. 15571

Goldstein, A., Veres, P., Burns, E., et al. 2017, ApJ, 848, L14

Graff, P. B., Lien, A. Y., Baker, J. G., \& Sakamoto, T. 2016, ApJ, 818, 55

Greiner, J., Bornemann, W., Clemens, C., et al. 2008, PASP, 120, 405

Greiner, J., Krühler, T., Klose, S., et al. 2011, A\&A, 526, A30

Greiner, J., Yu, H.-F., Krühler, T., et al. 2014, A\&A, 568, A75

Greiner, J., Mazzali, P. A., Kann, D. A., et al. 2015, Nature, 523, 189

Greiner, J., Bolmer, J., Wieringa, M., et al. 2018, A\&A, 614, A29

Groot, P. J., Galama, T. J., van Paradijs, J., et al. 1998, ApJ, 493, L27

Groot, P., Kaper, L., Ellerbroek, L., et al. 2010, GRB Coordinates Network Circular Service, No. 10441

Hamuy, M., Suntzeff, N. B., Heathcote, S. R., et al. 1994, PASP, 106, 566

Hartoog, O. E., Malesani, D., Wiersema, K., et al. 2012, GRB Coordinates Network, Circular Service, No. 13730

Hartoog, O. E., Wiersema, K., Vreeswijk, P. M., et al. 2013a, MNRAS, 430, 2739

Hartoog, O. E., Xu, D., Malesani, D., et al. 2013b, GRB Coordinates Network, Circular Service, No. 15494

Hartoog, O. E., Malesani, D., Sanchez-Ramirez, R., et al. 2014, GRB Coordinates Network, Circular Service, No. 16437

Hartoog, O. E., Malesani, D., Fynbo, J. P. U., et al. 2015, A\&A, 580, A139

Heintz, K. E., Malesani, D., de Ugarte Postigo, A., et al. 2016, GRB Coordinates Network, Circular Service, No. 20020

Heintz, K. E., Fynbo, J. P. U., Jakobsson, P., et al. 2017, A\&A, 601, A83

Heintz, K. E., Watson, D., Jakobsson, P., et al. 2018a, MNRAS, 479, 3456

Heintz, K. E., Malesani, D., Wiersema, K., et al. 2018b, MNRAS, 474, 2738

Hjorth, J., \& Bloom, J. S. 2011, in Gamma-Ray Bursts, eds. C. Kouveliotou, R. A. M. J. Wijers, \& S. Woosley (Cambridge: Cambridge University Press) 1973, 169

Hjorth, J., Malesani, D., Jakobsson, P., et al. 2012, ApJ, 756, 187

Hjorth, J., Melandri, A., Malesani, D., Krühler, T., \& Xu, D. 2013, GRB Coordinates Network, Circular Service, No. 14365

Hogg, D. W., \& Fruchter, A. S. 1999, ApJ, 520, 54

Horne, K. 1986, PASP, 98, 609

Hunter, J. D. 2007, Comput. Sci. Eng., 9, 99

Izzo, L., Thöne, C. C., Schulze, S., et al. 2017, MNRAS, 472, 4480

Jakobsson, P., Hjorth, J., Fynbo, J. P. U., et al. 2004, ApJ, 617, L21

Jakobsson, P., Frail, D. A., Fox, D. B., et al. 2005, ApJ, 629, 45

Jakobsson, P., Levan, A., Fynbo, J. P. U., et al. 2006, A\&A, 447, 897

Jakobsson, P., Hjorth, J., Malesani, D., et al. 2012, ApJ, 752, 62

Japelj, J., Covino, S., Gomboc, A., et al. 2015, A\&A, 579, A74
Jones, A., Noll, S., Kausch, W., Szyszka, C., \& Kimeswenger, S. 2013, A\&A, 560, A91

Kann, D. A., Schady, P., Olivares, E. F., et al. 2018, A\&A, 617, A122

Kausch, W., Noll, S., Smette, A., et al. 2015, A\&A, 576, A78

Kilpatrick, C. D., Foley, R. J., Kasen, D., et al. 2017, Science, 358, 1583

Klose, S., Schmidl, S., Kann, D. A., et al. 2019, A\&A, 622, A138

Knust, F., Kann, D. A., Krühler, T., \& Greiner, J. 2015, GRB Coordinates Network, Circular Service, No. 17767

Krühler, T., Greiner, J., Schady, P., et al. 2011, A\&A, 534, A108

Krühler, T., Malesani, D., Milvang-Jensen, B., et al. 2012a, ApJ, 758, 46

Krühler, T., Fynbo, J. P. U., Milvang-Jensen, B., Tanvir, N., \& Jakobsson, P. 2012b, GRB Coordinates Network, Circular Service, No. 13134

Krühler, T., Tanvir, N. R., de Ugarte Postigo, A., et al. 2012c, GRB Coordinates Network, Circular Service, No. 13930

Krühler, T., Ledoux, C., Fynbo, J. P. U., et al. 2013a, A\&A, 557, A18

Krühler, T., Malesani, D., Xu, D., et al. 2013b, GRB Coordinates Network, Circular Service, No. 14264

Krühler, T., Xu, D., Sanchez-Ramirez, R., et al. 2013c, GRB Coordinates Network, Circular Service, No. 14390

Krühler, T., Tanvir, N. R., Malesani, D., Xu, D., \& Fynbo, J. P. U. 2014a, GRB Coordinates Network, Circular Service, No. 15900

Krühler, T., Malesani, D., de Ugarte Postigo, A., Melandri, A., \& Fynbo, J. P. U. 2014b, GRB Coordinates Network, Circular Service, No. 16194

Krühler, T., Vreeswijk, P. M., \& Fynbo, J. P. U. 2014c, GRB Coordinates Network, Circular Service, No. 16401

Krühler, T., Malesani, D., Fynbo, J. P. U., et al. 2015a, A\&A, 581, A125

Krühler, T., Xu, D., Fynbo, J. P. U., et al. 2015b, GRB Coordinates Network, Circular Service, No. 17420

Krühler, T., Malesani, D., Xu, D., et al. 2016a, GRB Coordinates Network, Circular Service, No. 19186

Krühler, T., Xu, D., Bolmer, J., et al. 2016b, GRB Coordinates Network, Circular Service, No. 19971

Letchworth, K. L., \& Benner, D. C. 2007, JQSRT, 107, 173

Levan, A. J., Tanvir, N. R., Starling, R. L. C., et al. 2013, ApJ, 781, 13

Levesque, E. M., Kewley, L. J., Graham, J. F., \& Fruchter, A. S. 2010, ApJ, 712, L26

Lien, A., Sakamoto, T., Gehrels, N., et al. 2014, ApJ, 783, 24

Lien, A., Sakamoto, T., Barthelmy, S. D., et al. 2016, ApJ, 829, 7

LIGO Scientific Collaboration, et al. 2017, ApJ, 848, L12

Lipunov, V. M., Gorbovskoy, E., Kornilov, V. G., et al. 2017, ApJ, 850, L1

Littlejohns, O. M., Tanvir, N. R., Willingale, R., et al. 2013, MNRAS, 436, 3640

López, S., D’Odorico, V., Ellison, S. L., et al. 2016, A\&A, 594, A91

Lyman, J. D., Levan, A. J., Tanvir, N. R., et al. 2017, MNRAS, 1817, 220

Malesani, D., \& Palazzi, E. 2010, GRB Coordinates Network, Circular Service, No. 10631

Malesani, D., Fynbo, J. P. U., Christensen, L., et al. 2009a, GRB Coordinates, Network, 9761

Malesani, D., Goldoni, P., Fynbo, J. P. U., et al. 2009b, GRB Coordinates, Network, 9942

Malesani, D., Schulze, S., de Ugarte Postigo, A., et al. 2012, GRB Coordinates Network, Circular Service, No. 13649

Malesani, D., Xu, D., Fynbo, J. P. U., et al. 2013a, GRB Coordinates Network, Circular Service, No. 14291

Malesani, D., Krühler, T., Perley, D., et al. 2013b, GRB Coordinates Network, Circular Service, No. 14225

Malesani, D., Krühler, T., Xu, D., et al. 2015a, GRB Coordinates Network, Circular Service, No. 17755

Malesani, D., Tanvir, N. R., Krühler, T., et al. 2015b, GRB Coordinates Network, Circular Service, No. 18540

Malesani, D., Krühler, T., Heintz, K. E., \& Fynbo, J. P. U. 2016, GRB Coordinates Network, Circular Service, No. 20180

Markwardt, C. B., Barthelmy, S. D., Cummings, J. R., et al. 2016, GRB Coordinates Network, Circular Service, No. 19974

Maselli, A., Melandri, A., Nava, L., et al. 2014, Science, 343, 48

Meegan, C. A., Fishman, G. J., Wilson, R. B., et al. 1992, Nature, 355, 143

Melandri, A., Sbarufatti, B., D'Avanzo, P., et al. 2012, MNRAS, 421, 1265

Melandri, A., Bernardini, M. G., D’Avanzo, P., et al. 2015, A\&A, 581, A86

Michałowski, M. J., Xu, D., Stevens, J., et al. 2018, A\&A, 616, A169

Micol, A., Arnaboldi, M., Delmotte, N. A. R., Mascetti, L., \& Retzlaff, J. 2016, Proc. SPIE, 9910, 991030

Milvang-Jensen, B., Goldoni, P., Tanvir, N. R., et al. 2010, GRB Coordinates Network, Circular Service, No. 10876

Modigliani, A., Goldoni, P., Royer, F., et al. 2010, Proc. SPIE, 7737, 773728

Moffat, A. F. J. 1969, A\&A, 3, 455

Molinari, E., Vergani, S. D., Malesani, D., et al. 2007, A\&A, 469, L13

Monet, D. G., Levine, S. E., Canzian, B., et al. 2003, AJ, 125, 984

Morgan, A. N., Perley, D. A., Cenko, S. B., et al. 2014, MNRAS, 440, 1810

Nicholl, M., Berger, E., Kasen, D., et al. 2017, ApJ, 848, L18 
Noll, S., Kausch, W., Barden, M., et al. 2012, A\&A, 543, A92

Noterdaeme, P., Petitjean, P., Carithers, W. C., et al. 2012, A\&A, 547, L1

Nysewander, M., Fruchter, A. S., \& Pe'er, A. 2009, ApJ, 701, 824

Oesch, P. A., Brammer, G., van Dokkum, P. G., et al. 2016, ApJ, 819, 129

Oliphant, T. E. 2007, Comput. Sci. Eng., 9, 10

Olivares, E. F., Greiner, J., Schady, P., et al. 2012, A\&A, 539, A76

Olivares, E. F., Greiner, J., Schady, P., et al. 2015, A\&A, 577, A44

Osborne, J. P., Beardmore, A. P., Evans, P. A., \& Goad, M. R. 2016, GRB Coordinates Network, Circular Service, No. 19969

Pagnini, G., \& Mainardi, F. 2010, JCoAM, 233, 1590

Palmerio, J., Krühler, T., Malesani, D., \& Fynbo, J. P. U. 2017, GRB Coordinates Network, Circular Service, No. 20589

Perley, D. A., Li, W., Chornock, R., et al. 2008, ApJ, 688, 470

Perley, D. A., Cenko, S. B., Bloom, J. S., et al. 2009, AJ, 138, 1690

Perley, D. A., Bloom, J. S., Cenko, S. B., et al. 2010, GRB Coordinates Network, Circular Service, No. 10399

Perley, D. A., Morgan, A. N., Updike, A., et al. 2011, AJ, 141, 36

Perley, D. A., Levan, A. J., Tanvir, N. R., et al. 2013, ApJ, 778, 128

Perley, D. A., Cenko, S. B., Corsi, A., et al. 2014, ApJ, 781, 37

Perley, D. A., Perley, R. A., Hjorth, J., et al. 2015, ApJ, 801, 102

Perley, D. A., Krühler, T., Schulze, S., et al. 2016a, ApJ, 817, 7

Perley, D. A., Tanvir, N. R., Hjorth, J., et al. 2016b, ApJ, 817, 8

Perley, D. A., Hjorth, J., Tanvir, N. R., \& Perley, R. A. 2017a, MNRAS, 465 970

Perley, D. A., Krühler, T., Schady, P., et al. 2017b, MNRAS, 465, L89

Pescalli, A., Ghirlanda, G., Salvaterra, R., et al. 2015, A\&A, 587, A40

Pian, E., D’ Avanzo, P., Benetti, S., et al. 2017, Nature, 551, 67

Piran, T. 2005, Rev. Mod. Phys., 76, 1143

Piranomonte, S., Vergani, S. D., Malesani, D., et al. 2011, GRB Coordinates Network, Circular Service, No. 12164

Planck Collaboration XIII. 2016, A\&A, 594, A13

Pozanenko, A., Mazaeva, E., Sergeev, A., et al. 2015, GRB Coordinates Network, Circular Service, No. 17903

Prochaska, J. X., Chen, H.-W., \& Bloom, J. S. 2006, ApJ, 648, 95

Prochaska, J. X., Chen, H.-W., Dessauges-Zavadsky, M., \& Bloom, J. S. 2007, ApJ, 666, 267

Prochaska, J. X., Chen, H.-W., Wolfe, A. M., Dessauges-Zavadsky, M., \& Bloom, J. S. 2008, ApJ, 672, 59

Prochaska, J. X., Sheffer, Y., Perley, D. A., et al. 2009, ApJ, 691, L27

Pugliese, V., Xu, D., Tanvir, N. R., et al. 2015, GRB Coordinates Network, Circular Service, No. 17672

Pugliese, G., Covino, S., Krühler, T., Xu, D., \& Tanvir, N. R. 2016, GRB Coordinates Network, Circular Service, No. 18982

Rau, A., Krühler, T., \& Greiner, J. 2013, GRB Coordinates Network, Circular Service, No. 15330

Ricker, G. R., \& Team, H. S. 2004, Bull. Am. Astron. Soc., 36, 942

Rol, E., Wijers, R. A. M. J., Kouveliotou, C., Kaper, L., \& Kaneko, Y. 2005, ApJ, 624, 868

Roming, P. W., Kennedy, T. E., Mason, K. O., et al. 2005, Space Sci. Rev., 120, 95

Rossi, A., Klose, S., Ferrero, P., et al. 2012, A\&A, 545, A77

Rossi, A., Piranomonte, S., Savaglio, S., et al. 2014, A\&A, 572, A47

Rowlinson, A., Patruno, A., \& O'Brien, P. T. 2017, MNRAS, 472, 1152

Sakamoto, T., Barthelmy, S. D., Cummings, J. R., et al. 2016, GRB Coordinates Network, Circular Service, No. 19276

Salvaterra, R., Valle, M. D., Campana, S., et al. 2009, Nature, 461, 1258

Salvaterra, R., Campana, S., Vergani, S. D., et al. 2012, ApJ, 749, 68

Sanchez-Ramirez, R., de Ugarte Postigo, A., Krühler, T., et al. 2012, GRB Coordinates Network, Circular Service, No. 14035

Sánchez-Ramírez, R., Hancock, P. J., Jóhannesson, G., et al. 2017, MNRAS, 464, 4624

Sarazin, M., \& Roddier, F. 1990, A\&A, 227, 294

Savaglio, S. 2006, New J. Phys., 8, 195

Savaglio, S., Fall, S. M., \& Fiore, F. 2003, ApJ, 585, 638

Savchenko, V., Ferrigno, C., Kuulkers, E., et al. 2017, ApJ, 848, L15

Schady, P., Krühler, T., Greiner, J., et al. 2015, A\&A, 579, A126

Schlafly, E. F., \& Finkbeiner, D. P. 2011, ApJ, 737, 103

Schlegel, D. J., Finkbeiner, D. P., \& Davis, M. 1998, ApJ, 500, 525

Schulze, S., Covino, S., Flores, H., et al. 2011, GRB Coordinates Network, Circular Service, No. 12770

Schulze, S., Levan, A. J., \& Malesani, D. 2012, GRB Coordinates Network, Circular Service, No. 13257

Schulze, S., Malesani, D., Cucchiara, A., et al. 2014a, A\&A, 566, A102

Schulze, S., Wiersema, K., Xu, D., \& Fynbo, J. P. U. 2014b, GRB Coordinates Network, Circular Service, No. 15831

Schulze, S., Chapman, R., Hjorth, J., et al. 2015, ApJ, 808, 73

Selsing, J., Fynbo, J. P. U., Christensen, L., \& Krogager, J.-K. 2015, A\&A, 585, A87
Selsing, J., Vreeswijk, P. M., Japelj, J., et al. 2016a, GRB Coordinates Network, Circular Service, No. 19274

Selsing, J., Heintz, K. E., Malesani, D., et al. 2016b, GRB Coordinates Network, Circular Service, No. 20061

Selsing, J., Krühler, T., Malesani, D., et al. 2018, A\&A, 616, A48

Sheffer, Y., Prochaska, J. X., Draine, B. T., Perley, D. A., \& Bloom, J. S. 2009, ApJ, 701, L63

Smartt, S. J., Chen, T. W., Jerkstrand, A., et al. 2017, Nature, 551, 75

Smette, A., Sana, H., Noll, S., et al. 2015, A\&A, 576, A77

Soares-Santos, M., Holz, D. E., Annis, J., et al. 2017, ApJ, 848, L16

Sparre, M., de Ugarte Postigo, A., Fynbo, J. P. U., et al. 2011, GRB Coordinates Network, Circular Service, No. 11607

Sparre, M., Sollerman, J., Fynbo, J. P. U., et al. 2011, ApJ, 735, L24

Sparre, M., Hartoog, O. E., Krühler, T., et al. 2014, ApJ, 785, 150

Starling, R. L. C., Wiersema, K., Levan, A. J., et al. 2011, MNRAS, 411, 2792

Sudilovsky, V., Kann, D. A., Schady, P., et al. 2013, GRB Coordinates Network, Circular Service, No. 15250

Tanvir, N. R., Fox, D. B., Levan, A. J., et al. 2009, Nature, 461, 1254

Tanvir, N. R., Vergani, S., Hjorth, J., et al. 2010, GRB Coordinates Network, Circular Service, No. 11123

Tanvir, N. R., Fynbo, J. P. U., Melandri, A., et al. 2012, GRB Coordinates Network, Circular Service, No. 13890

Tanvir, N. R., Levan, A. J., Fruchter, A. S., et al. 2013a, Nature, 500, 547

Tanvir, N. R., Wiersema, K., Xu, D., \& Fynbo, J. P. U. 2013b, GRB Coordinates Network, Circular Service, No. 14882

Tanvir, N. R., Xu, D., Zafar, T., Covino, S., \& Schulze, S. 2015a, GRB Coordinates Network, Circular Service, No. 18080

Tanvir, N. R., Krühler, T., Malesani, D., et al. 2015b, GRB Coordinates Network, Circular Service, No. 18524

Tanvir, N. R., Xu, D., Krühler, T., et al. 2016a, GRB Coordinates Network, Circular Service, No. 19350

Tanvir, N. R., Krühler, T., De Cia, A., et al. 2016b, GRB Coordinates Network, Circular Service, No. 20104

Tanvir, N. R., Krühler, T., Wiersema, K., et al. 2016c, GRB Coordinates Network, Circular Service, No. 20321

Tanvir, N. R., Levan, A. J., González-Fernández, C., et al. 2017, ApJ, 848, L27

Tanvir, N. R., Laskar, T., Levan, A. J., et al. 2018, ApJ, 865, 107

Tanvir, N. R., Fynbo, J. P. U., de Ugarte Postigo, A., et al. 2019, MNRAS, 483, 5380

Tepper García, T. 2006, MNRAS, 369, 2025

Thöne, C. C., Greiner, J., Savaglio, S., \& Jehin, E. 2007, ApJ, 671, 628

Thöne, C. C., Goldoni, P., Covino, S., et al. 2009, GRB Coordinates Network, Circular Service, No. 10233

Thöne, C. C., de Ugarte Postigo, A., Vreeswijk, P., et al. 2010, GRB Coordinates Network, Circular Service, No. 10971

Thöne, C. C., Fynbo, J. P. U., Goldoni, P., et al. 2013, MNRAS, 428, 3590

Troja, E., Lipunov, V. M., Mundell, C. G., et al. 2017a, Nature, 547, 425

Troja, E., Piro, L., van Eerten, H., et al. 2017b, Nature, 551, 71

Trujillo, I., Aguerri, J. A. L., Cepa, J., \& Gutiérrez, C. M. 2001, MNRAS, 328, 977

Ukwatta, T. N., \& Wózniak, P. R. 2016, MNRAS, 455, 703

Ukwatta, T. N., Barthelmy, S. D., Beardmore, A. P., et al. 2016, GRB Coordinates Network, Circular Service, No. 19148

van der Horst, A. J., Kouveliotou, C., Gehrels, N., et al. 2009, ApJ, 699, 1087

Van Der Walt, S., Colbert, S. C., \& Varoquaux, G. 2011, Comput. Sci. Eng., 13, 22

van Dokkum, P. G. 2001, PASP, 113, 1420

van Paradijs, J., Kouveliotou, C., \& Wijers, R. A. M. J. 2000, ARA\&A, 38, 379

Vergani, S. D., Petitjean, P., Ledoux, C., et al. 2009, A\&A, 503, 771

Vergani, S. D., D’Avanzo, P., Malesani, D., et al. 2010a, GRB Coordinates Network, Circular Service, No. 10495

Vergani, S. D., D’Avanzo, P., Levan, A. J., et al. 2010b, GRB Coordinates Network, Circular Service, No. 10512

Vergani, S. D., Levan, A. J., D’Avanzo, P., et al. 2010c, GRB Coordinates Network, Circular Service, No. 10513

Vergani, S. D., Flores, H., Covino, S., et al. 2011a, A\&A, 535, A127

Vergani, S. D., Piranomonte, S., Hartoog, O. E., et al. 2011b, GRB Coordinates Network, Circular Service, No. 12677

Vergani, S. D., Salvaterra, R., Japelj, J., et al. 2015, A\&A, 581, A102

Vernet, J., Kerber, F., Mainieri, V., et al. 2009, Proc. IAU, 5, 535

Vernet, J., Dekker, H., D'Odorico, S., et al. 2011, A\&A, 536, A105

Vreeswijk, P. M., Ledoux, C., Smette, A., et al. 2007, A\&A, 468, 83

Vreeswijk, P. M., Kaufer, A., Spyromilio, J., et al. 2010, Proc. SPIE, 7737, $77370 \mathrm{M}$

Vreeswijk, P., Fynbo, J., \& Melandri, A. 2011, GRB Coordinates Network, Circular Service, No. 12648 
Vreeswijk, P. M., Ledoux, C., Raassen, A. J. J., et al. 2013, A\&A, 549, A22

Watson, D., \& Jakobsson, P. 2012, ApJ, 754, 89

Watson, D., Zafar, T., Andersen, A. C., et al. 2013, ApJ, 768, 23

Wiersema, K., Flores, H., D'Elia, V., et al. 2011, GRB Coordinates Network, Circular Service, No. 12431

Wiersema, K., Curran, P. A., Krühler, T., et al. 2012a, MNRAS, 426, 2

Wiersema, K., Goldoni, P., Fynbo, J. P. U., et al. 2012b, GRB Coordinates Network, Circular Service, No. 12991

Wijers, R. A. M. J., Bloom, J. S., Bagla, J. S., \& Natarajan, P. 1998, MNRAS, 294, L13

Wright, E. L., Eisenhardt, P. R. M., Mainzer, A. K., et al. 2010, AJ, 140, 1868

Xu, D., Fynbo, J. P. U., D’Elia, V., \& Tanvir, N. R. 2012, GRB Coordinates Network, Circular Service, No. 13460

Xu, D., Malesani, D., Krühler, T., et al. 2013a, GRB Coordinates Network, Circular Service, No. 14273

Xu, D., De Ugarte Postigo, A., Leloudas, G., et al. 2013b, ApJ , 776, 98

Xu, D., de Ugarte Postigo, A., Malesani, D., et al. 2013c, GRB Coordinates Network, Circular Service, No. 14757

Xu, D., Malesani, D., Schulze, S., et al. 2013d, GRB Coordinates Network, Circular Service, No. 14816

Xu, D., de Ugarte Postigo, A., Malesani, D., et al. 2013e, GRB Coordinates Network, Circular Service, No. 14956

Xu, D., Malesani, D., Schulze, S., et al. 2013f, GRB Coordinates Network, Circular Service, No. 15451

Xu, D., Malesani, D., Tanvir, N., Krühler, T., \& Fynbo, J. 2013g, GRB Coordinates Network, Circular Service, No. 15450

Xu, D., Malesani, D., Tanvir, N. R., et al. 2014a, GRB Coordinates Network, Circular Service, No. 15645

Xu, D., Levan, A. J., Fynbo, J. P. U., et al. 2014b, GRB Coordinates Network, Circular Service, No. 16983

Xu, D., Vreeswijk, P. M., Fynbo, J. P. U., et al. 2014c, GRB Coordinates Network, Circular Service, No. 17040

Xu, D., Levan, A. J., de Ugarte Postigo, A., et al. 2015a, GRB Coordinates Network, Circular Service, No. 17832

Xu, D., Tanvir, N. R., Malesani, D., \& Fynbo, J. P. U. 2015b, GRB Coordinates Network, Circular Service, No. 18506

Xu, D., Malesani, D., Fynbo, J. P. U., et al. 2016a, GRB Coordinates Network, Circular Service, No. 19600

Xu, D., Heintz, K. E., Malesani, D., Wiersema, K., \& Fynbo, J. P. U. 2016b, GRB Coordinates Network, Circular Service, No. 19773

Xu, D., Heintz, K. E., Malesani, D., \& Fynbo, J. P. U. 2017, GRB Coordinates Network, Circular Service, No. 20458

Zafar, T., Møller, P., Watson, D., et al. 2018a, MNRAS, 480, 108

Zafar, T., Watson, D., Møller, P., et al. 2018b, MNRAS, 479, 1542

Zheng, W., Filippenko, A. V., Yuk, H., Zhu, Y., \& Perley, D. A. 2015, GRB Coordinates Network, Circular Service, No. 18273

Zitrin, A., Labbé, I., Belli, S., et al. 2015, ApJ, 810, L12

${ }^{1}$ Dark Cosmology Centre, Niels Bohr Institute, University of Copenhagen, Juliane Maries Vej 30, 2100 København Ø, Denmark e-mail: jselsing@dark-cosmology.dk

2 The Cosmic Dawn Center (DAWN), Niels Bohr Institute, University of Copenhagen, Juliane Maries Vej 30, 2100 Copenhagen $\varnothing$, Denmark

3 DTU-Space, Technical University of Denmark, Elektrovej 327, 2800 Kgs. Lyngby, Denmark

${ }^{4}$ DTU Space, National Space Institute, Technical University of Denmark, Elektrovej 327, 2800 Lyngby, Denmark

5 APC, Astroparticule et Cosmologie, Université Paris Diderot, CNRS/IN2P3, CEA/Irfu, Observatoire de Paris, Sorbonne Paris
Cité, 10, rue Alice Domon et Léonie Duquet, 75205 Paris Cedex 13, France

${ }^{6}$ Max-Planck Institut für extraterrestrische Physik, Giessenbachstraße 1, 85748 Garching, Germany

7 INAF - Osservatorio Astronomico di Roma, Via Frascati 33, 00078 Monte Porzio Catone (Roma), Italy

${ }^{8}$ IRFU, CEA, Université Paris-Saclay, 91191 Gif-sur-Yvette, France

9 Université Paris Diderot, AIM, Sorbonne Paris Cité, CEA, CNRS, 91191 Gif-sur-Yvette, France

10 European Southern Observatory, Alonso de Córdova 3107, Vitacura, Casilla 19001, Santiago 19, Chile

11 Instituto de Astrofísica de Andalucía (IAA-CSIC), Glorieta de la Astronomía s/n, 18008 Granada, Spain

12 INAF - Osservatorio Astronomico di Brera, Via Bianchi 46, 23807 Merate (LC), Italy

13 Space Science Data Center - Agenzia Spaziale Italiana, Via del Politecnico, s.n.c., 00133 Roma, Italy

14 European Southern Observatory, Karl-Schwarzschild Str. 2, 85748 Garching bei München, Germany

15 GEPI, Observatoire de Paris, PSL University, CNRS, 5 Place Jules Janssen, 92195 Meudon, France

16 KTH Royal Institute of Technology, Department of Physics, 10691 Stockholm, Sweden

17 The Oskar Klein Centre for Cosmoparticle Physics, AlbaNova University Centre, 10691 Stockholm, Sweden

18 Center for Astrophysics and Cosmology, University of Nova Gorica, Vipavska 11c, 5270 Ajdovščina, Slovenia

19 Department of Astrophysics, IMAPP, Radboud University Nijmegen, PO Box 9010, 6500 GL Nijmegen, The Netherlands

20 Anton Pannekoek Institute for Astronomy, University of Amsterdam, Science Park 904, 1098 XH Amsterdam, The Netherlands

21 Centre for Astrophysics and Cosmology, Science Institute, University of Iceland, Dunhagi 5, 107 Reykjavik, Iceland

22 Department of Physics, University of Warwick, Coventry CV4 7AL, UK

23 IASF/INAF Bologna, Via Piero Gobetti 101, 40129 Bologna, Italy

${ }^{24}$ Sorbonne Université, CNRS, UMR7095, Institut d'Astrophysique de Paris, 75014 Paris, France

25 Astrophysics Research Institute, Liverpool John Moores University, IC2, Liverpool Science Park, 146 Brownlow Hill, Liverpool L3 5RF, UK

26 INAF, Istituto Astrofisica e Planetologia Spaziali, Via Fosso del Cavaliere 100, 00133 Roma, Italy

27 Physics Department, University of Calabria, 87036 Arcavacata di Rende, Italy

28 Department of Particle Physics and Astrophysics, Weizmann Institute of Science, 234 Herzl Street, Rehovot 761000, Israel

29 Department of Astronomy, Stockholm University, AlbaNova, 10691 Stockholm, Sweden

30 Institut für Physik und Astronomie, Universität Potsdam, KarlLiebknecht-Str. 24/25, 14476, Golm, Germany

31 Department of Physics and Astronomy, University of Leicester, University Road, Leicester LE1 7RH, UK

32 CAS Key Laboratory of Space Astronomy and Technology, National Astronomical Observatories, Chinese Academy of Sciences, Beijing 100012, PR China

33 Australian Astronomical Observatory, PO Box 915, North Ryde, NSW 1670, Australia 
Appendix A: Additional material

Table A.1. Full sample of afterglows and hosts observed in the program.

\begin{tabular}{|c|c|c|c|c|c|c|c|c|c|}
\hline GRB & Obs date & $\begin{array}{c}\text { Exptime } \\
(\mathrm{ks})\end{array}$ & $\begin{array}{l}\text { Slit width } \\
(\operatorname{arcsec})\end{array}$ & Airmass & $\begin{array}{c}\text { Seeing } \\
(\operatorname{arcsec})\end{array}$ & $\begin{array}{l}\Delta t \\
(\mathrm{~h})\end{array}$ & $\operatorname{Mag}_{\mathrm{acq}}$ & Redshift & Notes \\
\hline GRB090313 ${ }^{a}$ & 2009-03-15 & $6.9 / 6.9 / 6.9$ & $1.0 / 0.9 / 0.9$ & $1.2-1.4$ & 1.5 & 45 & 21.6 & 3.374 & C.1 \\
\hline GRB090530 & $2009-05-30$ & $4.8 / 4.8 / 4.8$ & $1.0 / 1.2 / 1.2$ & $1.6-2.2$ & 1.7 & 20.6 & 22 & 1.266 & C. 2 \\
\hline GRB090809 $^{a}$ & $2009-08-10$ & $7.2 / 7.2 / 7.2$ & $1.0 / 0.9 / 0.9$ & $1.2-1.1$ & 1.1 & 10.2 & 21 & 2.737 & C. 3 \\
\hline GRB090926A $^{a}$ & $2009-09-27$ & 7.2/7.2/7.2 & $1.0 / 0.9 / 0.9$ & $1.4-1.5$ & 0.7 & 22 & 17.9 & 2.106 & C. 4 \\
\hline GRB091018 & 2009-10-18 & $2.4 / 2.4 / 2.4$ & $1.0 / 0.9 / 0.9$ & $2.1-1.8$ & 1.0 & 3.5 & 19.1 & 0.971 & C.5 \\
\hline GRB091127 & 2009-12-02 & $6.0 / 6.0 / 6.0$ & $1.0 / 0.9 / 0.9$ & $1.1-1.2$ & 1.0 & 101 & 21.2 & 0.490 & C.6 \\
\hline GRB100205A & $2010-02-08$ & $10.8 / 10.8 / 10.8$ & $1.0 / 0.9 / 0.9$ & $1.9-1.8$ & 0.9 & 71 & $>24$ & - & C.7 \\
\hline GRB100219A & $2010-02-20$ & $4.8 / 4.8 / 4.8$ & $1.0 / 0.9 / 0.9$ & $1.3-1.1$ & 0.8 & 12.5 & 23 & 4.667 & C. 8 \\
\hline GRB100316B & $2010-03-16$ & $2.4 / 2.4 / 2.4$ & $1.0 / 0.9 / 0.9$ & $2.0-2.4$ & 0.6 & 0.7 & 18.2 & 1.180 & C.9 \\
\hline GRB100316D-1 ${ }^{b}$ & 2010-03-17 & $3.6 / 3.6 / 3.6$ & $1.0 / 0.9 / 0.9$ & $1.2-1.3$ & 0.8 & 10 & 21.5 & 0.059 & C. 10 \\
\hline GRB100316D-2 & 2010-03-19 & $2.4 / 2.4 / 2.4$ & $1.0 / 0.9 / 0.9$ & $1.1-1.2$ & 0.9 & 58 & 20.2 & 0.059 & C. 10 \\
\hline GRB100316D-3 & $2010-03-20$ & $2.6 / 2.6 / 3.2$ & $1.0 / 0.9 / 0.9$ & $1.1-1.2$ & 1.1 & 79 & 19.9 & 0.059 & C. 10 \\
\hline GRB100316D-4 & 2010-03-21 & $2.6 / 2.6 / 3.2$ & $1.0 / 0.9 / 0.9$ & $1.1-1.2$ & 1.5 & 101 & 19.9 & 0.059 & C. 10 \\
\hline GRB100418A-1 & 2010-04-19 & $4.8 / 4.8 / 4.8$ & $1.0 / 0.9 / 0.9$ & $1.6-1.3$ & 0.7 & 8.4 & 18.1 & 0.624 & C. 11 \\
\hline GRB100418A-2 & 2010-04-20 & $4.8 / 4.8 / 4.8$ & $1.0 / 0.9 / 0.9$ & $1.2-1.3$ & 0.6 & 34 & 19.2 & 0.624 & C. 11 \\
\hline GRB100418A-3 & 2010-04-21 & $4.8 / 4.8 / 4.8$ & $1.0 / 0.9 / 0.9$ & $1.2-1.4$ & 0.7 & 58 & $>24$ & 0.624 & C. 11 \\
\hline GRB100424A ${ }^{c}$ & 2013-03-11 & $4.8 / 4.8 / 4.8$ & $1.0 / 0.9 / 0.9$ & $1.1-1.2$ & 0.9 & 25239 & $>24$ & 2.465 & C. 12 \\
\hline GRB100425A & $2010-04-25$ & $2.4 / 2.4 / 2.4$ & $1.0 / 0.9 / 0.9$ & $1.5-1.3$ & 0.7 & 4 & 20.6 & 1.755 & C. 13 \\
\hline $\mathrm{GRB} 100615 \mathrm{~A}^{c}$ & 2013-03-05 & $4.8 / 4.8 / 4.8$ & $1.0 / 0.9 / 0.9$ & $1.0-1.1$ & 0.9 & 23859 & $>24$ & 1.398 & C. 14 \\
\hline GRB100621A & 2010-06-21 & $2.4 / 2.4 / 2.4$ & $1.0 / 0.9 / 0.9$ & $1.3-1.4$ & 1.0 & 7.1 & 22 & 0.542 & C. 15 \\
\hline GRB100625A $\mathrm{A}^{c, f}$ & 2010-07-07 & $4.8 / 4.8 / 4.8$ & $1.0 / 0.9 / 0.9$ & $1.1-1.0$ & 0.8 & 278.7 & $>24$ & 0.452 & C. 16 \\
\hline GRB100724A ${ }^{a, d}$ & 2010-07-24 & $4.2 / 4.2 / 4.2$ & $1.0 / 0.9 / 0.9$ & $1.5-2.3$ & 0.7 & 0.2 & 19.52 & 1.288 & C. 17 \\
\hline GRB100728B ${ }^{e}$ & $2010-07-29$ & $7.2 / 7.2 / 7.2$ & $1.0 / 0.9 / 0.9$ & $1.5-1.1$ & 0.6 & 22 & 23 & 2.106 & C. 18 \\
\hline GRB100814A-1 ${ }^{d}$ & 2010-08-14 & $0.9 / 0.9 / 0.9$ & $1.0 / 0.9 / 0.9$ & $1.9-1.7$ & 0.5 & 0.9 & 19 & 1.439 & C. 19 \\
\hline GRB100814A-2 & 2010-08-14 & $4.8 / 4.8 / 4.8$ & $1.0 / 0.9 / 0.9$ & $1.5-1.2$ & 0.7 & 2.1 & 19 & 1.439 & C. 19 \\
\hline GRB100814A-3 & $2010-08-18$ & $4.8 / 4.8 / 4.8$ & $1.0 / 0.9 / 0.9$ & $1.2-1.0$ & 0.6 & 98 & 20 & 1.439 & C. 19 \\
\hline $\mathrm{GRB}_{100816 \mathrm{~A}^{f}}$ & 2010-08-17 & $4.8 / 4.8 / 4.8$ & $1.0 / 0.9 / 0.9$ & $1.8-1.6$ & 0.8 & 28.4 & 21.6 & 0.805 & C. 20 \\
\hline GRB100901A & 2010-09-04 & $2.4 / 2.4 / 2.4$ & $1.0 / 0.9 / 0.9$ & $1.5-1.5$ & 1.9 & 66 & $>24$ & 1.408 & C. 21 \\
\hline GRB101219A & 2010-12-19 & 7.2/7.2/7.2 & $1.0 / 0.9 / 0.9$ & $1.1-1.7$ & 1.8 & 3.7 & $>24$ & 0.718 & C. 22 \\
\hline GRB101219B-1 $^{a}$ & $2010-12-20$ & $4.8 / 4.8 / 4.8$ & $1.0 / 0.9 / 0.9$ & $1.6-2.6$ & 1.4 & 11.6 & 20 & 0.552 & C. 23 \\
\hline GRB101219B-2 ${ }^{a}$ & 2011-01-05 & 7.2/7.2/7.2 & $1.0 / 0.9 / 0.9$ & $1.2-2.0$ & 1.0 & 394 & 22.7 & 0.552 & C. 23 \\
\hline $\mathrm{GRB}^{101219 B-3^{a}}$ & $2011-01-25$ & 7.2/7.2/7.2 & $1.0 / 0.9 / 0.9$ & $1.4-2.1$ & 0.7 & 886 & $>24$ & 0.552 & C. 23 \\
\hline GRB110128A & 2011-01-28 & 7.2/7.2/7.2 & $1.0 / 0.9 / 0.9$ & $2.0-1.6$ & 0.6 & 5.5 & 22.5 & 2.339 & C. 24 \\
\hline GRB110407A & 2011-04-08 & $9.6 / 9.6 / 9.6$ & $1.0 / 0.9 / 0.9$ & $1.4-1.3$ & 2.1 & 12.4 & 23 & - & C. 25 \\
\hline $\mathrm{GRB}^{110709 B^{c}}$ & 2013-03-19 & 7.2/7.2/7.2 & $1.0 / 0.9 / 0.9$ & $1.6-1.1$ & 0.9 & 14835 & $>24$ & 2.109 & C. 26 \\
\hline GRB110715A ${ }^{a}$ & 2011-07-16 & $0.6 / 0.6 / 0.6$ & $1.0 / 0.9 / 0.9$ & $1.1-1.1$ & 1.6 & 12.3 & 18.5 & 0.823 & C. 27 \\
\hline GRB110721 $\mathrm{A}^{a}$ & $2011-07-22$ & $2.4 / 2.4 / 2.4$ & $1.0 / 0.9 / 0.9$ & $1.2-1.4$ & 2.3 & 28.7 & $>24$ & 0.382 & C. 28 \\
\hline GRB110808A & 2011-08-08 & $2.4 / 2.4 / 2.4$ & $1.0 / 0.9 / 0.9$ & $1.2-1.1$ & 1.0 & 3.0 & 21.2 & 1.349 & C. 29 \\
\hline GRB110818A & 2011-08-19 & $4.8 / 4.8 / 4.8$ & $1.0 / 0.9 / 0.9$ & $1.3-1.3$ & 0.9 & 6.2 & 22.3 & 3.36 & C. 30 \\
\hline GRB111005A $\mathrm{A}^{a, c}$ & 2013-04-01 & $1.2 / 1.2 / 1.2$ & $1.0 / 0.9 / 0.9$ & $1.3-1.3$ & 0.7 & 13052 & $>24$ & 0.013 & C. 31 \\
\hline GRB111008A-1 & 2011-10-09 & $8.8 / 8.8 / 8.4$ & $1.0 / 0.9 / 0.9$ & $1.1-1.0$ & 1.3 & 8.5 & 21 & 4.990 & C. 32 \\
\hline GRB111008A-2 & 2011-10-10 & 8.0/8.0/7.2 & $1.0 / 0.9 / 0.9$ & $1.3-1.0$ & 0.9 & 20.1 & 22 & 4.990 & C. 32 \\
\hline GRB111107A & 2011-11-07 & $4.8 / 4.8 / 4.8$ & $1.0 / 0.9 / 0.9$ & $1.8-1.5$ & 0.8 & 5.3 & 21.5 & 2.893 & C. 33 \\
\hline GRB111117A ${ }^{f}$ & 2011-11-19 & $4.8 / 4.8 / 4.8$ & $1.0 / 0.9 / 0.9$ & $1.5-1.4$ & 0.7 & 38 & $>24$ & 2.211 & C. 34 \\
\hline GRB111123A-1 & 2011-11-24 & $6.2 / 6.6 / 6.6$ & $1.0 / 0.9 / 0.9$ & $1.6-1.1$ & 0.8 & 12.2 & $>24$ & 3.152 & C. 35 \\
\hline GRB111123A-2 $2^{c}$ & 2013-03-07 & $2.4 / 2.4 / 2.4$ & $1.0 / 0.9 / 0.9$ & $1.0-1.0$ & 0.5 & 11266 & $>24$ & 3.152 & C. 35 \\
\hline GRB111129A & 2011-11-30 & $3.6 / 3.6 / 3.6$ & $1.0 / 0.9 / 0.9$ & $1.6-2.1$ & 1.9 & 8.7 & $>24$ & 1.080 & C.36 \\
\hline
\end{tabular}

Notes. We here list the burst names and details of the spectroscopic observations. The exposure times and slit widths are given in the order $\mathrm{UVB} / \mathrm{VIS} / \mathrm{NIR}$. The column $\Delta t$ shows the time after trigger when the spectroscopic observation was started. Magacq gives the approximate magnitude (typically in the $R$-band) of the afterglow or the host in the acquisition image. ${ }^{(a)}$ Not part of the statistical sample. ${ }^{(b)}$ Spectrum dominated by light from the host galaxy. ${ }^{\left({ }^{(}\right)}$Spectrum of the host galaxy taken long after the burst. ${ }^{(d)}$ RRM observation. ${ }^{(e)}$ ADC malfunction during observation. ${ }^{(f)}$ Short burst. 
Table A.1. continued.

\begin{tabular}{|c|c|c|c|c|c|c|c|c|c|}
\hline GRB & Obs date & $\begin{array}{c}\text { Exptime } \\
(\mathrm{ks})\end{array}$ & $\begin{array}{c}\text { Slit width } \\
(\operatorname{arcsec})\end{array}$ & Airmass & $\begin{array}{l}\text { Seeing } \\
(\operatorname{arcsec})\end{array}$ & $\begin{array}{l}\Delta t \\
(\mathrm{~h})\end{array}$ & $\operatorname{Mag}_{\text {acq }}$ & Redshift & Notes \\
\hline GRB111209A-1 & 2011-12-10 & $4.8 / 4.8 / 4.8$ & $1.0 / 0.9 / 0.9$ & $1.1-1.2$ & 0.8 & 17.7 & 20.1 & 0.677 & C. 37 \\
\hline GRB111209A-2 & 2011-12-29 & 9.6/9.6/9.6 & $1.0 / 0.9 / 0.9$ & $1.2-2.0$ & 1.0 & 497 & 23 & 0.677 & C. 37 \\
\hline GRB111211A ${ }^{a}$ & $2011-12-13$ & $2.4 / 2.4 / 2.4$ & $1.0 / 0.9 / 0.9$ & $1.4-1.6$ & 0.6 & 31 & 19.5 & 0.478 & C. 38 \\
\hline GRB111228A & $2011-12-29$ & $2.4 / 2.4 / 2.4$ & $1.0 / 0.9 / 0.9$ & $1.4-1.4$ & 0.7 & 15.9 & 20.1 & 0.716 & C. 39 \\
\hline $\mathrm{GRB}_{120118 \mathrm{~B}^{c}}$ & $2013-02-13$ & $3.6 / 3.6 / 3.6$ & $1.0 / 0.9 / 0.9$ & $1.1-1.0$ & 0.7 & 9393 & $>24$ & 2.943 & C. 40 \\
\hline GRB120119A-1 & 2012-01-19 & $2.4 / 2.4 / 2.4$ & $1.0 / 0.9 / 0.9$ & $1.1-1.1$ & 0.6 & 1.4 & 17 & 1.728 & C. 41 \\
\hline GRB120119A-2 & 2012-01-19 & $1.2 / 1.2 / 1.2$ & $1.0 / 0.9 / 0.9$ & $1.8-1.9$ & 0.5 & 4.5 & 20 & 1.728 & C.41 \\
\hline GRB120119A-3 ${ }^{c}$ & $2013-02-26$ & $4.8 / 4.8 / 4.8$ & $1.0 / 0.9 / 0.6 \mathrm{JH}$ & $1.0-1.1$ & 1.8 & 9694 & $>24$ & 1.728 & C. 41 \\
\hline GRB120211A-1 ${ }^{c}$ & 2013-02-17 & $4.8 / 4.8 / 4.8$ & $1.0 / 0.9 / 0.9$ & $1.1-1.4$ & 1.3 & 8919 & $>24$ & 2.346 & C. 42 \\
\hline GRB $120211 \mathrm{~A}-2^{c}$ & $2013-03-20$ & $3.6 / 3.6 / 3.6$ & $1.0 / 0.9 / 0.9$ & $1.1-1.2$ & 1.2 & 9660 & $>24$ & 2.346 & C. 42 \\
\hline GRB120224A & $2012-02-25$ & $2.4 / 2.4 / 2.4$ & $1.0 / 0.9 / 0.9$ & $1.7-2.1$ & 1.3 & 19.8 & 22.3 & 1.10 & C. 43 \\
\hline GRB $120311 A^{a}$ & 2012-03-11 & $2.4 / 2.4 / 2.4$ & $1.0 / 0.9 / 0.9$ & $1.6-1.4$ & 0.7 & 3.7 & 21.6 & 0.350 & C. 44 \\
\hline GRB120327A-1 ${ }^{a}$ & $2012-03-27$ & $2.4 / 2.4 / 2.4$ & $1.0 / 0.9 / 0.9$ & $1.6-1.4$ & 0.6 & 2.1 & 18.8 & 2.815 & C. 45 \\
\hline GRB120327A-2 ${ }^{a}$ & $2012-03-28$ & $4.2 / 4.2 / 4.2$ & $1.0 / 0.9 / 0.9$ & $1.0-1.1$ & 0.6 & 29 & 22.5 & 2.815 & C. 45 \\
\hline GRB 120404A & $2012-04-05$ & $9.6 / 9.6 / 9.6$ & $1.0 / 0.9 / 0.9 \mathrm{JH}$ & $1.7-1.3$ & 1.3 & 15.7 & 21.3 & 2.876 & C. 46 \\
\hline GRB120422A & 2012-04-22 & $4.8 / 4.8 / 4.8$ & $1.0 / 0.9 / 0.9$ & $1.3-1.3$ & 0.7 & 16.5 & 22 & 0.283 & C. 47 \\
\hline GRB120712A & $2012-07-13$ & $4.8 / 4.8 / 4.8$ & $1.0 / 0.9 / 0.9$ & $1.5-2.5$ & 1.5 & 10.4 & 21.5 & 4.175 & C. 48 \\
\hline GRB120714B & $2012-07-15$ & $4.8 / 4.8 / 4.8$ & $1.0 / 0.9 / 0.9 \mathrm{JH}$ & $1.5-1.2$ & 1.2 & 7.8 & 22.1 & 0.398 & C. 49 \\
\hline $\mathrm{GRB}^{2} 20716 \mathrm{~A}^{a}$ & 2012-07-19 & $3.6 / 3.6 / 3.6$ & $1.0 / 0.9 / 0.9 \mathrm{JH}$ & $1.8-2.6$ & 1.1 & 62 & 20.9 & 2.486 & C. 50 \\
\hline $\mathrm{GRB}_{120722 \mathrm{~A}^{b}}$ & $2012-07-22$ & $4.8 / 4.8 / 4.8$ & $1.0 / 0.9 / 0.9$ & $1.3-1.3$ & 1.2 & 10.3 & 23.6 & 0.959 & C.51 \\
\hline $\mathrm{GRB}_{120805 \mathrm{~A}^{b}}$ & 2012-08-14 & $3.6 / 3.6 / 3.6$ & $1.0 / 0.9 / 0.9 \mathrm{JH}$ & $1.3-1.7$ & 0.9 & 218 & $>24$ & 3.9 & C.52 \\
\hline $\mathrm{GRB}^{2} 20815 \mathrm{~A}^{a}$ & $2012-08-15$ & $2.4 / 2.4 / 2.4$ & $1.0 / 0.9 / 0.9$ & $1.3-1.4$ & 0.7 & 1.69 & 18.9 & 2.358 & C. .53 \\
\hline $\mathrm{GRB} 120909 \mathrm{~A}^{d}$ & 2012-09-09 & $1.2 / 1.2 / 1.2$ & $1.0 / 0.9 / 0.9$ & $1.6-1.6$ & 1.6 & 1.7 & 21 & 3.929 & C. 54 \\
\hline GRB120923A & $2012-09-23$ & $9.6 / 9.6 / 9.6$ & $1.0 / 0.9 / 0.9 \mathrm{JH}$ & $1.2-1.4$ & 1.0 & 18.5 & $>24$ & 7.84 & C. .55 \\
\hline GRB121024A & $2012-10-24$ & $2.4 / 2.4 / 2.4$ & $1.0 / 0.9 / 0.9$ & $1.2-1.1$ & 0.6 & 1.8 & 20 & 2.300 & C.56 \\
\hline GRB $121027 \mathrm{~A}$ & $2012-10-30$ & $8.4 / 8.4 / 8.4$ & $1.0 / 0.9 / 0.9$ & $1.3-1.3$ & 1.3 & 69.4 & 21.15 & 1.773 & C.57 \\
\hline GRB121201A & 2012-12-02 & $4.8 / 4.8 / 4.8$ & $1.0 / 0.9 / 0.9 \mathrm{JH}$ & $1.1-1.1$ & 1.1 & 12.9 & 23 & 3.385 & C. 58 \\
\hline GRB121229A & $2012-12-29$ & $4.8 / 4.8 / 4.8$ & $1.0 / 0.9 / 0.9 \mathrm{JH}$ & $1.4-1.2$ & 1.5 & 2 & 21.5 & 2.707 & C.59 \\
\hline $\mathrm{GRB} 130131 \mathrm{~B}^{c}$ & 2013-03-09 & 7.2/7.2/7.2 & $1.0 / 0.9 / 0.9 \mathrm{JH}$ & $1.3-1.6$ & 1.1 & 874 & $>24$ & 2.539 & C. 60 \\
\hline $\mathrm{GRB}_{130408 \mathrm{~A}^{a}}$ & 2013-04-08 & $1.2 / 1.2 / 1.2$ & $1.0 / 0.9 / 0.9$ & $1.0-1.0$ & 0.9 & 1.9 & 20 & 3.758 & C.61 \\
\hline GRB130418A & 2013-04-18 & $1.2 / 1.2 / 1.2$ & 1.0/0.9/0.9 & $1.4-1.3$ & 1.2 & 4.6 & 18.5 & 1.222 & C. 62 \\
\hline GRB $130427 \mathrm{~A}$ & 2013-04-28 & $1.2 / 1.2 / 1.2$ & $1.0 / 0.9 / 0.9 \mathrm{JH}$ & $1.8-1.8$ & 0.8 & 16.5 & 19 & 0.340 & C. 63 \\
\hline GRB130427B & 2013-04-28 & $1.2 / 1.2 / 1.2$ & $1.0 / 0.9 / 0.9 \mathrm{JH}$ & $1.2-1.0$ & 1.0 & 20.3 & 22.7 & 2.780 & C. 64 \\
\hline $\mathrm{GRB}_{130603 \mathrm{~B}}{ }^{f}$ & 2013-06-04 & $2.4 / 2.4 / 2.4$ & $1.0 / 0.9 / 0.9$ & $1.4-1.4$ & 1.1 & 8.2 & 21.5 & 0.356 & C. 65 \\
\hline GRB130606A & 2013-06-07 & 4.2/4.2/4.2 & $1.0 / 0.9 / 0.9 \mathrm{JH}$ & $1.7-1.9$ & 0.9 & 7.1 & 19 & 5.91 & C.66 \\
\hline GRB130612A & 2013-06-12 & $1.2 / 1.2 / 1.2$ & $1.0 / 0.9 / 0.9$ & $1.3-1.3$ & 1.5 & 1.1 & 21.5 & 2.006 & C.67 \\
\hline GRB130615A & 2013-06-15 & $1.2 / 1.2 / 1.2$ & $1.0 / 0.9 / 0.9$ & $2.1-2.2$ & 1.0 & 0.8 & 21 & 2.9 & C. 68 \\
\hline GRB $130701 \mathrm{~A}$ & 2013-07-01 & $1.2 / 1.2 / 1.2$ & $1.0 / 0.9 / 0.9 \mathrm{JH}$ & & 1.4 & 5.5 & 19.9 & & C.69 \\
\hline GRB130925A & $2013-09-25$ & $5.88 / 6.0 / 6.9$ & $1.0 / 0.9 / 0.9 \mathrm{JH}$ & $1.0-1.0$ & 0.6 & 3.5 & $>24$ & 0.347 & C.70 \\
\hline $\mathrm{GRB} 131011 \mathrm{~A}^{a}$ & 2013-10-13 & $4.5 / 4.5 / 4.5$ & $1.0 / 0.9 / 0.9$ & $1.1-1.1$ & 0.8 & 34.2 & $>24$ & 1.874 & C.71 \\
\hline GRB131030A & $2013-10-31$ & $3.6 / 3.6 / 3.6$ & $1.0 / 0.9 / 0.9$ & $1.1-1.1$ & 1.1 & 3.4 & 18.0 & 1.296 & C. 72 \\
\hline GRB131103A & 2013-11-05 & $2.4 / 2.4 / 2.4$ & $1.0 / 0.9 / 0.9 \mathrm{JH}$ & $1.1-1.1$ & 1.0 & 5.8 & 20.48 & 0.599 & C.73 \\
\hline GRB131105A & $2013-11-05$ & $4.8 / 4.8 / 4.8$ & $1.0 / 0.9 / 0.9$ & $1.3-1.4$ & 0.8 & 1.3 & 22.4 & 1.686 & C. 74 \\
\hline GRB131117A & 2013-11-17 & $4.8 / 4.8 / 4.8$ & $1.0 / 0.9 / 0.9 \mathrm{JH}$ & $1.3-1.2$ & 1.7 & 1.1 & 20 & 4.042 & C. 75 \\
\hline $\mathrm{GRB}_{131231 \mathrm{~A}^{a}}$ & 2014-01-01 & $2.4 / 2.4 / 2.4$ & $1.0 / 0.9 / 0.9 \mathrm{JH}$ & $1.4-1.3$ & 0.9 & 20.2 & 18.5 & 0.642 & C.76 \\
\hline $\mathrm{GRB} 140114 \mathrm{~A}^{c}$ & 2014-03-28 & $5.4 / 5.4 / 5.4$ & $1.0 / 0.9 / 0.9 \mathrm{JH}$ & $1.7-1.7$ & 1.2 & 1746 & $>24$ & 3.0 & C.77 \\
\hline $\mathrm{GRB}_{140213 \mathrm{~A}^{a}}$ & 2014-02-14 & $1.2 / 1.2 / 1.2$ & $1.0 / 0.9 / 0.9 \mathrm{JH}$ & & 0.7 & 5.8 & 19.5 & 1.208 & C.78 \\
\hline GRB140301A & 2014-03-02 & 7.2/7.2/7.2 & $1.0 / 0.9 / 0.9 \mathrm{JH}$ & $1.1-1.1$ & 0.9 & 9 & 23.1 & 1.416 & C.79 \\
\hline $\mathrm{GRB}_{140311 \mathrm{~A}^{a}}$ & 2014-03-13 & $7.6 / 6.3 / 8.4$ & $1.0 / 0.9 / 0.9 \mathrm{JH}$ & $1.2-1.2$ & 0.6 & 32.5 & $>24$ & 4.954 & C. 80 \\
\hline $\mathrm{GRB}_{140430 \mathrm{~A}^{a}}$ & 2014-04-30 & $1.2 / 1.2 / 1.2$ & $1.0 / 0.9 / 0.9$ & $2.0-1.8$ & 1.6 & 2.5 & 19 & 1.601 & C.81 \\
\hline GRB140506A-1 & 2014-05-07 & $4.8 / 4.8 / 4.8$ & $1.0 / 0.9 / 0.9$ & $1.3-1.4$ & 0.7 & 8.8 & 20.9 & 0.889 & C.82 \\
\hline GRB140506A-2 & $2014-05-08$ & $4.8 / 4.8 / 4.8$ & $1.0 / 0.9 / 0.9$ & $1.2-1.3$ & 0.7 & 32.9 & $>24$ & 0.889 & C.82 \\
\hline GRB140515A & 2014-05-16 & $4.8 / 4.8 / 4.8$ & $1.0 / 0.9 / 0.9$ & $1.3-1.3$ & 1.4 & 15.5 & $>24$ & 6.327 & C.83 \\
\hline GRB140614A & 2014-06-14 & $2.4 / 2.4 / 2.4$ & $1.0 / 0.9 / 0.9$ & $1.8-1.8$ & 0.7 & 3.8 & 21.5 & 4.233 & C.84 \\
\hline
\end{tabular}


Table A.1. continued.

\begin{tabular}{|c|c|c|c|c|c|c|c|c|c|}
\hline GRB & Obs date & $\begin{array}{c}\text { Exptime } \\
(\mathrm{ks})\end{array}$ & $\begin{array}{c}\text { Slit width } \\
(\operatorname{arcsec})\end{array}$ & Airmass & $\begin{array}{c}\text { Seeing } \\
(\operatorname{arcsec})\end{array}$ & $\begin{array}{l}\Delta t \\
(\mathrm{~h})\end{array}$ & $\operatorname{Mag}_{\text {acq }}$ & Redshift & Notes \\
\hline GRB140622A ${ }^{f}$ & 2014-06-22 & $1.2 / 1.2 / 1.2$ & $1.0 / 0.9 / 0.9$ & $1.4-1.3$ & 1.0 & 0.8 & $>24$ & 0.959 & C.85 \\
\hline $\mathrm{GRB} 141028 \mathrm{~A}^{a}$ & 2014-10-29 & $2.4 / 2.4 / 2.4$ & $1.0 / 0.9 / 0.9$ & $1.5-1.4$ & 1.0 & 15.4 & 20 & 2.332 & C.86 \\
\hline $\mathrm{GRB}_{141031 \mathrm{~A}^{a, c}}$ & $2015-01-29$ & $2.4 / 2.4 / 2.4$ & $1.0 / 0.9 / 0.9$ & $1.2-1.3$ & 0.8 & 10912 & $>24$ & - & C.87 \\
\hline GRB141109A-1 & 2014-11-09 & $2.4 / 2.4 / 2.4$ & $1.0 / 0.9 / 0.9 \mathrm{JH}$ & $1.5-1.7$ & 0.8 & 1.9 & 19.2 & 2.993 & C. 88 \\
\hline GRB141109A-2 & 2014-11-10 & $4.3 / 4.3 / 4.5$ & $1.0 / 0.9 / 0.9 \mathrm{JH}$ & $1.7-2.0$ & 0.8 & 25.4 & $>24$ & 2.993 & C. 88 \\
\hline $\mathrm{GRB}_{150206 \mathrm{~A}^{a}}$ & 2015-02-07 & $2.4 / 2.4 / 2.4$ & $1.0 / 0.9 / 0.9$ & $2.1-1.9$ & 0.8 & 10 & 21.9 & 2.087 & C.89 \\
\hline GRB150301B & $2015-03-02$ & $3.6 / 3.6 / 3.6$ & $1.0 / 0.9 / 0.9 \mathrm{JH}$ & $1.2-1.2$ & 1.1 & 5.1 & 21.0 & 1.517 & C. 90 \\
\hline GRB150403A & 2015-04-04 & $2.4 / 2.4 / 2.4$ & 1.0/0.9/0.9 & $1.6-1.7$ & 0.7 & 10.8 & 19.1 & 2.057 & C.91 \\
\hline $\mathrm{GRB}_{150423 \mathrm{~A}^{d, f}}$ & $2015-04-23$ & $4.8 / 4.8 / 4.8$ & $1.0 / 0.9 / 0.9$ & $2.7-2.4$ & 1.4 & 0.4 & $>24$ & 1.394 & C.92 \\
\hline GRB150428A & $2015-04-28$ & $2.4 / 2.4 / 2.4$ & $1.0 / 0.9 / 0.9 \mathrm{JH}$ & $1.6-1.5$ & 0.8 & 3.7 & $>24$ & - & C.93 \\
\hline $\mathrm{GRB}_{150514 \mathrm{~A}^{a}}$ & $2015-05-15$ & $2.4 / 2.4 / 2.4$ & $1.0 / 0.9 / 0.9$ & $2.3-2.1$ & 0.9 & 28.4 & 19.5 & 0.807 & C.94 \\
\hline $\mathrm{GRB}_{150518 \mathrm{~A}^{a}}$ & $2015-05-20$ & $2.4 / 2.4 / 2.4$ & $1.0 / 0.9 / 0.9 \mathrm{JH}$ & $1.3-1.3$ & 1.7 & 30.7 & $>24$ & 0.256 & C.95 \\
\hline $\mathrm{GRB}_{150616 \mathrm{~A}^{a, c}}$ & $2015-09-12$ & $2.4 / 2.4 / 2.4$ & $1.0 / 0.9 / 0.9 \mathrm{JH}$ & $1.2-1.1$ & 1.2 & 2092 & $>24$ & 1.188 & C.96 \\
\hline GRB150727A & $2015-07-28$ & $3.6 / 3.6 / 3.6$ & $1.0 / 0.9 / 0.9 \mathrm{JH}$ & $1.2-1.2$ & 1.4 & 5.0 & 20.5 & 0.313 & C.97 \\
\hline GRB150821A ${ }^{d}$ & $2015-08-21$ & $2.4 / 2.4 / 2.4$ & $1.0 / 0.9 / 0.9$ & $2.0-1.8$ & 1.3 & 0.2 & 16 & 0.755 & C.98 \\
\hline GRB150910A & 2015-09-11 & $1.8 / 1.8 / 1.8$ & $1.0 / 0.9 / 0.9 \mathrm{JH}$ & $1.9-1.9$ & 1.3 & 20.1 & 21.2 & 1.359 & C.99 \\
\hline GRB150915A & 2015-09-16 & $4.8 / 4.8 / 4.8$ & $1.0 / 0.9 / 0.9 \mathrm{JH}$ & $1.1-1.1$ & 1.6 & 3.3 & 23 & 1.968 & C. 100 \\
\hline GRB151021 $\mathrm{A}^{d}$ & $2015-10-21$ & 4.2/4.2/4.2 & $1.0 / 0.9 / 0.9$ & $1.0-1.1$ & 1.4 & 0.75 & 18.2 & 2.330 & C. 101 \\
\hline GRB151027B & $2015-10-28$ & $2.4 / 2.4 / 2.4$ & $1.0 / 0.9 / 0.9 \mathrm{JH}$ & $1.5-1.7$ & 1.2 & 5 & 20.5 & 4.063 & C. 102 \\
\hline GRB151029A & $2015-10-29$ & $1.2 / 1.2 / 1.2$ & $1.0 / 0.9 / 0.9 \mathrm{JH}$ & $1.9-1.7$ & 1.1 & 1 & 20 & 1.423 & C. 103 \\
\hline $\mathrm{GRB}_{151031 \mathrm{~A}^{d}}$ & $2015-10-31$ & $4.2 / 4.2 / 4.2$ & $1.0 / 0.9 / 0.9$ & $1.1-1.1$ & 1.1 & 0.3 & 20.4 & 1.167 & C.104 \\
\hline GRB160117B & $2016-01-18$ & $4.8 / 4.8 / 4.8$ & $1.0 / 0.9 / 0.9 \mathrm{JH}$ & $1.1-1.2$ & 1.1 & 13.5 & 20.8 & 0.870 & C. 105 \\
\hline $\mathrm{GRB} 160203 \mathrm{~A}^{d}$ & 2016-02-03 & $6.6 / 6.6 / 6.6$ & $1.0 / 0.9 / 0.9$ & $1.0-1.8$ & 1.0 & 0.3 & 18 & 3.518 & C.106 \\
\hline $\mathrm{GRB} 160228 \mathrm{~A}^{c}$ & 2016-03-12 & $4.8 / 4.8 / 4.8$ & $1.0 / 0.9 / 0.9 \mathrm{JH}$ & $1.7-1.7$ & 1.0 & 296 & $>24$ & 1.640 & C.107 \\
\hline GRB160303A ${ }^{f}$ & 2016-03-04 & $4.8 / 4.8 / 4.8$ & $1.0 / 0.9 / 0.9 \mathrm{JH}$ & $1.6-1.5$ & 0.8 & 19.1 & $>24$ & - & C. 108 \\
\hline GRB160314A & $2016-03-15$ & $4.8 / 4.8 / 4.8$ & $1.0 / 0.9 / 0.9 \mathrm{JH}$ & $1.3-1.3$ & 0.8 & 13.0 & 21.7 & 0.726 & C.109 \\
\hline $\mathrm{GRB}_{160410 \mathrm{~A}^{d, f}}$ & 2016-04-10 & $1.8 / 1.8 / 1.8$ & $1.0 / 0.9 / 0.9$ & $2.5-2.3$ & 0.5 & 0.15 & 20.3 & 1.717 & C. 110 \\
\hline GRB160425A & $2016-04-26$ & $4.8 / 4.8 / 4.8$ & $1.0 / 0.9 / 0.9 \mathrm{JH}$ & $1.3-1.3$ & 0.5 & 7.2 & 21.1 & 0.555 & C. 111 \\
\hline $\mathrm{GRB}^{160625 B^{a}}$ & $2016-06-27$ & $2.4 / 2.4 / 2.4$ & $1.0 / 0.9 / 0.9 \mathrm{JH}$ & $1.3-1.3$ & 0.7 & 30 & 19.1 & 1.406 & C. 112 \\
\hline GRB160804A-1 $^{a}$ & 2016-08-04 & $2.4 / 2.4 / 2.4$ & $1.0 / 0.9 / 0.9 \mathrm{JH}$ & $1.4-1.3$ & 0.6 & 22.4 & 21.2 & 0.736 & C. 113 \\
\hline GRB160804A-2 ${ }^{a, c}$ & 2016-08-27 & $3.6 / 3.6 / 3.6$ & $1.0 / 0.9 / 0.9 \mathrm{JH}$ & $1.9-1.8$ & 0.6 & 574 & $>24$ & 0.736 & C. 113 \\
\hline GRB161001A & 2016-10-01 & $2.4 / 2.4 / 2.4$ & $1.0 / 0.9 / 0.9 \mathrm{JH}$ & $1.2-1.3$ & 0.5 & 6.1 & $>24$ & 0.891 & C. 114 \\
\hline $\mathrm{GRB} 61007 \mathrm{~A}^{c}$ & $2016-10-14$ & $2.4 / 2.4 / 2.4$ & $1.0 / 0.9 / 0.9 \mathrm{JH}$ & $1.6-1.6$ & 0.7 & 323 & $>24$ & - & C. 115 \\
\hline GRB161014A & $2016-10-15$ & $4.8 / 4.8 / 4.8$ & $1.0 / 0.9 / 0.9 \mathrm{JH}$ & $1.1-1.2$ & 0.5 & 11.6 & 21.4 & 2.823 & C. 116 \\
\hline $\mathrm{GRB}_{161023 \mathrm{~A}^{a}}$ & $2016-10-24$ & $1.2 / 1.2 / 1.2$ & $1.0 / 0.9 / 0.9 \mathrm{JH}$ & $1.2-1.2$ & 0.9 & 3 & 17.5 & 2.710 & C. 117 \\
\hline GRB161117A & 2016-11-17 & $2.4 / 2.4 / 2.4$ & 1.0/0.9/0.9 & $1.8-1.6$ & 2.6 & 0.73 & 19 & 1.549 & C. 118 \\
\hline GRB161219B & 2016-12-21 & $2.4 / 2.4 / 2.4$ & $1.0 / 0.9 / 0.9 \mathrm{JH}$ & $1.1-1.1$ & 0.9 & 35.7 & 19.5 & 0.146 & C. 119 \\
\hline GRB170113A & 2017-01-14 & $4.8 / 4.8 / 4.8$ & $1.0 / 0.9 / 0.9 \mathrm{JH}$ & $1.5-1.4$ & 0.9 & 15.23 & 21.7 & 1.968 & C. 120 \\
\hline GRB170202A & 2017-02-03 & $2.4 / 2.4 / 2.4$ & $1.0 / 0.9 / 0.9 \mathrm{JH}$ & $1.3-1.2$ & 0.7 & 9.7 & 20.8 & 3.645 & C. 121 \\
\hline
\end{tabular}



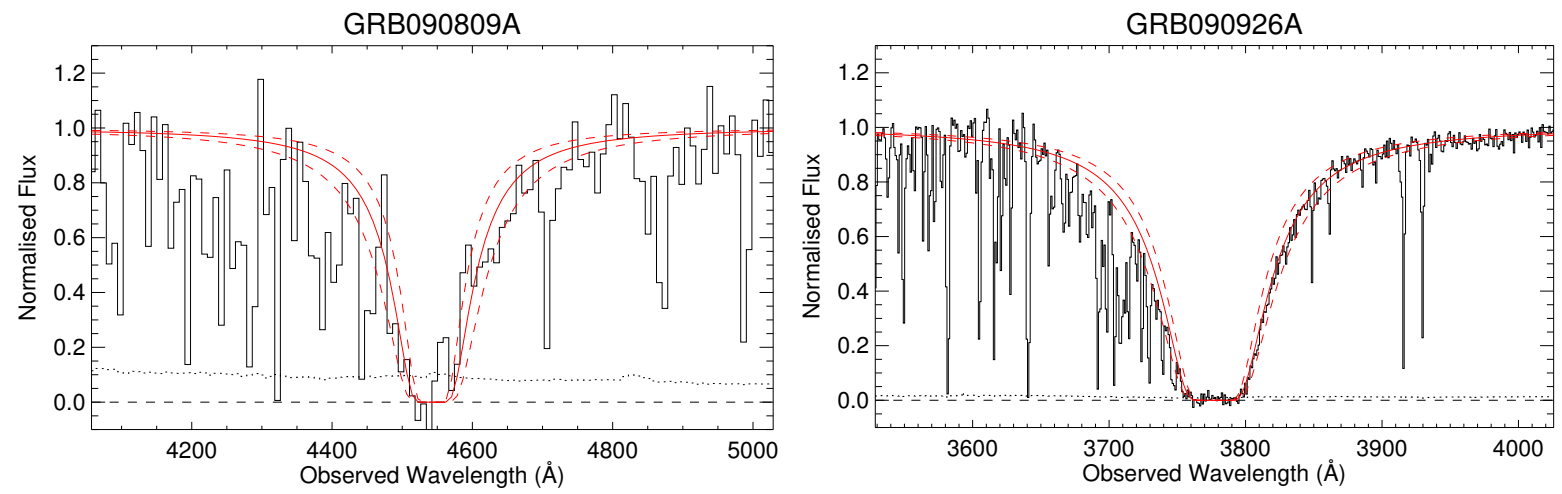

GRB 100219A
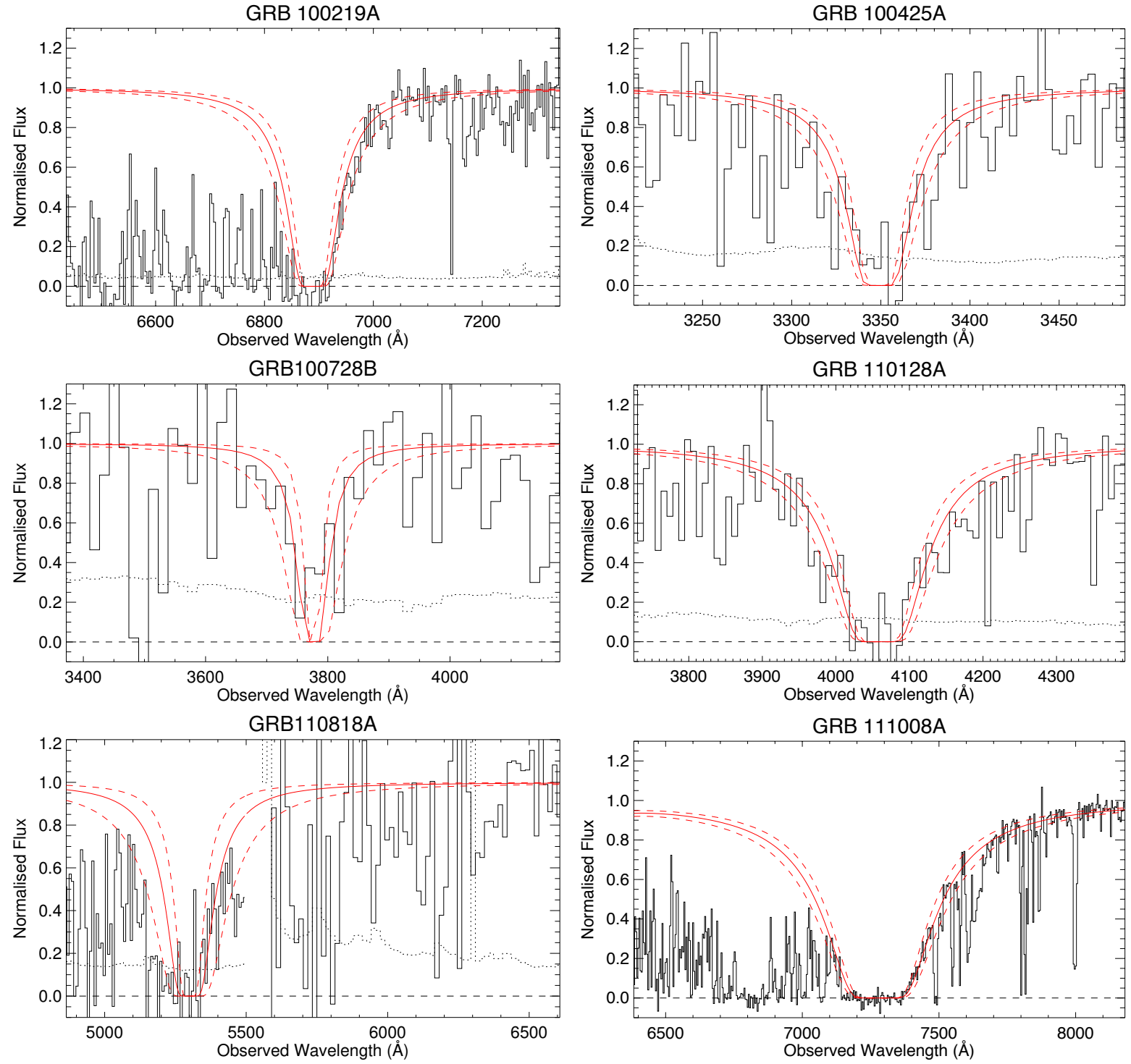

Fig. A.1. Measurements of the hydrogen column densities for all bursts with a clear Lyman alpha absorption system. In solid black is shown the spectrum with black dotted giving the corresponding 1- $\sigma$ error. The black dashed line shows zero flux density. The solid red line is the absorption of column density equal to the value presented in Table 3 with the $1-\sigma$ interval shown with dashed lines. The presence of Ly $\alpha$, as noted in Table 3 , is indicated with a black arrow. 

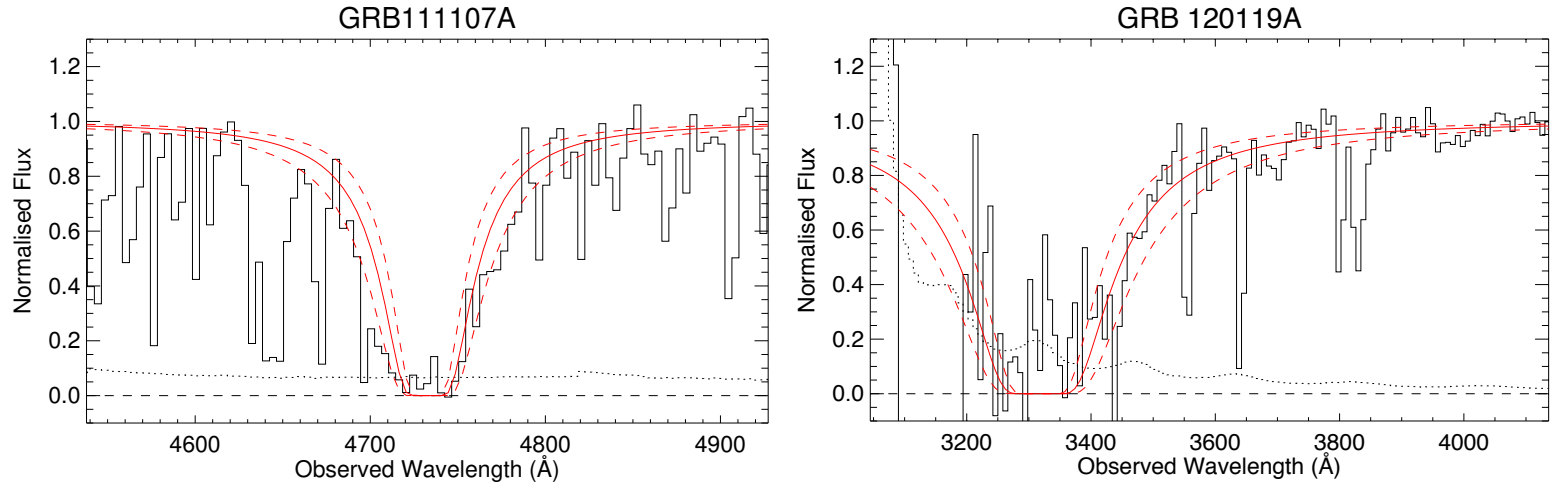

GRB 120327A
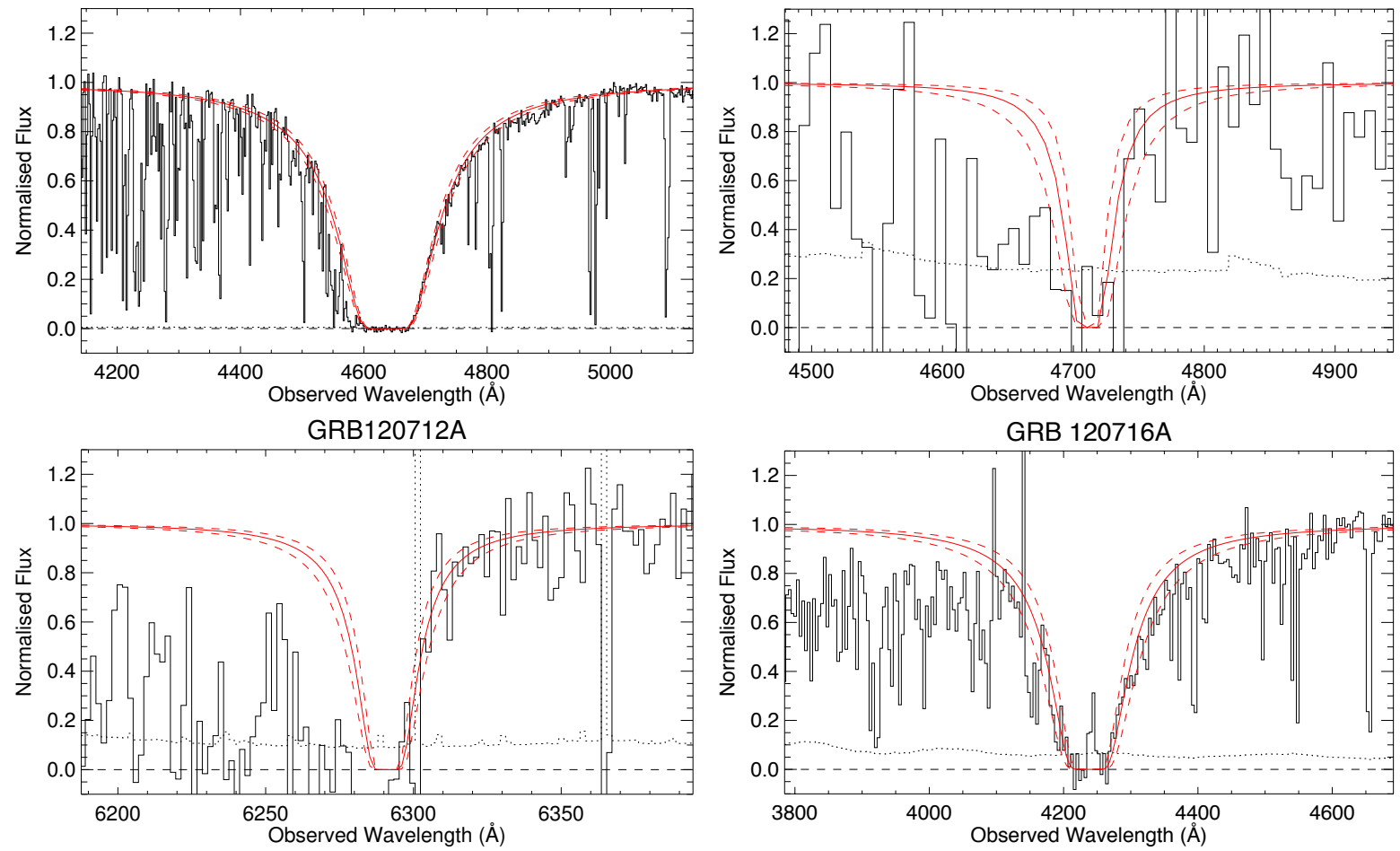

GRB 120815A

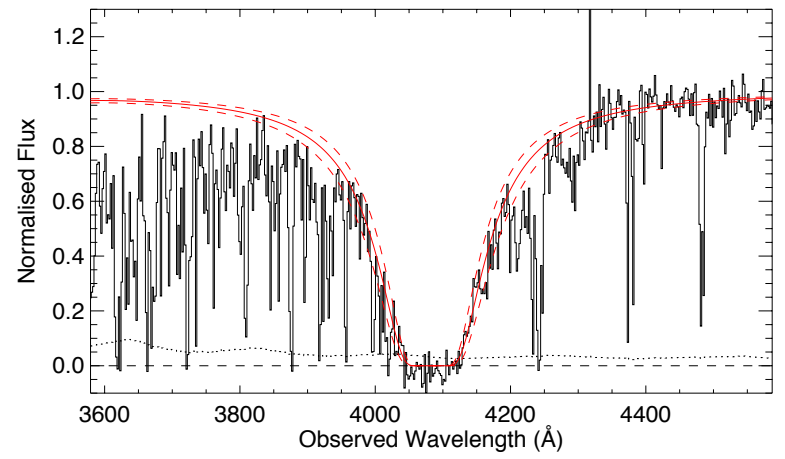

GRB 120909A

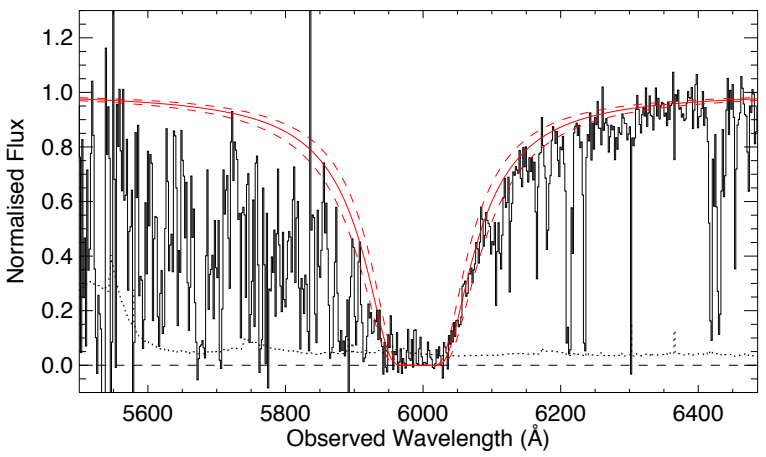

Fig. A.1. continued. 

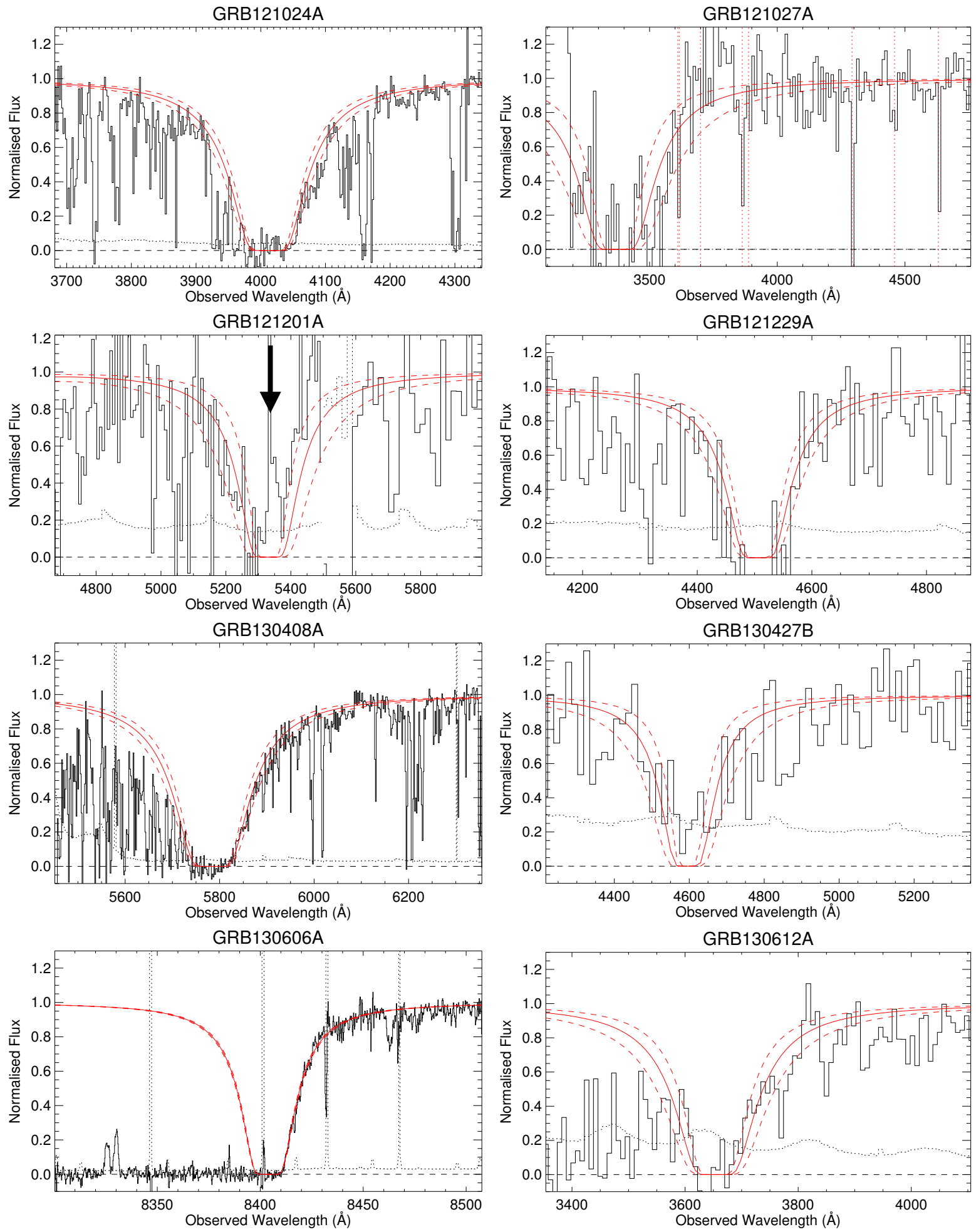

Fig. A.1. continued. 
J. Selsing et al.: The X-shooter GRB afterglow legacy sample
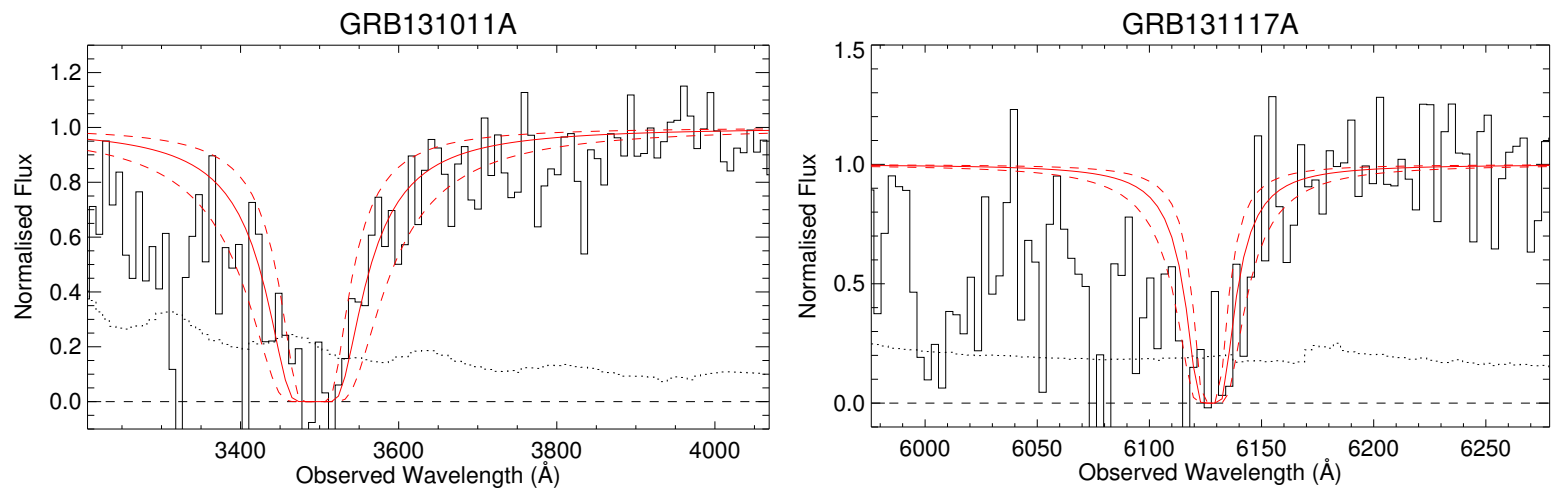

GRB 140311A
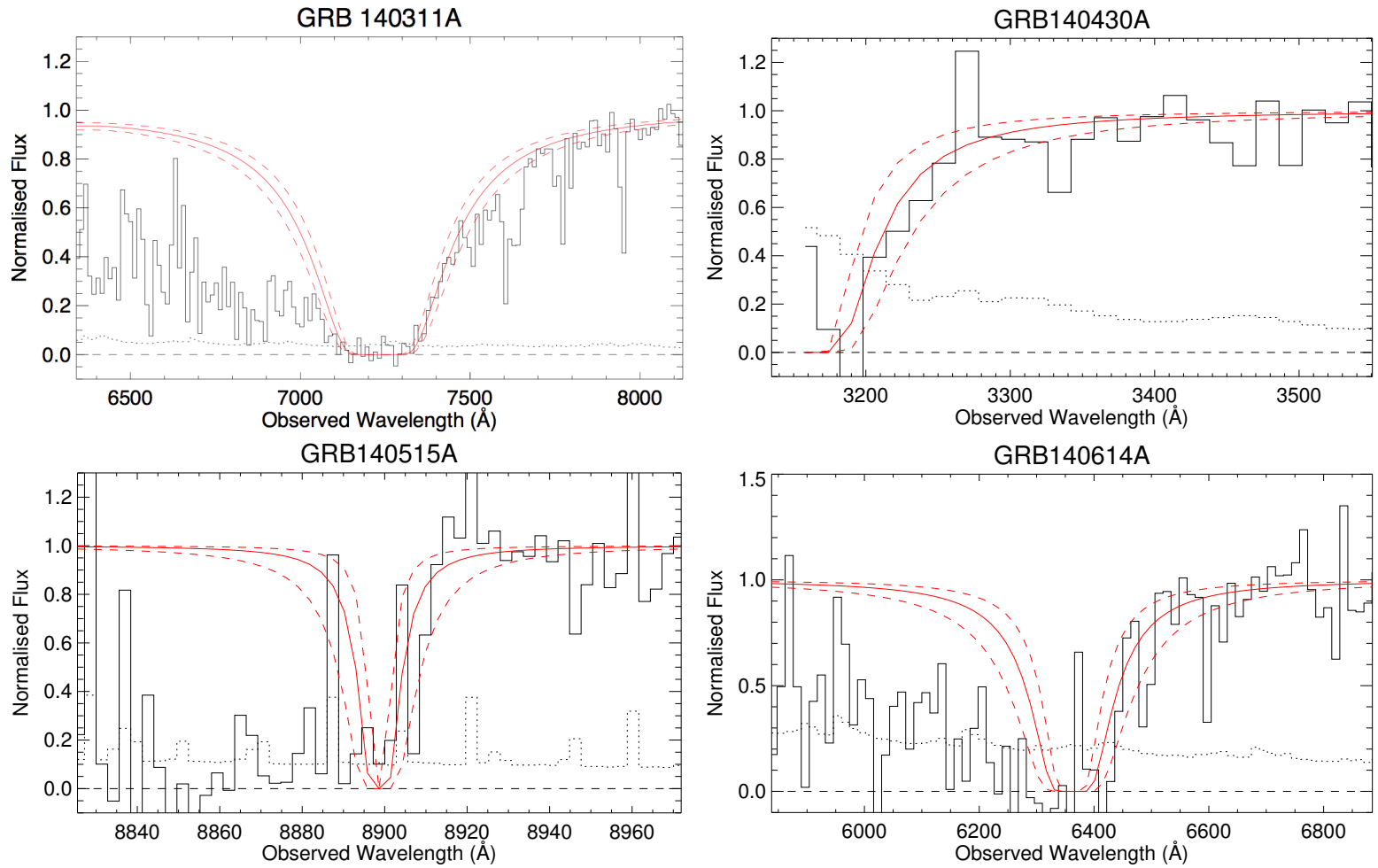

GRB141028A

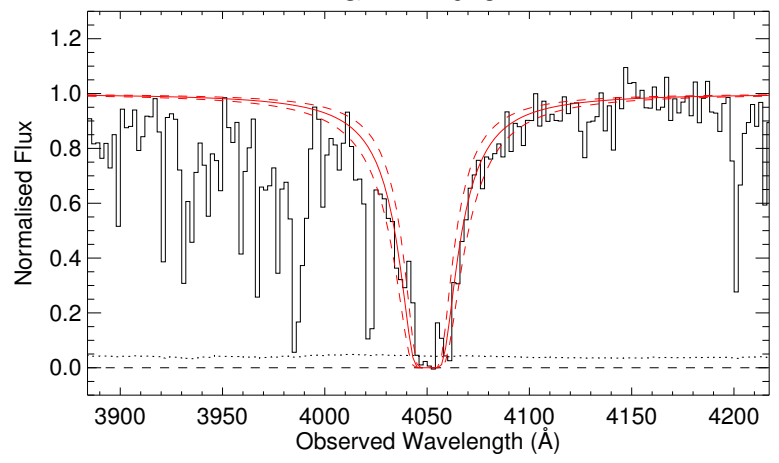

GRB141109A

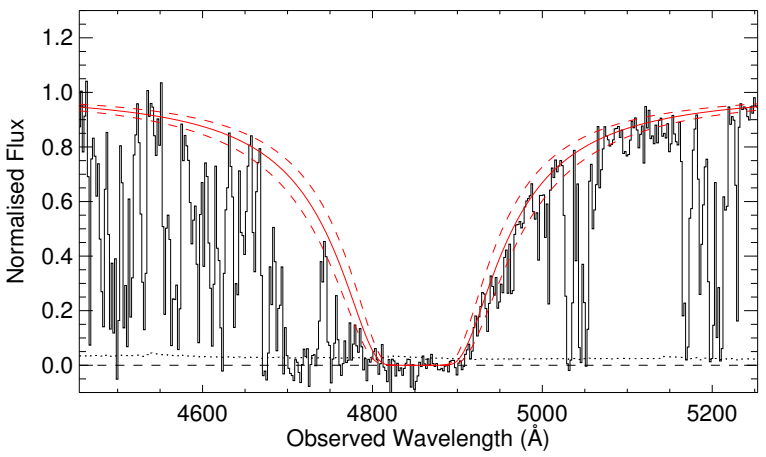

Fig. A.1. continued. 

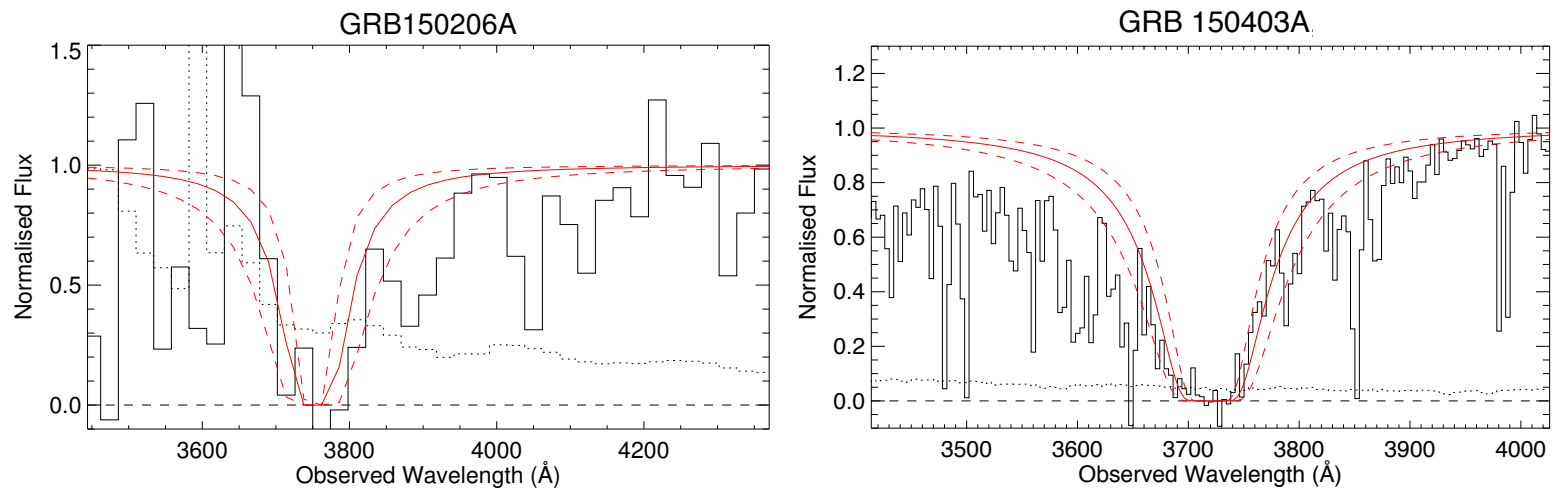

GRB150915A
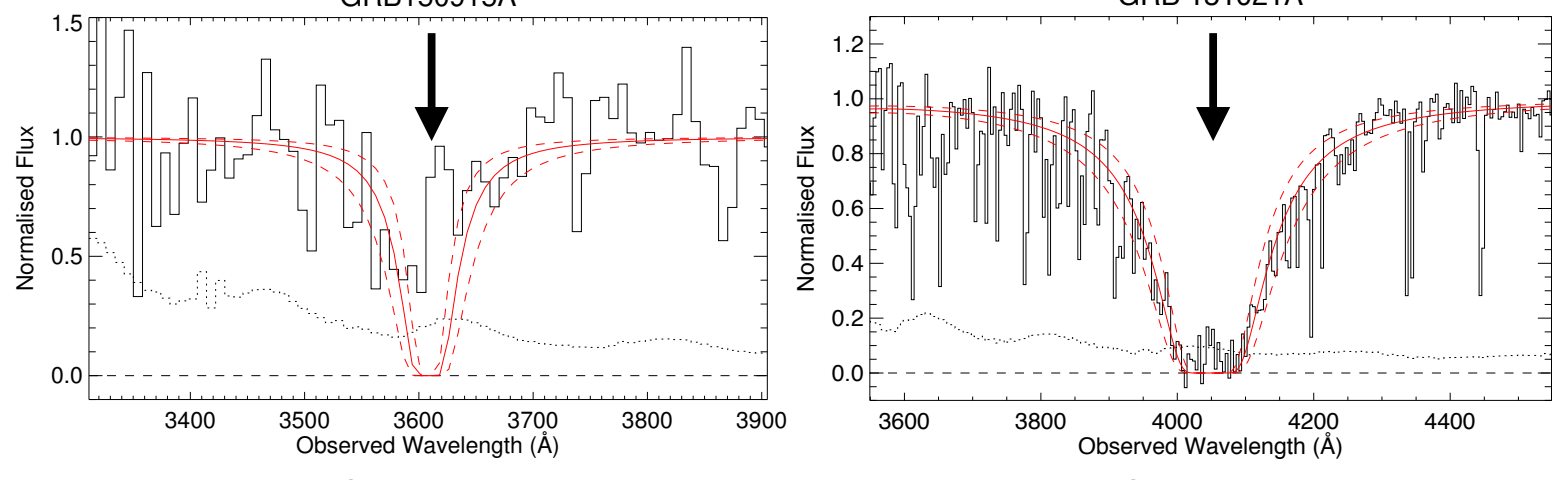

GRB151027B

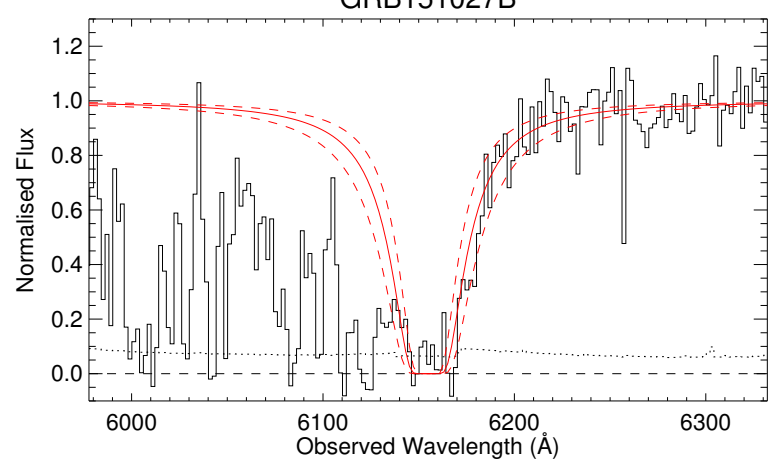

GRB160203A

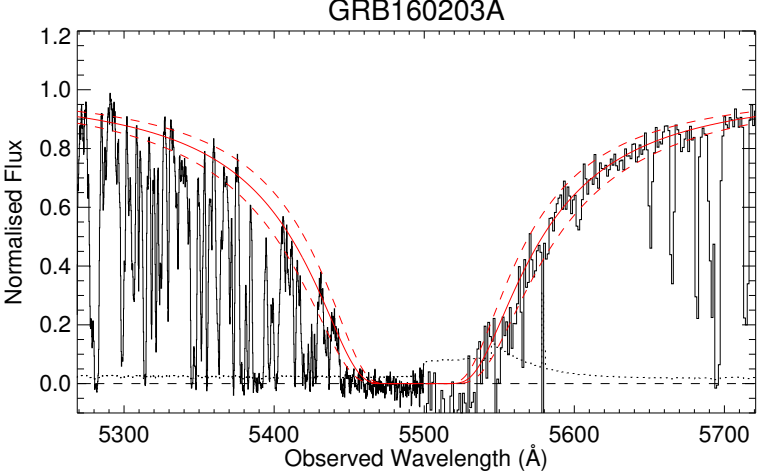

GRB 160410A

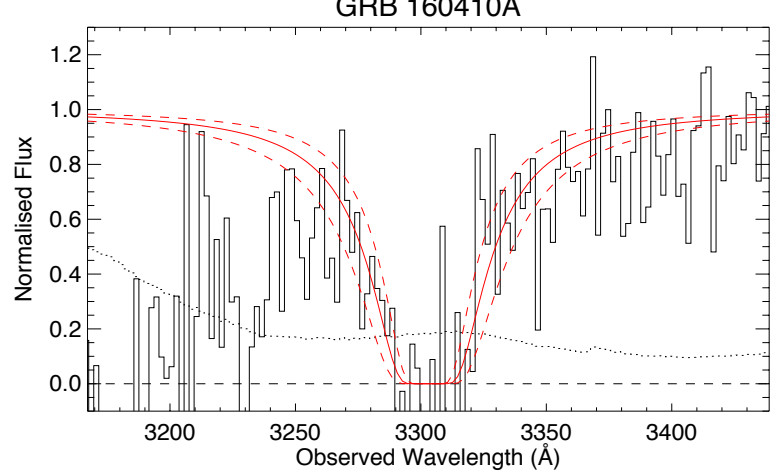

GRB161014A

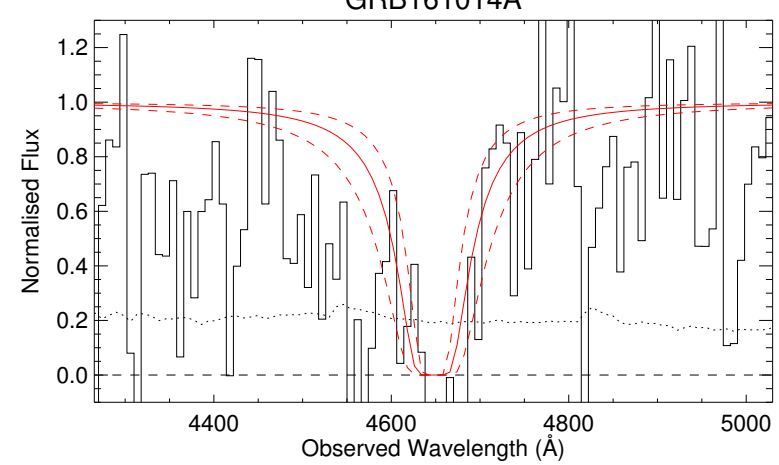

Fig. A.1. continued. 

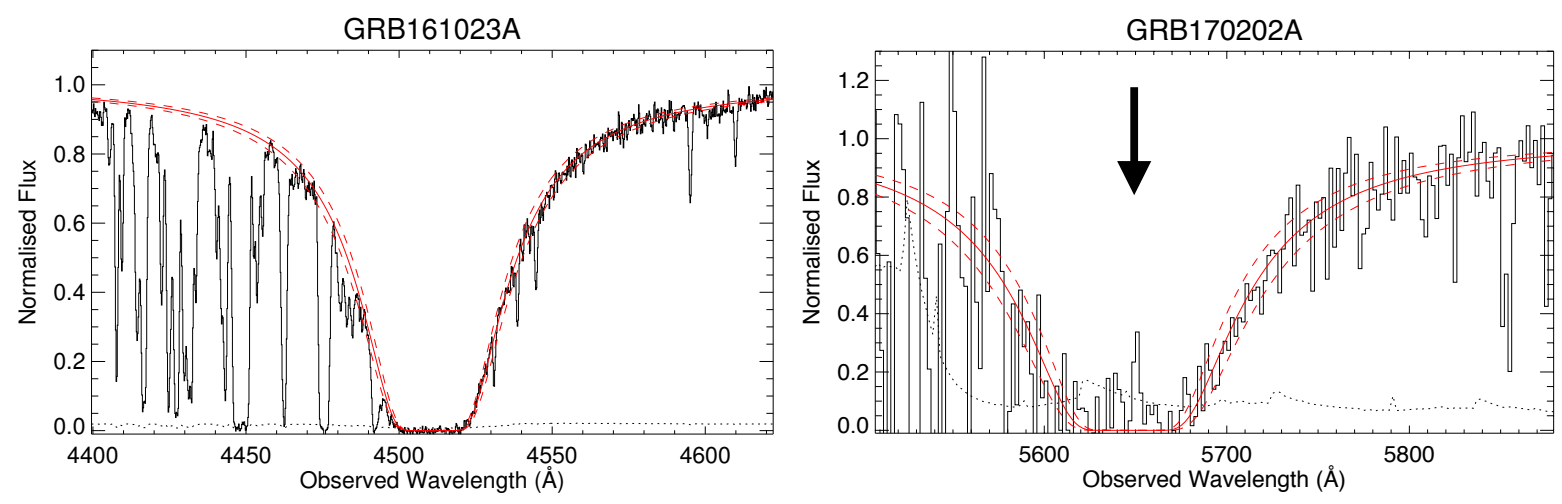

Fig. A.1. continued.

\section{Appendix B: The complex error function and the spectral PSF}

When modelling the spectral PSF, we need to evaluate the Voigtprofile. The Voigt profile, which is the convolution of the Gaussian and Lorentzian profiles, can, centred at zero, be written as (Pagnini \& Mainardi 2010)

$$
\begin{aligned}
V(\lambda, \sigma, \gamma) & =G(\lambda, \sigma) \otimes L(\lambda, \gamma) \\
& =\int_{-\infty}^{\infty} G(\xi, \sigma) L(\lambda-\xi, \gamma) \mathrm{d} \xi \\
& =\int_{-\infty}^{\infty} \frac{1}{\sqrt{2 \pi} \sigma} \mathrm{e}^{-\left(\frac{\xi}{\sqrt{2} \sigma}\right)^{2}} \frac{1}{\gamma \pi} \frac{\gamma^{2}}{(\lambda-\xi)^{2}+\gamma^{2}} \mathrm{~d} \xi \\
& =\frac{\gamma}{\sqrt{2} \sigma} \frac{1}{\pi^{3 / 2}} \int_{-\infty}^{\infty} \frac{\mathrm{e}^{-\left(\frac{\xi}{\sqrt{2} \sigma}\right)^{2}}}{(\lambda-\xi)^{2}+\gamma^{2}} \mathrm{~d} \xi .
\end{aligned}
$$

We can by making the following substitution, $\xi=\sqrt{2} \sigma$ and $\mathrm{d} \xi=\sqrt{2} \sigma \mathrm{d} t$, write it as

$$
\begin{aligned}
V(\lambda, \sigma, \gamma) & =\frac{\sqrt{2} \sigma}{\sqrt{\pi}} \frac{\frac{\gamma}{\sqrt{2} \sigma}}{\pi} \int_{-\infty}^{\infty} \frac{\mathrm{e}^{-t^{2}}}{(\lambda-\sqrt{2} \sigma t)^{2}+\gamma^{2}} \mathrm{~d} t \\
& =\frac{1}{\sqrt{2 \pi} \sigma} \frac{\frac{\gamma}{\sqrt{2} \sigma}}{\pi} \int_{-\infty}^{\infty} \frac{\mathrm{e}^{-t^{2}}}{\left(\frac{\lambda}{\sqrt{2} \sigma}-t\right)^{2}+\left(\frac{\gamma}{\sqrt{2} \sigma}\right)^{2}} \mathrm{~d} t .
\end{aligned}
$$

This form of the convolution is closely related to the complex probability function (Letchworth \& Benner 2007; Abrarov \& Quine 2015a),

$$
W(z)=\frac{i}{\pi} \int_{-\infty}^{\infty} \frac{\mathrm{e}^{-t^{2}}}{z-t} \mathrm{~d} t
$$

for any complex argument, $z=x+\mathrm{i} y$. The complex probability function can be expressed as a sum of a real and an imaginary part (Benner et al. 1995; Abrarov \& Quine 2015b),

$$
\begin{aligned}
W(x, y) & =K(x, y)+\mathrm{i} L(x, y) \\
& =\frac{y}{\pi} \int_{-\infty}^{\infty} \frac{\mathrm{e}^{-t^{2}}}{(x-t)^{2}+y^{2}} \mathrm{~d} t+\frac{\mathrm{i}}{\pi} \int_{-\infty}^{\infty} \frac{(x-t) \mathrm{e}^{-t^{2}}}{(x-t)^{2}+y^{2}} \mathrm{~d} t,
\end{aligned}
$$

where the real part, $\operatorname{Re}[W(x, y)]=\sqrt{2 \pi} \sigma V(\lambda, \sigma, \gamma)$ if $x=\frac{\lambda}{\sqrt{2} \sigma}$ and $y=\frac{\gamma}{\sqrt{2} \sigma}$, which can be obtained by using the complex argument, $z=\frac{\lambda+\mathrm{i} \gamma}{\sqrt{2} \sigma}$, in the complex probability function. If $\operatorname{Im}[z] \geq 0$, which is always guaranteed for the width of a spectral profile, the complex probability function equals the complex error function. The complex error function has numerous, fast, numerical approximations where in this work we use the scipy. special. wofz (Oliphant 2007) implementation.

\section{Appendix C: Notes on individual objects}

\section{C.1. $\operatorname{GRB} 090313(z=3.373)$}

The first GRB ever observed with X-shooter, during the commissioning of the instrument, these data formed the basis of GCN 9015 (de Ugarte Postigo et al. 2009a) and are published in de Ugarte Postigo et al. (2010). Due to the lingering brightness of GRB 090313, $6.9 \mathrm{ks}$ spectroscopic integration starting $45 \mathrm{~h}$ after the BAT trigger reveals a wealth of absorption features superposed on the afterglow continuum at a common redshift of $z=3.373$. Two intervening systems at $z=1.959$ and $z=1.800$ are identified based on strong $\mathrm{Mg}_{\mathrm{II}}$-absorption. Because this burst is observed before the instrument is science-verified, it does not enter into the statistical sample.

\section{C.2. GRB $090530(z=1.266)$}

Observed during the verification of the instrument, these data forms the basis of GCN 15571 (Goldoni et al. 2013), but are not published elsewhere. Observations began $20.6 \mathrm{~h}$ after the BAT trigger and $4.8 \mathrm{ks}$ spectroscopic integration in all three arms reveals the absorption signature for a host at $z=1.266$ from the detection of $\mathrm{Mg}_{\text {II, }} \mathrm{Mg}_{\text {I }}, \mathrm{Si}$ II, Fe II, Al III. Because this burst was observed before the instrument was finished completely, it does not enter into the statistical sample.

\section{C.3. $\operatorname{GRB} 090809(z=2.737)$}

Observed during the first instrument testing period and was reported in GCN 9761 (Malesani et al. 2009a) and is additionally used as the basis for the master thesis by Ása Skúladóttir (2010). $7.2 \mathrm{ks}$ integration starting $10.2 \mathrm{~h}$ after the GRB trigger yields a clear afterglow continuum in all arms. The simultaneous detection of absorption lines identified as Ly $\alpha, \mathrm{Si}$ II, O I, Si I*, $\mathrm{Si}$ IV, $\mathrm{C}_{\text {IV }}, \mathrm{Fe}$ II, $\mathrm{Al}$ II, $\mathrm{Al}$ III and $\mathrm{Mg}$ II at $z=2.737$ sets it as the redshift of the GRB. Because this burst is observed before the instrument was science-verified, it does not enter into the statistical sample. 


\section{C.4. GRB 090926A $(z=2.106)$}

Obtained during the second instrument testing period, this dataset forms the basis of GCN 9942 (Malesani et al. 2009b) and is additionally published in D'Elia et al. (2010). Spectroscopic integration started 22 hours after the BAT trigger and from the acquisition camera the optical afterglow is measured to $R=17.9 \mathrm{mag}$ at the beginning of the observations which causes a strong continuum to be seen in all arms. An absorption trough due to $\operatorname{Ly} \alpha$ is clearly visible along with numerous metal resonance lines $\mathrm{C}$ IV, Si II, Si II* Fe II, Mg II, all at $z=2.106$. Because this burst was observed before the instrument was science-verified, it does not enter into the statistical sample.

\section{C.5. GRB $091018(z=0.971)$}

The first burst observed during normal operation and the first burst that enters the statistical sample. These data are the basis for GCN 10042 (de Ugarte Postigo et al. 2009b) and is published in Wiersema et al. (2012a). With a bright afterglow and a rapid follow-up, this spectrum is of pristine quality. The afterglow continuum is bright throughout all spectroscopic arms which allows the ready detection of $\mathrm{Al}$ II, $\mathrm{Al}$ III, Fe II, Mn II, Mg II, Mg I, and Ca II - all located at $z=0.971$, setting it as the redshift of the GRB.

\section{C.6. GRB $091127(z=0.490)$}

Obtained 4 days after the burst trigger, these data forms the basis for GCN 10233 (Thöne et al. 2009) and are published in Vergani et al. (2011a). Due to the late follow-up and a nearby moon, the $\mathrm{S} / \mathrm{N}$ of the afterglow continuum is low, especially in the UVB arm. This is why no clear absorption lines are detected against the afterglow continuum, although see Vergani et al. (2011a) which report a tentative detection of $\mathrm{Mg}$ II. Emission lines from the underlying host are clearly visible with lines from [O II], $\mathrm{H} \beta$, [O III] , and $\mathrm{H} \alpha$ all at $z=0.490$. This bursts is additionally associated with SN2009nz (Cobb et al. 2010; Berger et al. 2011; Olivares et al. 2015).

\section{C.7. GRB 100205A $(z=n a)$}

Observed 3 days after the Swift trigger. No afterglow or host detected is in $10.8 \mathrm{ks}$. The GRB is likely located at high redshift (GCN GCN10399; Perley et al. 2010). The spectrum has not otherwise been published previously.

\section{C.8. GRB 100219A $(z=4.667)$}

These data presented here have also formed the basis of GCN 10441 (Groot et al. 2010) and are published in Thöne et al. (2013). Observations started $12.5 \mathrm{~h}$ after the Swift trigger and has a total exposure time of $4.8 \mathrm{ks}$. Absorption features, including those of Ly $\alpha$ and from a multitude of ions are detected against the afterglow continuum at $z=4.667$. Additionally, absorption from an intervening system is found at $z=2.181$.

\section{C.9. $G R B 100316 B(z=1.180)$}

The data presented here also formed the basis of GCN 10495 (Vergani et al. 2010a). The spectrum has not otherwise been published. Observations started $44 \mathrm{~min}$ after the Swift trigger and have a total exposure time of $2.4 \mathrm{ks}$. Absorption features from
Fe II, $\mathrm{Al}$ II, $\mathrm{Al}_{\text {IIII, }} \mathrm{Mg}_{\text {II }}$ and $\mathrm{Mg}_{\mathrm{I}}$ are well detected against the afterglow continuum at $z=1.180$. Additionally, strong absorption lines from Fe II and $\mathrm{Mg}$ II from an intervening system are found at $z=1.063$.

\section{C.10. GRB 100316D $(z=0.059)$}

The data presented here also formed the basis of GCN 10512 (Vergani et al. 2010b), GCN 10513 (Vergani et al. 2010c), GCN 10543 (Bufano et al. 2010) and are published in Bufano et al. (2012) and Starling et al. (2011). This GRB is very close by and has an associated SN, SN2010bh, and has therefore undergone intense follow-up (Olivares et al. 2012; Cano et al. 2011; Izzo et al. 2017). The spectra presented here consists of a subset of the entire VLT/X-shooter campaign, covering the four first observing days while the afterglow still contributes significantly to the total emission. The first observations started $10 \mathrm{~h}$ after the burst, before the SN was discovered, and targeted the star-forming "A"-region (Starling et al. 2011), not the GRB. A very rich spectrum containing a multitude of emission lines puts the host at $z=0.059$. For three consecutive nights, 58, 79 and $101 \mathrm{~h}$ after the Swift trigger, the afterglow was observed as it transitioned into the spectrum of a high-velocity Ic-BL SN. The observations taken 79 and $101 \mathrm{~h}$ after the burst are taken under programme 084.D0265(A) (PI: Benetti), but with an identical setup to the first two observations.

\section{C.11. GRB 100418A $(z=0.624)$}

The data presented here also formed the basis of GCN 10620 (Antonelli et al. 2010) and GCN 10631 (Malesani \& Palazzi 2010) and are published in de Ugarte Postigo et al. (2011a). The burst has been followed up in three epochs of observations, $0.4,1.4$, and 2.4 days after the burst, each lasting $4.8 \mathrm{ks}$. The unambiguous redshift of the host, $z=0.624$, is found from the simultaneous detection of emission features belonging to nebular lines, including $\mathrm{HI}_{\mathrm{I}},\left[\mathrm{O}_{\mathrm{II}}\right],\left[\mathrm{O}_{\mathrm{III}}\right]$, [Ne III], $\mathrm{N}_{\mathrm{II}},\left[\mathrm{S}_{\mathrm{II}}\right],\left[\mathrm{S}_{\mathrm{III}}\right]$, and $\mathrm{He}_{\mathrm{I}}$ as well as absorption features due to the presence of $\mathrm{Zn}$ II, $\mathrm{Cr}_{\text {II, }} \mathrm{Fe}$ II, Mn II, Mg II, Mg I, Ti II, and Ca II, all at a consistent redshift. Temporal evolution of the fine-structure lines belonging to $\mathrm{Fe} \mathrm{II}^{*}$ is found between the epochs.

\section{C.12. GRB 100424A $(z=2.465)$}

The data presented here also formed the basis of GCN 14291 (Malesani et al. 2013a). The spectrum has not otherwise been published. Observations were carried out long after the burst afterglow had faded. Emission lines from the host are detected at $z=2.465$.

\section{C.13. GRB 100425A $(z=1.1755)$}

The spectra presented here also formed the basis of GCN 10684 (Goldoni et al. 2010) and are used in Skuladottir (2010), but not published elsewhere. Observations started $4 \mathrm{~h}$ after the Swift trigger, totalling $2.4 \mathrm{ks}$. Absorption features from $\mathrm{Mg}_{\mathrm{II}}$ and $\mathrm{Fe}$ II in the afterglow continuum are detected at $z=1.1755$.

\section{C.14. GRB 100615A $(z=1.398)$}

The data presented here also formed the basis of GCN 14264 (Krühler et al. 2013b), but are not published elsewhere. Host 
observation of a dark burst (D'Elia \& Stratta 2011) taken long after the afterglow had faded. Emission lines from the host belonging to [O $\mathrm{II}],[\mathrm{Ne}$ III], [O III] and $\mathrm{H} \alpha$ are detected at a common redshift of $z=1.398$.

\section{C.15. GRB 100621A $(z=0.542)$}

The data presented here also formed the basis of GCN 10876 (Milvang-Jensen et al. 2010), but are not published elsewhere. Beginning $7.1 \mathrm{~h}$ after the GRB, $2.4 \mathrm{ks}$ observations reveal emission lines from $\left[\mathrm{O}_{\mathrm{II}}\right], \mathrm{H} \beta$ and $\left[\mathrm{O}_{\mathrm{III}}\right]$ at a common redshift of $z=0.542$ and a very weak afterglow continuum.

\section{C.16. GRB 100625A $(z=0.452)$}

The data presented here is of the candidate host galaxy, taken long after the burst had faded and have not previously been published. $4.8 \mathrm{ks}$ of exposure reveals a weak continuum present in all arms, but an absence of emission lines. This could indicate that the host primarily contains an older stellar population. The redshift, $z=0.452$, is taken from Fong et al. (2013).

\section{C.17. $\operatorname{GRB} 100724 A(z=1.288)$}

The data presented here also formed the basis of GCN 10971 (Thöne et al. 2010). The spectrum has not otherwise been published previously. The observations were carried out in RRM starting $11 \mathrm{~min}$. after the GRB trigger. See Sect. 2.3, for a description of the RRM scheme. Absorption lines from several ionic species are detected in the afterglow continuum at a common redshift of $z=1.288$.

\section{C.18. GRB 100728B $(z=2.106)$}

The data presented here also formed the basis of GCN 11317 (Flores et al. 2010). The spectrum has not otherwise been published previously. Starting $22 \mathrm{~h}$ after the burst trigger, $7.2 \mathrm{ks}$ of observations reveals a faint afterglow continuum with $\operatorname{Ly} \alpha$ - and Mg II-absorption at $z=2.106$. Due to a malfunctioning ADC, the sensitivity of X-shooter is depressed with respect to normal operations, resulting in a lower throughout. Additionally, the position of the trace on the slit moves due to atmospheric differential refraction. The presence of the DLA is confirmed in the 2D image and despite the observational challenges that affects this spectrum, we measure $\log \left(N_{\mathrm{HI}} / \mathrm{cm}^{-2}\right)=21.2 \pm 0.15$.

\section{C.19. GRB 100814A $(z=1.439)$}

The spectra presented here has not been published previously. The observations consist of three visits, the first beginning only $0.9 \mathrm{~h}$ after the Swift trigger, the other two visits were 2.13 and $98.40 \mathrm{~h}$ after the trigger, respectively. A bright afterglow continuum is present in all visits, allowing identification of absorption features belonging to a wide range of ions at $z=1.439$. A complex velocity structure in the absorption features belonging to $\mathrm{Mg}$ II, shows several components, separated by as much as $500 \mathrm{~km} \mathrm{~s}^{-1}$, pointing to a likely merger scenario in the host or starburst driven outflows.

\section{C.20. GRB 100816A $(z=0.805)$}

The data presented here also formed the basis of GCN 11123 (Tanvir et al. 2010). The spectrum has not otherwise been published previously. This short GRB was observed $28.4 \mathrm{~h}$ after the GRB trigger. $4 \times 1200 \mathrm{~s}$ of exposure reveals two distinct sets of emission lines, spatially offset $\lesssim 1^{\prime \prime}$, very close in redshift space, $z=0.8034$ and $z=0.8049$, indicating either an interacting host or some complex velocity structure of the host. Faint underlying continua are present under both sets of emission lines.

\section{C.21. GRB 100901A $(z=1.408)$}

The data presented here have been published in Hartoog et al. (2013a). Because of the unusual lingering brightness of this GRB, $2.4 \mathrm{ks}$ of observations taken $65.98 \mathrm{~h}$ after the GRB trigger still reveals an afterglow continuum visible across the entire spectral coverage of X-shooter. Absorption lines from a wide range of ions sets the redshift at $z=1.408$, with intervening absorption systems at $z=1.3147$ and $z=1.3179$.

\section{C.22. GRB 101219A $(z=0.718)$}

These data have not been published before. Starting $3.7 \mathrm{~h}$ after the GRB trigger, $7.2 \mathrm{ks}$ of exposure time reveal a very faint continuum in the visual and near-infrared, only visible when heavily binning the images. No redshift estimate is available from these observations. Late-time Gemini-North observations reveal emission lines from the host at $z=0.718$ (Chornock \& Berger 2011).

\section{C.23. GRB 101219B $(z=0.552)$}

The data presented here also formed the basis of GCN 11579 (de Ugarte Postigo et al. 2011b) and are published in Sparre et al. (2011). The first observation, taken $11.6 \mathrm{~h}$ after the burst trigger and lasting $4.8 \mathrm{ks}$, reveals absorption from $\mathrm{Mg}_{\mathrm{II}}$ and $\mathrm{Mg}_{\mathrm{I}}$ in the host located at $z=0.552$ on a featureless continuum visible across the entire coverage of $\mathrm{X}$-shooter. Subsequent observations taken 16 and 37 days after the trigger shows the fading spectral signature of a SN, SN2010ma.

\section{C.24. GRB 110128A $(z=2.339)$}

These observations form the basis of GCN 11607 (Sparre et al. 2011), but have not been published before. Spectroscopic integration started $6.55 \mathrm{~h}$ after the Swift trigger and lasted for a total of $7.2 \mathrm{ks}$. The afterglow continuum is detected across the entire spectral coverage at moderate $\mathrm{S} / \mathrm{N}$. Absorption lines in the continuum are detected from Ly $\alpha, \mathrm{O}_{\text {I, }} \mathrm{C}_{\text {II }}, \mathrm{Si}$ IV, C IV, Si II and Fe II, all at a common redshift of $z=2.339$. From the broad Ly $\alpha$ trough, a hydrogen column density $\log \left(N_{\mathrm{HI}} / \mathrm{cm}^{-2}\right)=22.6 \pm$ 0.2 is derived. An intervening system at $z=2.20$ is tentatively identified from an absorption feature, likely due to $\mathrm{C}$ IV.

\section{C.25. GRB 110407A $(z=n a)$}

These observations have not been published before. Starting $12.36 \mathrm{~h}$ after the BAT trigger, $4.8 \mathrm{ks}$ spectroscopic integration yields a very faint trace down to $\sim 430 \mathrm{~nm}$, only visible after binning heavily. This could indicate a redshift, $z \sim 2.5$, but no emission lines or absorption lines are immediately visible to support this.

\section{C.26. GRB 110709B $(z=2.109)$}

This is a late-time observation ( $>1$ year) and has previously been used in Perley et al. (2016a). In this reduction of the $7.2 \mathrm{ks}$ 
spectroscopic integration, the tentative detection of [O III] reported in Perley et al. (2016a) is confirmed along with low-significance detection of $\mathrm{H} \alpha$ at the end of the spectral coverage, both at a consistent redshift, $z=2.109$, securing it as the redshift of the GRB host.

\section{C.27. GRB 110715A $(z=0.823)$}

These observations, starting $12.3 \mathrm{~h}$ after the trigger, have been published in Sánchez-Ramírez et al. (2017) and additionally formed the basis of GCN 12164 (Piranomonte et al. 2011). Only a single exposure of $600 \mathrm{~s}$ was obtained, before strong winds interrupted the observations. A red continuum is detected across all arms and a multitude of absorption lines are superposed on the afterglow continuum. We identify lines belonging to $\mathrm{Al}$ II, $\mathrm{Al}$ III, $\mathrm{Zn}$ II, $\mathrm{Cr}_{\text {II }}, \mathrm{Fe}$ II, $\mathrm{Mg}$ II, $\mathrm{Mg}_{\text {I }}, \mathrm{Ca}$ II, and $\mathrm{Ca}_{\text {II, }}$ all at $z=0.823$, marking it as the redshift of the GRB.

\section{C.28. GRB 110721A $(z=0.382)$}

This is a Fermi burst with a LAT detection and thus outside the statistical sample, but nonetheless followed up due to the extremely high peak energy (Axelsson et al. 2012). Starting $28.7 \mathrm{~h}$ after the burst trigger, $2.4 \mathrm{ks}$ spectroscopic observation reveals after heavy binning, a wide, faint trace down to $\sim 580 \mathrm{~nm}$, offset by $2.5^{\prime \prime}$ relative to the centring of the slit. No good redshift measurement can be inferred from this. We have adopted the redshift from GCN 12193 (Berger 2011).

\section{C.29. GRB 110808A $(z=1.348)$}

This spectrum has already formed the basis of GCN 12258 (de Ugarte Postigo et al. 2011c), but is not published otherwise. Starting $3 \mathrm{~h}$ after the Swift trigger, a rich spectrum is obtained in $2.4 \mathrm{ks}$ spectroscopic integration. The GRB afterglow continuum is visible across all three spectroscopic arms of VLT/Xshooter with emission lines identified as [O II], [O III], $\mathrm{H} \alpha$ all at $z=1.348$. At the same redshift, we identify absorption lines superposed on the afterglow continuum from $\mathrm{Mg}$ II and Fe II.

\section{C.30. GRB 110818A $(z=3.36)$}

Starting $6.15 \mathrm{~h}$ after the BAT trigger, spectroscopic integration for $4.8 \mathrm{ks}$ reveals a moderate $\mathrm{S} / \mathrm{N}$ GRB afterglow continuum, down to $\sim 500 \mathrm{~nm}$. The simultaneous detection of absorption features identified as Ly $\alpha, \mathrm{Si}$ II, C IV, $\mathrm{Al}$ II, Ca H, Ca K, and $\mathrm{Mg}$ II, and emission from the [O III]-doublet, securely sets $z=$ 3.36 as the redshift of the GRB. These data form the basis of GCN 12284 (D'Avanzo et al. 2011a), but is not published elsewhere.

\section{C.31. GRB 111005A $(z=0.013)$}

The data presented here have previously been published in Michałowski et al. (2018). $2.4 \mathrm{ks}$ spectroscopic integration of the host galaxy, obtained long after the burst had faded, contains bright emission lines filling the entire slit on top of a broad, underlying stellar continuum. We identify emission lines from [O II], $\mathrm{H} \delta, \mathrm{H} \gamma, \mathrm{H} \beta,\left[\mathrm{O}_{\mathrm{III}}\right], \mathrm{N}_{\mathrm{II}}, \mathrm{H} \beta$, [S II], Ar III, and [S III], all at $z=0.013$. Significant velocity structure of the lines across the spatial direction of the slit indicates a large degree of coherent motion relative to the line-of-sight.

\section{C.32. GRB 111008A $(z=4.989)$}

These data formed the basis of GCN 12431 (Wiersema et al. 2011) and are additionally published in Sparre et al. (2014). Observations of this GRB afterglow were initiated $7.71 \mathrm{~h}$ after the BAT trigger and had a duration of $8.4 \mathrm{ks}$. A second observational epoch started $20.1 \mathrm{~h}$ after the GRB trigger and lasted for $6.6 \mathrm{ks}$. The GRB afterglow continuum is well detected down to $\sim 760 \mathrm{~nm}$, with several strong absorption features imprinted, all at a common $z=4.990$. Ly $\alpha$ is clearly detected and we additionally detect lines identified as Si II, Fe II, C IV, Mg II, Si II*, $\mathrm{S}_{\text {II }}^{*}, \mathrm{O}^{*}$. An intervening DLA system is additionally detected at $z=4.61$ as seen from $\operatorname{Ly} \alpha$ and $\mathrm{Mg}$ II absorption.

\section{C.33. $G R B$ 111107A $(z=2.893)$}

GCN 12542 (D'Avanzo et al. 2011b) is based on this spectrum, but it is not published elsewhere. Spectroscopic integration started $5.26 \mathrm{~h}$ after the Swift trigger and consists of $4 \times 1200 \mathrm{~s}$ integration in the UVB and VIS and $16 \times 300 \mathrm{~s}$ in NIR, the observations ending in twilight. The GRB afterglow continuum is well detected across the arms with absorption lines from Ly $\alpha$, $\mathrm{C}_{\mathrm{IV}}, \mathrm{Fe}$ II, and Mg II, all at a consistent redshift of $z=2.893$. Additionally an intervening $\mathrm{Mg}$ II system is detected at $z=$ 1.998. From the Ly $\alpha$ absorption trough, we additionally infer $\log \left(N_{\mathrm{HI}} / \mathrm{cm}^{-2}\right)=21.0 \pm 0.2$.

\section{C.34. GRB 111117A $(z=2.211)$}

These data have previously been used to form some of the basis of Selsing et al. (2018). Starting $37.3 \mathrm{~h}$ after the BAT trigger, $4.8 \mathrm{ks}$ of spectroscopic integration yields faint emission lines identified at $\left[\mathrm{O}_{\mathrm{II}}\right], \mathrm{H} \beta,\left[\mathrm{O}_{\mathrm{III}}\right]$ and $\mathrm{H} \alpha$, all at a common $z=2.211$, marking it as the redshift of the GRB host. This is the highest redshift short GRB detected to date. The afterglow was detected in X-rays, but no optical- or radio GRB afterglow was detected.

\section{C.35. GRB 111123A $(z=3.151)$}

These data formed the basis of GCN 14273 (Xu et al. 2013a), but are not published elsewhere. Observed twice, the first time shortly after the GRB and the second long after the burst had faded, securely sets the redshift of the host at $z=3.151$ based on the detection of emission lines identified as [O II] and [O III].

\section{C.36. GRB 111129A $(z=1.080)$}

Starting $8.26 \mathrm{~h}$ after the GRB trigger and lasting $3.6 \mathrm{ks}$, these observations have previously been published in Krühler et al. (2015a). A very faint continuum is visible after severe binning and a redshift is suggested in Krühler et al. (2015a), based on the detection of [O II]. At this redshift, $\mathrm{H} \beta$ and [O II] are located in the gap between the VIS and NIR arm and $\mathrm{H} \alpha$ is located in the middle of the $J H$-band-gap and is therefore not detected.

\section{C.37. GRB 111209A $(z=0.677)$}

These spectra have previously been used in Levan et al. (2013), Greiner et al. (2015), Krühler et al. (2015a), Kann et al. (2018) and additionally formed the basis for GCN 12648 (Vreeswijk et al. 2011). The first epoch of spectroscopic 
observations was initiated $17.7 \mathrm{~h}$ after the BAT trigger and lasted for $4.8 \mathrm{ks}$. A very bright afterglow continuum is detected across the entire spectral coverage of $\mathrm{X}$-shooter, with several absorption features imprinted. The absorption features are identified as Fe II, $\mathrm{Mg}$ II, $\mathrm{Mg}$ I, $\mathrm{Ca} \mathrm{H}$, and $\mathrm{Ca} \mathrm{K}$ - all at a common redshift of $z=0.677$. The second epoch, taken 20 days later, still contains a faint continuum detected across all arms. The detection of neb-

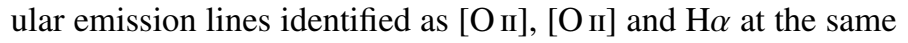
redshift, securely marks it at the redshift of this ultra-long GRB with accompanying GRB-SN.

\section{C.38. GRB 111211A $(z=0.478)$}

These data formed the basis for GCN 12677 (Vergani et al. 2011b) and are also published in Krühler et al. (2015a). Observations began $31 \mathrm{~h}$ after the AGILE trigger and consisted of $4 \times 600 \mathrm{~s}$. A bright GRB afterglow continuum is detected across the entire spectral coverage of $\mathrm{X}$-shooter with absorption and emission features visible. We identify absorption features due to Fe II, $\mathrm{Mg}$ II, and $\mathrm{Ca}$ II and emission lines from [O III] and $\mathrm{H} \alpha$, all at a common $z=0.478$, which we suggest is the redshift of the GRB. Additionally detected in the GRB afterglow continuum are broad undulation, suggesting an accompanying SN.

\section{C.39. GRB 111228A $(z=0.716)$}

These data formed the basis of GCN 12770 (Schulze et al. 2011) and are also published in Krühler et al. (2015a). Observations began $15.9 \mathrm{~h}$ after the BAT trigger and consist of $4 \times 600 \mathrm{~s}$. The GRB afterglow continuum is clearly detected in all the spectroscopic arms and superposed on the continuum are absorption features identified as due to Fe II, Mn II, $\mathrm{Mg}_{\text {II, }} \mathrm{Mg}_{\mathrm{I}}, \mathrm{Ca} \mathrm{H}$, and $\mathrm{Ca} \mathrm{K}$, all at $z=0.716$. Supporting this redshift measurement as the redshift of the GRB is the detection of nebular emission from [O III].

\section{C.40. GRB 120118B $(z=2.943)$}

The data presented here also formed the basis of GCN 14225 (Malesani et al. 2013b), but are not published otherwise. This late-time observation of the host of GRB 120118B consists of $3.6 \mathrm{ks}$ exposures and contains emission lines belonging to [O $\mathrm{II}]$ and $\left[\mathrm{O}_{\mathrm{III}}\right]$ at $z=2.943$, suggested to be redshift of the host.

\section{C.41. GRB 120119A $(z=1.728)$}

The data presented here have been examined by Japelj et al. (2015) and Zafar et al. (2018b), who both find a significant amount of extinction, $A_{V} \approx 1 \mathrm{mag}$, also supported by previous measurements (Morgan et al. 2014). Three epochs of observations have been obtained, the first two immediately after the burst, and the last one long after the afterglow had faded. Starting $1.4 \mathrm{~h}$ after the Swift trigger, the first epoch contains bright afterglow continuum. Rich in absorption features belonging to a multitude of ions at $z=1.728$ is estimated for the host with intervening systems at $z=1.476, z=1.214, z=0.662$ and $z=0.632$. The second epoch, obtained $4.5 \mathrm{~h}$ after the burst contains the fading afterglow. A third epoch is obtained $>1$ year after the GRB in which emission lines from $\mathrm{H} \beta$ and $\mathrm{H} \alpha$ are found at the redshift of the host, confirming the association of the absorption line system and the host. We also detect $\mathrm{C}_{\mathrm{I}}$ in absorption which indicates the presence of cold gas.

\section{C.42. GRB 120211A $(z=2.346)$}

The data presented here have been published in Krühler et al. (2015a). Two observations of the host of GRB 120211A has been obtained, starting 2013.02.17, >1 year after the burst has faded. A redshift for this object has been reported by Krühler et al. (2015a) and the features seen by those authors are reproduced in these reductions, confirming $z=2.346$.

\section{C.43. GRB 120224A $(z=1.10)$}

The data presented here have formed the basis of GCN 12991 (Wiersema et al. 2012b), and have also been published in Krühler et al. (2015a). Starting $19.8 \mathrm{~h}$ after the GRB trigger, a total exposure time of $2.4 \mathrm{ks}$ reveals a faint continuum, starting at $\sim 700 \mathrm{~nm}$ and extending all the way through $2500 \mathrm{~nm}$. In the $2 \mathrm{D}$-spectrum we detect a $\sim 2 \sigma$ emission line which, if interpreted as $\mathrm{H} \alpha$, gives $z=1.10$, supporting the photometric redshift $\left(0.9<z_{\text {phot }}<1.3\right)$ derived by Krühler et al. (2015a).

\section{C.44. GRB $120311(z=0.350)$}

The data presented here have formed the basis of GCN 12991 (Wiersema et al. 2012b), but are not published otherwise. Starting just before twilight, $3.65 \mathrm{~h}$ after the burst, a faint afterglow continuum is detected at all wavelengths. Due to the faintness of the afterglow, no absorption features are discernible superposed on the continuum. Displaced from the afterglow continuum by 1 '. 4 , emission lines belonging to $\mathrm{H} \beta$, [O III] and $\mathrm{H} \alpha$ are detected at $z=0.350$. The line belonging to $\mathrm{H} \alpha$ shows some extended emission towards the afterglow continuum. The angular distance between the two sources correspond to a projected distance in the host plane of $6 \mathrm{kpc}$, posing a potential problem for the host redshift, unless the GRB occurred in a merging system. The extended emission in $\mathrm{H} \alpha$, supports this interpretation. This burst is not a part of the statistical sample.

\section{C.45. GRB 120327A $(z=2.813)$}

The data presented here also formed the basis of GCN 13134 (Krühler et al. 2012b) and are published in D'Elia et al. (2014). The observation consists of two visits, $2.13 \mathrm{~h}$ and $29.98 \mathrm{~h}$ after the burst, with an afterglow continuum visible in all arms for both visits. We detect absorption features from Ly-limit, Ly $\alpha, \mathrm{C}_{\text {II }} / \mathrm{C}_{\text {I }}{ }^{*}$, $\mathrm{Si}$ II/Si II ${ }^{*}, \mathrm{Al}$ I, Fe II and $\mathrm{Mg}$ II at a consistent redshift, $z=2.813$.

\section{C.46. GRB 120404A $(z=2.876)$}

The data presented here have formed the basis of GCN 13227 (D'Elia et al. 2012a), but are not published otherwise. 9.6 ks integration, starting $15.7 \mathrm{~h}$ after the Swift-trigger reveals a lowintensity afterglow continuum on which absorption from Ly $\alpha$ is detected in two distinct regions at redshifts $z=2.876$ and $z=2.55$. These absorption systems are confirmed by ionic absorption features at both of these redshifts.

\section{C.47. $G R B$ 120422A $(z=0.283)$}

The data presented here also formed the basis of GCN 13257 (Schulze et al. 2012) and are published in Schulze et al. (2014a). Being a GRB-SN, this burst has been followed up multiple times. The data presented here only contain the first epoch in which the afterglow is still visible and before the rise of SN2012bz. 
Starting $16.5 \mathrm{~h}$ after the burst, $4.8 \mathrm{ks}$ integration time captures both the host and the burst in emission. A blue afterglow continuum is detected at all wavelengths covered by $\mathrm{X}$-shooter, on which $\mathrm{Mg}$ II absorption at $z=0.283$ is found. Offset by 1 '. 75 , the host is clearly detected at a consistent redshift with a rich emission line spectrum, the lines extending towards to burst.

\section{C.48. GRB 120712A $(z=4.175)$}

The data presented here also formed the basis of GCN 13460 (Xu et al. 2012), but are not published elsewhere. $4.8 \mathrm{ks}$ integration time, starting $10.5 \mathrm{~h}$ after the BAT trigger, shows a bright afterglow continuum starting at $\sim 472 \mathrm{~nm}$, signifying the onset of the Lyman alpha forest, for a GRB located at $z=4.175$. Absorption features from $\mathrm{Ly} \alpha, \mathrm{Fe}$ II, $\mathrm{Mg}$ II and $\mathrm{Si}_{\text {II }}$ are readily detected at a consistent redshift.

\section{C.49. GRB 120714B $(z=0.398)$}

The data presented here also formed the basis of GCN 13477 (Fynbo et al. 2012a) and are discussed in Klose et al. (2019), but are not published anywhere. Observations of this burst started $7.8 \mathrm{~h}$ after the GRB trigger, lasting $4.8 \mathrm{ks}$. A continuum is visible across the entire spectral coverage of X-shooter, with both emission lines from [O II], $\mathrm{H} \beta$, [O III] and $\mathrm{H} \alpha$, as well as absorption from $\mathrm{Mg}$ II detected at $z=0.398$, securely setting it as the redshift of the GRB.

\section{C.50. GRB 120716A $(z=2.486)$}

The data presented here also formed the basis of GCN 13494 (D'Elia et al. 2012b), but are not published elsewhere. Despite observations starting $62 \mathrm{~h}$ after the Swift trigger and lasting $3.6 \mathrm{ks}$, a bright afterglow is clearly seen, along with a plethora of absorption features. Absorption of $\operatorname{Ly} \alpha$-photons in the host leaves a broad trough, beyond which the Lyman alpha forest is visible bluewards, all the way down to the Lyman limit. Metal absorption lines from $\mathrm{C}_{\text {II, }}$ Si II, O I, Fe II, C IV, Si IV, including fine-structure transitions identified as $\mathrm{C}_{\text {II }}{ }^{*}, \mathrm{Si}_{\text {II }}{ }^{*}, \mathrm{Fe}$ II $^{*}$ and metastable Ni II lines are all detected at $z=2.486$

\section{C.51. GRB 120722A $(z=0.959)$}

The data presented here also formed the basis of GCN 13507 (D'Elia et al. 2012c), but are not published elsewhere. In $4.8 \mathrm{ks}$ integration time, starting $10 \mathrm{~h}$ after the burst trigger, the simultaneous detection of absorption features belonging to $\mathrm{Mg}$ II and Fe II superposed on a blue continuum, and emission lines from [O II], $\mathrm{H} \gamma, \mathrm{H} \beta,[\mathrm{O}$ III] and $\mathrm{H} \alpha$, all at $z=0.959$, confidently sets it as the redshift of the GRB.

\section{C.52. GRB 120805A $(z=3.9)$}

A separate reduction of this burst has been published in Krühler et al. (2015a), but is not used otherwise. Starting 9 days after the burst trigger, this is a host observation and does not contain any afterglow continuum. In $3.6 \mathrm{ks}$ integration time, we detect a faint continuum visible from $450 \mathrm{~nm}$ and all the way through $2100 \mathrm{~nm}$, in contrast to what is found previously. The continuum from 450$600 \mathrm{~nm}$ is detected at very low significance. If the drop at $450 \mathrm{~nm}$ is the Lyman limit, this fits with Lyman alpha at $\sim 600 \mathrm{~nm}$, giving $z \sim 3.9$. The absence of nebular lines is due to [O II] falling in a telluric absorption band and the rest of the strong nebular lines being shifted out of the wavelength coverage.

\section{C.53. GRB 120815A $(z=2.358)$}

Not a part of the statistical sample, this burst also formed the basis of GCN 13649 (Malesani et al. 2012) and is published in Krühler et al. (2013a). Observations started $1.69 \mathrm{~h}$ after the BAT trigger and consist of $2.4 \mathrm{ks}$ integration. A bright afterglow continuum is detected across the entire spectral coverage of X-shooter, with a multitude of absorption lines superposed. Absorption features from the host at $z=2.358$ include a DLA as well as metal absorption lines from $\mathrm{Nv}, \mathrm{S}$ II, Si II, O I, C IV, Si IV, Fe II, Al II, Al III, Mn II, Mg II, and Mg I. Also fine-structure lines from $\mathrm{Ni}$ II and $\mathrm{Fe}$ II are excited in the local environment of the GRB. Additionally, this spectrum is one of the rare cases in which we detect lines from molecular hydrogen, $\mathrm{H}_{2}$. From the Ly $\alpha$-line we measure $\log \left(N_{\mathrm{HI}} / \mathrm{cm}^{-2}\right)=22.1 \pm 0.10$. Intervening systems are found at $z=1.539, z=1.693$, and $z=2.00$.

\section{C.54. GRB 120909A $(z=3.929)$}

The data presented here have formed the basis of GCN 13730 (Hartoog et al. 2012), but are not published otherwise. Followup, started only $1.7 \mathrm{~h}$ after the BAT trigger. This $1.2 \mathrm{ks}$ observation captures a very bright afterglow continuum, starting at $450 \mathrm{~nm}$, signifying the onset of the Lyman limit for a system at $z=3.929$. Absorption from high-column density hydrogen leaves very prominent absorption features in the form of Ly $\alpha$, $\operatorname{Ly} \beta$, and $\operatorname{Ly} \gamma$, visible in the Lyman alpha forest. Metal absorption lines arising from $\mathrm{Fe}_{\text {II, }} \mathrm{Ni}$ II, $\mathrm{Si}$ II, $\mathrm{S}_{\text {II, }} \mathrm{Al}$ II, $\mathrm{Al}_{\text {III, }} \mathrm{C}_{\text {II, }} \mathrm{O}_{\text {I, }}$ $\mathrm{C}_{\mathrm{IV}}$, and $\mathrm{Zn}$ II are all detected along with the corresponding finestructure lines (Fe II $\left.{ }^{*}, \mathrm{Si} \mathrm{II}^{*}, \mathrm{O}^{*}, \mathrm{O} \mathrm{I}^{*} *, \mathrm{C}_{\mathrm{II}^{*}}\right)$, securely anchoring the redshift of the host.

\section{C.55. GRB 120923A $(z=7.84)$}

This spectrum has previously been published in Tanvir et al. (2018). Starting $18.5 \mathrm{~h}$ after the BAT trigger, the final spectrum is based on $2 \times 4 \times 1200 \mathrm{~s}$ spectroscopic integration for a total of $160 \mathrm{~min}$. Nothing is immediately visible in the 2D-spectra, however after severe binning in the dispersion direction, a faint trace shows up in the NIR arm, suggesting a very high redshift. We here adopt the redshift $(z=7.84)$ suggested by Tanvir et al. (2018).

\section{C.56. GRB 121024A $(z=2.300)$}

The data presented here also formed the basis of GCN 13890 (Tanvir et al. 2012) and are published in Friis et al. (2015). Starting $1.8 \mathrm{~h}$ after the Swift trigger, a bright afterglow continuum is visible across all arms. A broad absorption feature from Lyman alpha, along with narrow lines from $\mathrm{C}_{\text {IV }}, \mathrm{Si}$ II, Si IV, Fe II, S II, and $\mathrm{Al}$ II, as well as fine-structure lines associated with $\mathrm{Si} \mathrm{II}^{*}$ are all detected at $z=2.300$, securely setting it as the redshift of the GRB.

\section{C.57. GRB 121027A $(z=1.773)$}

The data presented here have formed the basis of GCN 13930 (Krühler et al. 2012c), but are not published otherwise. Starting 69.6 h after the GRB trigger, we detect the afterglow continuum at high significance in all arms with $8.4 \mathrm{ks}$ integration, testifying to the brightness of this burst. The concurrent identification of emission lines from [O III] and absorption from $\mathrm{C}$ IV, $\mathrm{Al}$ II, Al III, $\mathrm{Mg}_{\mathrm{I}}, \mathrm{Mg}$ II, and Fe II, tightly constrains the redshift of the burst to be $z=1.773$. 


\section{C.58. GRB 121201A $(z=3.385)$}

These data formed the basis for GCN 14035 (Sanchez-Ramirez et al. 2012) and are additionally published in Krühler et al. (2015a). These observations started $12.9 \mathrm{~h}$ after the Swift trigger and consist of $4.8 \mathrm{ks}$ spectroscopic integration under good atmospheric conditions. The GRB afterglow continuum is well detected at all arms. A broad absorption trough due to Ly $\alpha$ is visible at $z=3.385$, which along with the detection of absorption features identified as $\mathrm{Si}$ IV, $\mathrm{C}$ IV, $\mathrm{Al}$ II, and $\mathrm{Al}$ III, marks it as the redshift of the GRB. In the middle of the Ly $\alpha$ trough, we additionally detect $\operatorname{Ly} \alpha$ emission. By modelling the Ly $\alpha$ absorption, we infer $\log \left(N_{\mathrm{HI}} / \mathrm{cm}^{-2}\right)=22.0 \pm 0.3$.

\section{C.59. $\operatorname{GRB} 121229 A(z=2.707)$}

These data formed the basis for GCN 14120 (Fynbo et al. $2012 \mathrm{~b}$ ), but are not published elsewhere. Taken under poor seeing conditions, a total of $4.8 \mathrm{ks}$ spectroscopic integration starting $2 \mathrm{~h}$ after the $S$ wift trigger yields a low S/N GRB afterglow continuum is all arms. Binning the spectrum reveals broad absorption troughs, which we identify as $\operatorname{Ly} \beta$ and $\operatorname{Ly} \alpha$ at $z=2.707$. Additionally, an intervening system at $z=1.658$ is detected from absorption features of $\mathrm{Mg}$ II. From the absorption trough due to Ly $\alpha$, we infer $\log \left(N_{\mathrm{HI}} / \mathrm{cm}^{-2}\right)=21.7 \pm 0.2$. Due to strong contamination in the slit, the background is slightly over subtracted, causing the background to be negative in the centre of the Ly $\alpha$ trough.

\section{C.60. GRB 130131B $(z=2.539)$}

These data formed the basis for GCN 14286 (Fynbo et al. 2013) and are additionally published in Krühler et al. (2015a). This is a late-time observation, taken long after the GRB afterglow had faded. In $7.2 \mathrm{ks}$ spectroscopic integration, emission lines identified as $\left[\mathrm{O}_{\mathrm{II}}\right]$ and $\left[\mathrm{O}_{\mathrm{III}}\right]$ are detected at a common $z=2.539$, which we suggest is the redshift of the GRB.

\section{C.61. GRB 130408A $(z=3.758)$}

The data presented here also formed the basis of GCN 14365 (Hjorth et al. 2013). The spectrum has not otherwise been published previously. The observations consists of two $600 \mathrm{~s}$ spectra taken $1.9 \mathrm{~h}$ after the burst. We detect absorption features from a wide range of ions. We also detect intervening absorption at $z=1.255$ and $z=3.248$.

\section{C.62. GRB $130418 A(z=1.222)$}

GCN 14390 (Krühler et al. 2013c) is based on this spectrum, but it is not published elsewhere. Starting only $4.57 \mathrm{~h}$ after the Swift trigger, $1.2 \mathrm{ks}$ observations contain a bright GRB afterglow continuum, visible across the entire spectral coverage of X-shooter. Superposed on the afterglow continuum are absorption features which we identify as $\mathrm{C}_{\mathrm{IV}}, \mathrm{Fe}$ II, and $\mathrm{Mg}$ II, caused by an absorber at $z=1.217$, and additional absorption from $\mathrm{C}_{\mathrm{IV}}$ at $z=1.222$. The two systems are offset by $\sim 1500 \mathrm{~km} \mathrm{~s}^{-1}$ and the proximity of the two absorption systems in velocity space, suggests a possible association of the two systems with peculiar velocity affecting the measured redshift. We adopt $z=1.222$ as the redshift of the GRB. Note, that this value is slightly different from the one reported in GCN 14390.

\section{C.63. GRB 130427A $(z=0.340)$}

This spectrum is also published in $\mathrm{Xu}$ et al. (2013b) and Krühler et al. (2015a) and additionally has formed the basis for GCN 14491 (Flores et al. 2013a). Starting 16.5 h after the BAT trigger, these observations lasting $2 \times 600 \mathrm{~s}$ contain a very bright GRB afterglow continuum across the total spectral coverage of $\mathrm{X}$-shooter. In absorption we identify features from the following metal resonance lines: Fe II, $\mathrm{Mn}$ II, $\mathrm{Mg}$ II, $\mathrm{Mg}$ I, Ti II and additional line absorption from $\mathrm{Ca}$ II and Na ID. Simultaneously, we find emission lines from $\mathrm{H} \alpha, \mathrm{H} \beta$, [O III], [O II]- all at common redshift of $z=0.340$, which is the redshift of the GRB. This is one of the most energetic GRBs observed, and its proximity along with the associated broad-lined Type Ic SN, 2013cq has caused it to be one of the more well-studied GRBs (Maselli et al. 2014; Perley et al. 2014; Ackermann et al. 2014).

\section{C.64. GRB 130427B $(z=2.780)$}

This spectrum formed the basis of GCN 14493 (Flores et al. $2013 \mathrm{~b}$ ), but are not published otherwise. A short, $2 \times 600 \mathrm{~s} \mathrm{spec-}$ troscopic integration obtained before twilight, $20.6 \mathrm{~h}$ after the BAT trigger, captures a faint afterglow continuum visible across the entire spectral coverage at low $\mathrm{S} / \mathrm{N}$. Due to the low $\mathrm{S} / \mathrm{N}$, the metal lines are weak, but the broad absorption trough due to Ly $\alpha$ is detected. From the Ly $\alpha$ line we measure the redshift to be $z=2.780$ and provide a measure of the neutral hydrogen column density, $\log \left(N_{\mathrm{HI}} / \mathrm{cm}^{-2}\right)=21.9 \pm 0.3$. The redshift is confirmed by the presence of $\mathrm{Fe}$ II absorption at a consistent redshift.

\section{C.65. GRB 130603B $(z=0.356)$}

This burst is the first short GRB observed with a potential associated kilonova (Tanvir et al. 2013a; Berger et al. 2013). GCN 14757 (Xu et al. 2013c) was based on this spectrum and it is additionally published in de Ugarte Postigo et al. (2014b). Starting $8.2 \mathrm{~h}$ after the Swift trigger, a total of $2.4 \mathrm{ks}$ spectroscopic integration was obtained. Spectral continuum is clearly detected across all arms from both host and afterglow and superposed are both absorption ( $\mathrm{Ca} \mathrm{H} \& \mathrm{~K}$ and $\mathrm{Mg}$ II) and emission lines ([O II] $, \mathrm{H} \beta,\left[\mathrm{O}_{\mathrm{III}}\right], \mathrm{H} \alpha$, and [S $\left.\mathrm{II}\right]$ ), all at a consistent redshift of $z=0.356$, which is the redshift of the GRB.

\section{C.66. GRB 130606A $(z=5.913)$}

The data presented here also formed the basis of GCN 14816 (Xu et al. 2013d) and are published in Hartoog et al. (2015). The observations consist of three $2 \times 600 \mathrm{~s}$ visits starting $7.1 \mathrm{~h}$ after the burst at fairly high airmass. We detect absorption features from a wide range of ions at $z=5.913$ as well as intervening absorption at $z=2.3103,2.5207,3.4515,4.4660,4.5309,4.5427,4.6497$ and 4.7244 .

\section{C.67. GRB 130612A $(z=2.006)$}

The spectral features of this spectrum have previously been reported in GCN 14882 (Tanvir et al. 2013b), but are not published elsewhere. Starting only $1.1 \mathrm{~h}$ after the Swift trigger, $2 \times 600$ s spectroscopic integration captures a moderate $\mathrm{S} / \mathrm{N}$ afterglow continuum across the total spectral coverage of $\mathrm{X}$-shooter. At a consistent redshift of $z=2.006$, absorption from the metal resonance lines Fe II, $\mathrm{Mn}$ II, $\mathrm{Mg}$ II, $\mathrm{Mg}$ I are identified. Additionally, Ly $\alpha$ is visible as a broad absorption trough, from which we 
can infer $\log \left(N_{\mathrm{HI}} / \mathrm{cm}^{-2}\right)=22.2 \pm 0.2$, which is in the upper end of the hydrogen column density distribution. The blue part of the GRB continuum exhibits a downturn in the continuum level which could indicate the presence of a significant amount of dust along the line-of-sight.

\section{C.68. GRB 130615A $(z=2.9)$}

This spectrum has not previously been published. Starting only $45 \mathrm{~min}$ after the BAT trigger, $2 \times 600 \mathrm{~s}$ spectroscopic integration carried on into the beginning twilight. Observed at very high airmass with a quickly varying background, a faint afterglow trace is visible across all arms of X-shooter, down to $480 \mathrm{~nm}$, which if interpreted as the break due to Ly $\alpha$ suggests $z=2.9$. This supports the approximate redshift suggested in GCN 14898 (Elliott et al. 2013).

\section{C.69. GRB 130701A $(z=1.155)$}

These data formed the basis for GCN 14956 (Xu et al. 2013e) and are additionally published in Krühler et al. (2015a). Starting $5.5 \mathrm{~h}$ after the GRB trigger, $2 \times 600 \mathrm{~s}$ reveals a bright continuum visible across the entire spectral coverage of $\mathrm{X}$-shooter. Superposed are absorption features which we identify as due to Fe II, $\mathrm{Mg}$ II, $\mathrm{Mg}$ I, and Ca II- all at a consistent redshift of $z=1.155$, which we take to be the redshift of the GRB.

\section{C.70. GRB 130925A $(z=0.347)$}

This spectrum has already been used in GCN 15250 (Sudilovsky et al. 2013) and is additionally published in Schady et al. (2015) and Krühler et al. (2015a). Observations of this burst began with $\mathrm{X}$-shooter $3.5 \mathrm{~h}$ after the $S$ wift trigger. $6 \mathrm{ks}$ spectroscopic integration captures a heavily dust obscured afterglow $\left(A_{V}=5.9 \pm 0.7\right.$; Greiner et al. 2014), with the spectrum primarily dominated by host emission lines. All the nebular lines ([O II], $\mathrm{H} \gamma, \mathrm{H} \beta$, [O II], $\left.\mathrm{H} \alpha, \mathrm{N}_{\text {III }}\left[\mathrm{S}_{\mathrm{II}}\right]\right)$ are well detected at $z=0.347$, as well as those from $\mathrm{Pa} \delta, \operatorname{Pa} \gamma$, and $\mathrm{Pa} \beta$. We take this as the redshift of the GRB. This spectrum is taken under ESO programme ID: 091.A0877(A) (PI: Schady).

\section{C.71. GRB 131011A $(z=1.874)$}

These data formed the basis for GCN 15330 (Rau et al. 2013), but are not published elsewhere. Starting $\sim 1.5$ days after the Fermi-GBM trigger, $4.5 \mathrm{ks}$ spectroscopic integration captures a modest $\mathrm{S} / \mathrm{N}$ GRB afterglow continuum all the way down to $\sim 320 \mathrm{~nm}$. Imprinted on the continuum are absorption features, which we identify as due to $\operatorname{Ly} \alpha, \mathrm{Fe}$ II, $\mathrm{Mg}_{\text {II, }} \mathrm{Mg}_{\mathrm{I}}$ at the same redshift, which we measure to be $z=1.874$. From the broad absorption trough due to Ly $\alpha$, we infer $\log \left(N_{\mathrm{HI}} / \mathrm{cm}^{-2}\right)=22.0 \pm$ 0.3. This spectrum is taken under ESO programme ID: 092.D0056(A) (PI: Rau).

\section{C.72. GRB 131030A $(z=1.296)$}

These data has not been published before. Starting $3.4 \mathrm{~h}$ after the Swift trigger, $6 \times 600 \mathrm{~s}$ exposure were taken under good conditions, containing a bright GRB afterglow continuum across the entire spectral coverage of X-shooter. A myriad of absorption features are superposed on the afterglow continuum which we identify as being caused by Si Iv, Si II, C IV, Al II, Al III, Zn II, Cr II, Ni II, Fe II, Ni II*, and Fe II* at $z=1.296$. A very strong
$\mathrm{Mg}$ II-absorber is also detected, intervening the line-of-sight at $z=1.164$ with lines from Si II, C IV, Al III, Al III, Fe II, Mn II, and many more.

\section{C.73. GRB 131103A $(z=0.599)$}

This spectrum has already been used to form the basis for GCN 15451 (Xu et al. 2013f) and is additionally published in Krühler et al. (2015a). Starting $5.8 \mathrm{~h}$ after the BAT trigger, $4 \times 600 \mathrm{~s}$ exposure captures a modest $\mathrm{S} / \mathrm{N}$ continuum across all arms. Imprinted on the continuum are absorption features identified as due to $\mathrm{Fe}$ II and $\mathrm{Mg}$ II as well as emission lines from [O II], $\mathrm{H} \delta, \mathrm{H} \gamma, \mathrm{H} \beta,[\mathrm{O}$ III], $\mathrm{H} \alpha$, and [N $\mathrm{NI}$. All the lines are measured at a consistent redshift of $z=0.599$, which we take as the redshift of the GRB.

\section{C.74. GRB 131105A $(z=1.686)$}

This spectrum has already been used in GCN 15450 (Xu et al. 2013g) and is additionally published in Krühler et al. (2015a). Starting only $1.3 \mathrm{~h}$ after the Swift trigger, a total of $4.8 \mathrm{ks}$ spectroscopic integration contains a low $\mathrm{S} / \mathrm{N}$ GRB afterglow continuum across the entire spectral coverage of X-shooter. There are deviations from the continuum at both emission and absorption. We identify lines from $\mathrm{H} \beta$, [O III], and $\mathrm{H} \alpha$ in emission and Fe II, and $\mathrm{Mg}$ II in absorption. All lines are at a consistent $z=1.686$, which is probably the redshift of the GRB. Absorption lines at shorter wavelengths are also detected, but at low significance due to an apparent downturn in the continuum caused by the presence of dust local to the burst.

\section{C.75. GRB 131117A $(z=4.042)$}

This spectrum has previously been used in GCN 15494 (Hartoog et al. 2013b), but is not published in a refereed paper. Starting only $68 \mathrm{~min}$ after the BAT trigger, $4.8 \mathrm{ks}$ spectroscopic integration secures afterglow continuum for this burst, which is measured to be at $z=4.042$. A moderate S/N GRB afterglow continuum is detected down to $\sim 610 \mathrm{~nm}$, signifying the onset of the Ly $\alpha$ forest, with part of the forest also visible. Metal absorption lines from $\mathrm{Si}$ II and $\mathrm{Si}$ IV are detected at a consistent redshift.

\section{C.76. GRB 131231A $(z=0.642)$}

This spectrum has previously been published in Krühler et al. (2015a) and additionally forms the basis for GCN 15645 (Xu et al. 2014a). This spectrum, observed the following year $(20.2 \mathrm{~h}$ after the Swift trigger), consists of $4 \times 600$ s exposures. A high $\mathrm{S} / \mathrm{N}$ GRB afterglow continuum is detected all the way through the X-shooter arms. We identify absorption features imprinted in the continuum as caused by $\mathrm{Fe}_{\mathrm{II}}, \mathrm{Mg}$ II, and $\mathrm{Ca} \mathrm{H} \mathrm{\&} \mathrm{K}$ at a consistent $z=0.642$. By subtracting off the bright afterglow continuum, we readily detect emission lines arising from [O II], $\mathrm{H} \gamma, \mathrm{H} \beta$, [O III], and $\mathrm{H} \alpha$ in the GRB host galaxy. This GRB is the subject of a forthcoming work Kann et al. (in prep.).

\section{C.77. GRB 140114A $(z=3.0)$}

This spectrum has previously been published in Krühler et al. (2015a). This is a late time observation, taken long after the GRB had faded. Despite a long integration time of $5.4 \mathrm{ks}$, no clear features stand out to clearly secure a redshift measurement. By heavily binning the spectrum, a faint trace is visible down to 
$485 \mathrm{~nm}$, which if interpreted at the onset of Ly $\alpha$ signifies $z \sim 3$. We adopt the redshift inferred in Krühler et al. (2015a).

\section{C.78. GRB 140213A $(z=1.208)$}

The data presented here also formed the basis of GCN 15831 (Schulze et al. 2014b) and are additionally published in Krühler et al. (2015a). Starting 5.8 after the Swift trigger alert, $2 \times 600 \mathrm{~s}$ spectroscopic integration contains a high S/N GRB afterglow continuum across the entirety of $\mathrm{X}$-shooter. Imprinted on the afterglow continuum are absorption features, which we identify as metal resonance lines from $\mathrm{C}_{\mathrm{IV}}, \mathrm{Al}$ II, $\mathrm{Al}$ III, Fe II, $\mathrm{Mg}_{\text {II }}$, and $\mathrm{Mg}$ I. These lines are likely formed by metals in the GRB host, which we measure to be at $z=1.208$.

\section{C.79. GRB 140301A $(z=1.416)$}

These data formed the basis for GCN 15900 (Krühler et al. 2014a) and are additionally published in Krühler et al. (2015a). Spectroscopic follow-up began $9 \mathrm{~h}$ after the BAT trigger and lasted for $12 \times 600 \mathrm{~s}$. A low S/N, spatially extended continuum is visible across the entire spectral coverage of $\mathrm{X}$-shooter. The GRB afterglow continuum is visible at moderate $\mathrm{S} / \mathrm{N}$ on top of the underlying host continuum. The host exhibits usual nebular emission from [O II], $\mathrm{H} \beta$, [O III], $\mathrm{H} \alpha, \mathrm{N}_{\text {III }}$, and [S II] which puts it at $z=1.416$. Supporting this as the redshift of the GRB are absorption features from the $\mathrm{Mg}$ II-doublet in the GRB afterglow continuum.

\section{C.80. GRB 140311A $(z=4.954)$}

This spectrum has not been published previously. Starting $32.5 \mathrm{~h}$ after the GRB trigger on-board Swift, this observation lasted $14 \times 600 \mathrm{~s}$ for a total of $8.4 \mathrm{ks}$. Some loss occurred during the observations, which reduced integration time in the UVB and VIS arm slightly. The GRB afterglow continuum is clearly visible in the VIS and NIR arm of X-shooter. The continuum is very rich in absorption features, with at least the following lines identified: Ly $\gamma, \mathrm{Ly} \beta, \mathrm{Ly} \alpha, \mathrm{Si}$ II, Si Iv, C IV, Al II, Al III, Fe II, Mg II, and $\mathrm{Mg}$ I. All of these lines are at $z=4.954$, which we take as the redshift of the GRB. This spectrum is taken under ESO programme ID: 092.D-0633(E) (PI: Greiner).

\section{C.81. GRB 140430A $(z=1.601)$}

A separate reduction of this burst has been published in Krühler et al. (2015a), and additionally the spectrum has also been used in GCN 16194 (Krühler et al. 2014b). Observations for this burst began in twilight, $2.5 \mathrm{~h}$ after the BAT trigger and lasted for $2 \times 600 \mathrm{~s}$. The spectrum contains a moderate S/N GRB afterglow continuum all the way through the spectroscopic arms of X-shooter. We identify absorption features in the afterglow continuum from Si II, C IV, $\mathrm{Al}$ II, Fe II, and $\mathrm{Mg}_{\text {II, }}$ and emission lines from [O II] and [O III] - all at $z=1.601$ which is likely the redshift of the GRB.

\section{C.82. GRB 140506A $(z=0.889)$}

The data presented here has formed the basis of GCN 16217 (Fynbo et al. 2014a) and is published in Fynbo et al. (2014b), Krühler et al. (2015a), and Heintz et al. (2017). The observations consists of $4 \times 600 \mathrm{~s}$ at 8.8 and $33 \mathrm{~h}$ after the burst. We detect absorption features from a wide range of ions, together with molecular absorption from $\mathrm{CH}+$, all at $z=0.889$. The optical/near-infrared afterglow reveals an unusual steep extinction curve which is found to be caused by dust very close to the burst.

\section{C.83. GRB 140515A $(z=6.327)$}

This spectrum has previously been used in Melandri et al. (2015). Starting $15.5 \mathrm{~h}$ after the Swift trigger, $8 \times 600 \mathrm{~s}$ spectroscopic integration captures this very high redshift GRB afterglow. The Gunn-Peterson trough is visible against the GRB afterglow continuum, starting at $890 \mathrm{~nm}$, which along with absorption lines from the $\mathrm{Mg}$ II doublet securely sets the redshift of this GRB at $z=6.327$. From the red wing of the Ly $\alpha$-profile we measure $\log \left(N_{\mathrm{HI}} / \mathrm{cm}^{-2}\right)=19.0 \pm 0.5$, which is very low compared to the measured distribution of $N_{\mathrm{HI}}$.

\section{C.84. GRB 140614A $(z=4.233)$}

This spectrum forms the basis for GCN 16401 (Krühler et al. 2014c), but is not published elsewhere. $4 \times 600 \mathrm{~s}$ spectroscopic integration, starting $3.8 \mathrm{~h}$ after the BAT trigger catches this GRB afterglow, which turns out to be at very high redshift. The continuum is detected at moderate $\mathrm{S} / \mathrm{N}$ in both the VIS and NIR arms of $\mathrm{X}$-shooter, heavily affected by absorption features. We identify the lines belonging to $\mathrm{Ly} \alpha, \mathrm{Si}$ II, $\mathrm{C}_{\text {II, }} \mathrm{C}_{\text {II }}{ }^{*}, \mathrm{Al}_{\text {II, }} \mathrm{Al}$ III, Fe II, and $\mathrm{Mg}$ II at a consistent redshift of $z=4.233$. From the shape of the Ly $\alpha$ absorption trough we measure $\log \left(N_{\mathrm{HI}} / \mathrm{cm}^{-2}\right)=21.3 \pm 0.3$.

\section{C.85. GRB 140622A $(z=0.959)$}

The characteristics of this short $(\mathrm{T} 90=0.13 \pm 0.04 \mathrm{~s}$; Lien et al. 2016) GRB spectrum has been published in GCN 16437 (Hartoog et al. 2014), but does not appear in the refereed literature. Spectroscopic observations began with $\mathrm{X}$-shooter only $34 \mathrm{~min}$ after the BAT trigger and lasted for $2 \times 600 \mathrm{~s}$. In the spectrum, we detect continuum across all three arms of $\mathrm{X}$-shooter. In the UVB arm, the continuum is only visible after heavily binning the spectrum in the dispersion direction. It is unclear how much of the continuum is from the host galaxy and how much is from the potential GRB afterglow. Superposed on the continuum, emission lines from [O II] $, \mathrm{H} \beta,\left[\mathrm{O}_{\mathrm{III}}\right], \mathrm{H} \alpha$ are all detected at $z=0.959$. We use this as the likely redshift of the GRB.

\section{C.86. GRB 141028A $(z=2.332)$}

This spectrum has already been used in GCN 16983 (Xu et al. 2014b), but is not published elsewhere. Starting $15.4 \mathrm{~h}$ after the Swift trigger and lasting for a total of $2.4 \mathrm{ks}$, these observations captures a bright GRB afterglow. Very rich in absorption, the continuum is detected across the entire spectral coverage of $\mathrm{X}$-shooter, except the blue half of the UVB arm, where the Ly $\alpha$ forest absorbs the continuum. The redshift of the host of the GRB is measured based in the detection of features from Ly $\alpha$, Si II, C IV, C II, Fe II, and Mg II - all at a consistent $z=2.332$. From the Ly $\alpha$ trough we infer $\log \left(N_{\mathrm{HI}} / \mathrm{cm}^{-2}\right)=20.6 \pm 0.15$. Two intervening system at $z=1.823$ and $z=2.09$ are also found in the spectrum based on the detection of C IV.

\section{C.87. GRB 141031A $(z=n a)$}

This spectrum is an attempt at a late-time host redshift measurement for GRB 141031A. The spectrum is taken long after the 
burst has faded and consists of $4 \times 600$ s spectroscopic integration. In the $2 \mathrm{D}$ spectrum are two sources, which are both offset from the targeted host position and are thus likely foreground objects. The spectrum does not contain anything that can be used to measure a redshift from.

\section{C.88. GRB 141109A $(z=2.993)$}

The gross content of the spectrum has been issued in GCN 17040 (Xu et al. 2014c), but it is not used otherwise. Starting only $1.9 \mathrm{~h}$ after the BAT trigger, these $4 \times 600 \mathrm{~s}$ spectra contain a high $\mathrm{S} / \mathrm{N}$ GRB afterglow. The afterglow continuum is readily detected all the way down to $370 \mathrm{~nm}$, although the bluest part is affected by the Ly $\alpha$-forest. A broad absorption trough from neutral hydrogen is clearly visible, with additional, narrower absorption features identified as due to $\mathrm{Si}$ II, Si II ${ }^{*}, \mathrm{C}_{\text {II, }} \mathrm{C}_{\text {II }^{*}}, \mathrm{Si}$ IV, C IV, Fe II, Fe II*, $\mathrm{O}^{*}$, and $\mathrm{N}_{\mathrm{II}}{ }^{*}$. These are all detected at a consistent $z=2.993$, which due to the detection of the locally excited fine-structure lines securely sets this as the redshift of the GRB. Additionally, two $\mathrm{Mg}$ II absorption systems are detected at $z=1.67$ and $z=2.5$. From the broad $\operatorname{Ly} \alpha$-absorption, we measure a neutral hydrogen column density of $\log \left(N_{\mathrm{HI}} / \mathrm{cm}^{-2}\right)=22.1 \pm 0.1$.

\section{C.89. GRB 150206A $(z=2.087)$}

This spectrum has previously been used to form the basis for GCN 17420 (Krühler et al. 2015b), but it is not published in the refereed literature. Due to the short visibility of this burst, it does not enter into the statistical sample. Beginning in twilight, $4 \times 600 \mathrm{~s}$ integration time contains a weak/moderate S/N GRB afterglow continuum throughout the $\mathrm{X}$-shooter spectral coverage, down to $\sim 375 \mathrm{~nm}$. In the continuum are absorption features, which we identify as due to metal lines from $\mathrm{Zn}$ II, Fe II, and $\mathrm{Mg}$ II. Additionally, $\mathrm{Ly} \alpha$-absorption is seen at the end of the trace. The spectral position of the lines means that this GRB is at $z=2.087$. From the Ly $\alpha$ trough, we infer $\log \left(N_{\mathrm{HI}} / \mathrm{cm}^{-2}\right)=$ $21.7 \pm 0.4$. The afterglow continuum appears depressed in the blue end of the spectrum, suggestion dust extinction in the host.

\section{C.90. GRB 150301B $(z=1.517)$}

This spectrum has already been used in GCN 17523 (de Ugarte Postigo et al. 2015a), but it is not used elsewhere. Starting $5.1 \mathrm{~h}$ after the BAT trigger, this spectrum is based on $6 \times 600 \mathrm{~s}$ spectroscopic integration. The GRB afterglow continuum is well detected across the entire spectral coverage of X-shooter at moderate $\mathrm{S} / \mathrm{N}$. Imprinted on the continuum are absorption features which we identify as being caused by $\mathrm{Si}$ II, C IV, $\mathrm{Al}$ II, Fe II, Mg II, and $\mathrm{Mg}$ I- all at a similar redshift of $z=1.517$, which is likely the redshift of the GRB.

\section{C.91. GRB 150403A $(z=2.057)$}

This spectrum has previously been used to form the basis for GCN 17672 (Pugliese et al. 2015). $4 \times 600$ s spectroscopic integration, starting $10 \mathrm{~h}$ after the GRB trigger, captures a bright GRB afterglow. A broad absorption trough centred at $\sim 370 \mathrm{~nm}$ due to Ly $\alpha$ signals the redshift of the GRB, which is refined to $z=2.057$ based on the additional detection of metal absorption lines. We readily identify features associated with $S_{\text {II, }}$ Si Iv, O I, Si II, Si II ${ }^{*}, \mathrm{C}_{\text {II }}, \mathrm{C}_{\text {II }}{ }^{*}, \mathrm{C}$ IV, $\mathrm{Al}$ II, Fe II, Fe II ${ }^{*}, \mathrm{Mn}$ I, Mg II, and $\mathrm{Mg}_{\mathrm{I}}$ in the host of the GRB. An intervening $\mathrm{C}_{\mathrm{IV}}$ absorber is additionally detected at $z=1.76$. From the absorption trough due to $\operatorname{Ly} \alpha$, we infer the amount of neutral hydrogen in the host along the line of sight to be $\log \left(N_{\mathrm{HI}} / \mathrm{cm}^{-2}\right)=21.8 \pm 0.2$.

\section{C.92. GRB 150423A $(z=1.394)$}

The gross content of these observations have previously been presented in GCN 17755 (Malesani et al. 2015a), but is not published as part of any refereed paper. This bona-fide short GRB (T90 = is $0.22 \pm 0.03 \mathrm{~s}$; Lien et al. 2016) was observed in RRM mode and spectroscopic integration started after only $22 \mathrm{~min}$. A series of stare mode observations, increasing in exposure time, and ending with a nodding sequence, totalling $\sim 5000 \mathrm{~s}$, are combined to form this spectrum. A faint, almost featureless continuum is detected at low $\mathrm{S} / \mathrm{N}$ all the way to the bluest part of the spectrum. An absorption doublet is detected in the VIS arm against the GRB afterglow continuum, which we identify as $\mathrm{Mg}$ II at $z=1.394$.

\section{C.93. GRB 150428A $(z=n a)$}

This spectrum is empty, but is included here for completeness. It is not published anywhere. $4 \times 600 \mathrm{~s}$ spectroscopic observation, starting $3.7 \mathrm{~h}$ after the trigger does not reveal anything conclusive. The host association is additionally ambiguous for this, likely reddened (GCN 17767; Knust et al. 2015), GRB.

\section{C.94. GRB 150514A $(z=0.807)$}

This spectrum has already been used in GCN 17822 (de Ugarte Postigo et al. 2015b). Spectroscopic observations began $28.4 \mathrm{~h}$ post trigger and consist of a $4 \times 600 \mathrm{~s}$ nodding sequence. The GRB afterglow continuum is detected at moderate $\mathrm{S} / \mathrm{N}$ across the entire spectral coverage of $\mathrm{X}$-shooter. Narrow absorption features are imprinted in the continuum where we identify features from multiple Fe II transitions as well as the $\mathrm{Mg}$ II-doublet and the $\mathrm{Mg}_{\mathrm{I}} \lambda 2852.96$ resonance line. These lines are all found at a position matching $z=0.807$, suggesting it is the redshift of the GRB.

\section{C.95. GRB 150518A $(z=0.256)$}

This gross content of this data has previously been issued in GCN 17832 (Xu et al. 2015a), but is not published. Starting $>1$ day after the GRB trigger, $4 \times 600 \mathrm{~s}$ spectroscopic integration securely allows us to measure the redshift of the host. A continuum is detected all the way through the spectral coverage with multiple emission lines superposed. It is not clear to what degree the GRB afterglow contributes to the continuum. We identify the emission lines as [O II], $\mathrm{H} \beta,\left[\mathrm{O}_{\mathrm{III}}\right], \mathrm{H} \alpha,\left[\mathrm{N}_{\mathrm{II}}\right], \mathrm{H} \alpha$, and [S II] - all at $z=0.256$. Due to the spatial proximity of this burst, it is a candidate for $\mathrm{SN}$ follow-up and indeed there are indications of re-brightening at the burst position (GCN 17903; Pozanenko et al. 2015).

\section{C.96. GRB 150616A $(z=1.188)$}

These host observations are taken long after the burst had faded and have not been published before. They are included here for completeness. This bursts is excluded from the sample because an observing constraint delayed the Swift slew, causing the XRT observations to begin $16 \mathrm{~min}$ post trigger. No continuum is detected, but emission lines from [O $\mathrm{II}],\left[\mathrm{O}_{\mathrm{III}}\right]$, and $\mathrm{H} \alpha$ are all detected at $z=1.188$, setting it as the redshift of the GRB host. 
C.97. GRB 150727A $(z=0.313)$

The overall content of these observations have previously been reported in GCN 18080 (Tanvir et al. 2015a), but is not published. Starting $5 \mathrm{~h}$ after the Swift GRB trigger, these observations consist of a combined $2 \times 1200 \mathrm{~s}$ and $2 \times 600 \mathrm{~s}$ integration. A blue continuum is detected across the entire spectral coverage of X-shooter, suggesting a significant contribution from the GRB afterglow. Superposed on the continuum are emission lines which we identify as $\mathrm{H} \beta$, [O III], and $\mathrm{H} \alpha$ with a measured redshift of $z=0.313$. Supporting this redshift is the tentative detection of the $\mathrm{Mg}$ II absorption doublet in the afterglow continuum. The relative strength of the lines suggest that the line-forming region is dust obscured, contrary to the story told by the blue afterglow continuum.

\section{C.98. GRB 150821A $(z=0.755)$}

The gross content of this spectacular spectrum has already been issued in GCN 18187 (D'Elia et al. 2015a), but the spectrum has not been published. Starting just $12.4 \mathrm{~min}$ after the onboard trigger of Swift, a total of $4 \times 600$ s spectroscopic integration was obtained. The observations began just before dawn and the last two exposures are heavily affected by the brightening sky. A bright, high S/N GRB afterglow continuum is detected all across the spectral window and imprinted on this are a myriad of absorption features. We identify individual lines from transitions in $\mathrm{Al}$ III, Cr II, $\mathrm{Zn}$ II, Ni II ${ }^{*}, \mathrm{Fe}$ II, Fe II*, Sc II, Mn II, Mg II, Mg I, Ti II, and Ca II - all at a consistent redshift of $z=0.755$. The detection of fine-structure lines, excited local to the burst, clearly marks this as the redshift of the GRB.

\section{C.99. GRB 150910A $(z=1.359)$}

This spectrum has not previously been published. Starting $\sim 20 \mathrm{~h}$ after the BAT trigger, these observations were stopped after $2 \times 600 \mathrm{~s}$, when it became apparent that only a modest $\mathrm{S} / \mathrm{N}$ was obtainable, and the redshift had already been published in GCN 18273 (Zheng et al. 2015) and GCN 18274 (de Ugarte Postigo et al. 2015c). The continuum is detected at low $\mathrm{S} / \mathrm{N}$ all across $\mathrm{X}$-shooter and $\mathrm{Mg}$ II is detected at the suggested redshift, which we take as the redshift of the GRB.

\section{C.100. GRB 150915A $(z=1.968)$}

The gross content of these spectra have already been issued in GCN 18318 (D'Elia et al. 2015b). Observations began $3.3 \mathrm{~h}$ after the Swift trigger and lasted for $4 \times 1200 \mathrm{~s}$. A moderate $\mathrm{S} / \mathrm{N}$ afterglow continuum is detected across the entire spectral coverage of X-shooter, where the bluest part is affected by Ly $\alpha$-forest absorption. Imprinted on the afterglow continuum are a wealth of both emission and absorption features, all caused in a system for which we measure a redshift of $z=1.968$. We identify in emission $\left[\mathrm{O}_{\mathrm{II}}\right], \mathrm{H} \beta,\left[\mathrm{O}_{\mathrm{III}}\right]$, and $\mathrm{H} \alpha$ and absorption due to $\mathrm{Ly} \alpha, \mathrm{C}_{\mathrm{IV}}$, $\mathrm{Al}$ II, Si II, Fe II, and Mg II. We additionally identify fine-structure absorption lines from $\mathrm{Si} \mathrm{II}^{*}$ and $\mathrm{Fe} \mathrm{II}^{*}$, at a similar redshift, which unequivocally sets the suggested redshift as the redshift of the GRB. The Ly $\alpha$-line is affected by an atmospheric transmission drop and Ly $\alpha$ emission in the trough, making the measurement of the neutral hydrogen column density difficult. We measure $\log \left(N_{\mathrm{HI}} / \mathrm{cm}^{-2}\right) 21.2 \pm 0.3$.

\section{C.101. GRB 151021A $(z=2.330)$}

The data presented here also formed the basis of GCN 18426 (Pugliese et al. 2016) and are not published elsewhere. The observation was carried out in RRM starting 44 min after the GRB trigger. We detect absorption features from a wide range of ions at $z=2.330$ as well as intervening absorption at $z=1.49$.

\section{C.102. GRB 151027B $(z=4.063)$}

The content of these spectra has previously been issued in GCN 18506 (Xu et al. 2015b), but is not published. Beginning $5 \mathrm{~h}$ after the BAT trigger, 4 spectroscopic integrations obtained in a nodding sequence, each lasting for $600 \mathrm{~s}$, securely allows us to measure a redshift for this GRB. Beginning at $\sim 470 \mathrm{~nm}$, the Ly $\alpha$-forest is clearly detected at high $\mathrm{S} / \mathrm{N}$ leading up to the broad Ly $\alpha$ absorption trough and the onset of a bright GRB afterglow continuum. Imprinted in the afterglow continuum are a range of absorption lines which we identify as due to $\mathrm{Si}$ II, Si II*, O I, $\mathrm{C}_{\text {II, }} \mathrm{C}_{\text {II }}{ }, \mathrm{C}_{\text {IV }}, \mathrm{Al}$ II, $\mathrm{Al}$ II, Fe II, and $\mathrm{Fe}_{\text {II }}{ }^{*}$. These lines are all detected at $z=4.063$ and the presence of fine-structure lines at a consistent redshift securely sets this as the redshift of the GRB. GRB 151027B was the 1000th GRB detected by Swift. The afterglow of this burst is the subject of a forthcoming publication (Greiner et al. 2018).

\section{C.103. GRB 151029A $(z=1.423)$}

The gross content of these observations have previously been described in GCN 18524 (Tanvir et al. 2015b). Starting only $1 \mathrm{~h}$ after the BAT trigger, $2 \times 600 \mathrm{~s}$ observations, extending into the morning twilight, were obtained of this GRB. The brightening sky affects the $\mathrm{S} / \mathrm{N}$ of these spectra - especially in the blue end. The GRB afterglow is well detected over the entire spectral coverage of X-shooter, with spectral features from Fe II and $\mathrm{Mg}$ II imprinted by an absorber at $z=1.423$. The absorber is likely the host of the GRB and we therefore consider $z=1.423$ the redshift of the GRB.

\section{C.104. GRB 151031A $(z=1.167)$}

The spectral features in this spectrum has previously been reported in GCN 18540 (Malesani et al. 2015b). Observed in RRM mode, these observations were initiated only 19 min after the BAT trigger. As per the standard RRM scheme, a series of stare observation, increasing in en exposure time and ending with a regular $4 \times 600 \mathrm{~s}$ nodding sequence, were acquired for this burst. A moderate $\mathrm{S} / \mathrm{N}$ continuum is detected all the way through the X-shooter spectral coverage. In the GRB afterglow continuum, we identify absorption features as Fe II, Mg II, Mg I, and $\mathrm{Ca}$ II at a consistent redshift of $z=1.167$. Supporting this as the redshift of the GRB host galaxy are the simultaneous detection of nebular emission lines from [O $\mathrm{II}], \mathrm{H} \beta$, and [O III].

\section{C.105. GRB 160117B $(z=0.870)$}

A preliminary analysis of these spectra have previously been presented in GCN 18886 (de Ugarte Postigo et al. 2016a). Starting $13.5 \mathrm{~h}$ after the Swift trigger, these spectra are formed on the basis of $4 \times 1200 \mathrm{~s}$ spectroscopic integration. The GRB afterglow is clearly detected at high $\mathrm{S} / \mathrm{N}$ across the entire spectral coverage of X-shooter. Superposed on the continuum are absorption and emission features, which are identified as Fe II, $\mathrm{Mg}_{\text {II, }} \mathrm{Mg}_{\mathrm{I}}$ in 
absorption, and [O $\left.\mathrm{O}_{\mathrm{II}}\right], \mathrm{H} \beta$, and [O $\left.\mathrm{III}\right]$. The spectral positions of these lines all correspond to a redshift of $z=0.870$, which most likely is the redshift of the GRB.

\section{C.106. GRB 160203A $(z=3.518)$}

The data presented here also formed the basis of GCN 18982 (Pugliese et al. 2016) and are not published elsewhere. The observation was carried out in RRM starting $18 \mathrm{~min}$ after the GRB trigger. We detect absorption features from a wide range of ions at $z=3.518$ as well as intervening absorption at $z=$ 2.203 . From the very high $\mathrm{S} / \mathrm{N}$ spectrum we are able to measure $\log \left(N_{\mathrm{HI}} / \mathrm{cm}^{-2}\right)=21.75 \pm 0.10$, based on the shape of the Ly $\alpha$ profile. This burst is the subject of a forthcoming publication (Pugliese et al., in prep.).

\section{C.107. GRB 160228A $(z=1.640)$}

The gross content of these spectra have previously been presented in GCN 19186 (Krühler et al. 2016a). Taken long after the burst had faded, the target is the likely host galaxy. In $4 \times 1200 \mathrm{~s}$ spectroscopic integration we detected emission lines from the host. We find [O III] and $\mathrm{H} \alpha$ at a consistent $z=1.640$. We therefore suggest that this is the redshift of the GRB.

\section{C.108. GRB 160303A $(z=n a)$}

This is potentially a short burst with extended emission (GCN 19148; Ukwatta et al. 2016). The content of the spectra have previously been reported in GCN 19154 (de Ugarte Postigo et al. 2016b). Observations began $19.1 \mathrm{~h}$ after the BAT trigger and consist of $4 \times 1200 \mathrm{~s}$ spectroscopic integration. A very faint continuum is detected in the UVB and VIS arm after binning in the dispersion direction, but no clear redshift can be inferred from the continuum shape. No emission lines are readily apparent, suggesting little or no star formation in the host.

\section{C.109. GRB 160314A $(z=0.726)$}

The gross content of these spectra have previously been issued in GCN 19192 (D'Elia et al. 2016). Spectroscopic integration began $13 \mathrm{~h}$ post GRB trigger and lasted for $4 \times 1200 \mathrm{~s}$. In the data we see a moderate $\mathrm{S} / \mathrm{N}$ continuum across the entire spectral coverage of $\mathrm{X}$-shooter. Clearly visible on top of the continuum are emission lines from [O II], $\mathrm{H} \delta, \mathrm{H} \gamma, \mathrm{H} \beta,[\mathrm{O}$ III], $\mathrm{H} \alpha$, [N II] , and [S II] at a consistent redshift of $z=0.726$. It is unclear how much of the continuum is due to the host and how much is from the GRB afterglow. At the same redshift, we see the tentative detection of Fe II-absorption, supporting the redshift measurement and suggesting a significant afterglow contribution to the continuum.

\section{C.110. GRB 160410A $(z=1.717)$}

This short GRB with extended emission (GCN 19276; Sakamoto et al. 2016) was observed in RRM mode, with observations starting after only $7.7 \mathrm{~min}$. The gross content has already been presented in GCN 19274 (Selsing et al. 2016a). These observations have the shortest delay between trigger time and start of observation. Spectroscopic integration began after $8.4 \mathrm{~min}$ and lasted for $3 \times 600 \mathrm{~s}$. The last image in the nodding sequence was aborted because the object was setting and the telescope reached the hardware limit. A blue, moderate $\mathrm{S} / \mathrm{N}$ continuum is detected across the entire spectral coverage of X-shooter with multiple absorption features imprinted on it. At $z=1.717$ we detected the broad absorption trough due to $\operatorname{Ly} \alpha$ and we additionally find $\mathrm{Al}$ II and $\mathrm{Fe}$ II at a consistent redshift, setting it as the redshift of the GRB. Additionally, two intervening $\mathrm{C}$ IV absorption systems are detected at $z=1.444$ and $z=1.581$. From the Ly $\alpha$ absorption trough, we measure a neutral hydrogen column density of $\log \left(N_{\mathrm{HI}} / \mathrm{cm}^{-2}\right)=21.2 \pm 0.2$. This is the only short GRB for which we have a measurement of $N_{\mathrm{HI}}$ and is the subject of an forthcoming paper (Selsing et al., in prep.).

\section{C.111. GRB 160425A $(z=0.555)$}

These spectra were already used to form the basis for GCN 19350 (Tanvir et al. 2016a), but are not published anywhere else. Spectroscopic integration started after $7.2 \mathrm{~h}$ and lasted for $4 \times 1200 \mathrm{~s}$. A low S/N continuum is detected across the spectral coverage of $\mathrm{X}$-shooter, but no clear absorption lines are discernible. It is therefore unclear how much of the continuum is due to the host and how much is due to the GRB. Superposed on the continuum are two sets of emission lines, offset spatially, but at very similar redshifts. We identify lines from [O II], $\mathrm{H} \beta$, [O III], $\mathrm{H} \alpha,\left[\mathrm{N}_{\mathrm{II}}\right]$, and $\left[\mathrm{S}_{\mathrm{II}}\right]$ in both systems - all at $z=0.555$. As per GCN 19350 (Tanvir et al. 2016a), the GRB probably occurred in an interacting pair of galaxies.

\section{C.112. GRB 160625B $(z=1.406)$}

GRB $160625 B$ is the first GRB for which a significant linear polarization was measured (Troja et al. 2017a). Because this burst was detected by Fermi, it is therefore not a part of the statistical sample. The gross content of these spectra has previously been presented in GCN 19600 (Xu et al. 2016a). Starting 30 h after the trigger, these spectra are obtained in $4 \times 600 \mathrm{~s}$. The GRB afterglow continuum is detected at high $\mathrm{S} / \mathrm{N}$ throughout the spectral coverage of X-shooter. Imprinted on the afterglow continuum are a multitude of absorption features, from which we identify the responsible elements as $\mathrm{Si}$ II, O I, Si IV, C IV, Al II, Al III, Fe II, Zn II, Mg II, and Mg I at a consistent redshift of $z=1.406$. An additional intervening $\mathrm{Mg}$ II absorber is detected at $z=1.319$.

\section{C.113. GRB 160804A $(z=0.736)$}

The data presented here also formed the basis of GCN 19773 (Xu et al. 2016b), and are published in Heintz et al. (2018b). Observations started $22.37 \mathrm{~h}$ after the BAT trigger and lasted for $2.4 \mathrm{ks}$. The afterglow continuum is detected across the entire spectral coverage of $\mathrm{X}$-shooter and absorption lines from $\mathrm{Mg}$, $\mathrm{Mg}$ II, Fe II and $\mathrm{Al}$ II are found at $z=0.736$. At the same redshift, emission lines from [O ${ }_{\text {II }}$, [O III], $\mathrm{H} \alpha, \mathrm{H} \beta, \mathrm{H} \gamma,\left[\mathrm{N}_{\mathrm{II}}\right], \mathrm{S}_{\mathrm{II}}$, and [S III] are found. A second epoch, lasting $3.6 \mathrm{ks}$, is obtained after the afterglow had faded, confirming the emission line detections. The host galaxy is found to have a roughly solar metallicity and is among the most luminous GRB hosts at $z<1$.

\section{C.114. GRB 161001A $(z=0.891)$}

The content and scope of these spectra has previously been issued in GCN 19971 (Krühler et al. 2016b). 4 ×600 s spectroscopic integration was obtained of this potentially short GRB (GCN 19974; Markwardt et al. 2016), starting 6.1 h after the GRB trigger. Close to the XRT error circle (Osborne et al. 2016) there is a bright, point-like source, also visible in archival images. Our spectrum reveals that this is an M-type star, based 
on the presence of $\mathrm{TiO}$ bands, and is thus unrelated to the GRB. Two more objects lie within or in the near proximity of the X-ray error circle, as visible in our images from the acquisition camera. One is blended with the above mentioned star, and is serendipitously covered by the $\mathrm{X}$-shooter slit, revealing emission lines from [O $\left.\mathrm{O}_{\mathrm{II}}\right], \mathrm{H} \beta$, and $\mathrm{H} \alpha$ at a consistent redshift of $z=0.891$. The second source, first noted by Chen et al. (2016), is extended and lies just outside the XRT error circle. Based on GROND imaging, Chen et al. (2016) report moderate variability, and suggest this object as an afterglow candidate. Following our original imaging, we thus secured further late-time photometry with the X-shooter acquisition camera, starting on 2016 Oct 5.2 UT (4.22 days after the GRB), securing $3 \times 120 \mathrm{~s}$ in each of the $g$ and $r$ filters. No variability is apparent in either bands. In offline analysis of the GROND data (T. Krühler, priv. comm.), the evidence for fading is less significant than originally reported. The situation is thus left ambiguous, but due to the consistency of the $z=0.891$ object with the XRT localization, we consider this as the most likely redshift of GRB 161001A.

\section{C.115. GRB 161007A $(z=n a)$}

These data have not been published elsewhere. Observations for GRB 161007A started $323 \mathrm{~h}$ after the burst trigger and targeted the potential host galaxy (see GCN 20014, de Ugarte Postigo et al. 2016c and GCN 20020, Heintz et al. 2016). $4 \times 600 \mathrm{~s}$ of observations were obtained, revealing a faint continuum rising abruptly above the noise at $\sim 685 \mathrm{~nm}$ and extending up to $2100 \mathrm{~nm}$. A very low significance continuum is detected at shorter wavelengths, down to $\sim 600 \mathrm{~nm}$. No significant emission features could be identified. Two possibilities can explain the observed continuum break, but neither is fully satisfactory. The first option is the $\operatorname{Ly} \alpha$ cutoff at $z \sim 4.6$; at this redshift, the usually detected strong nebular lines would be shifted out of the covered wavelength range. The host would be however exceptionally bright, with an absolute magnitude at $\approx 150 \mathrm{~nm} M=-23.8 \mathrm{AB}$, that is about 2.8 mag brighter than $M^{*}$ at that redshift (e.g. Bouwens et al. 2015). In order to test this option, we secured $B$-band imaging (using the ESO/VLT UT1 equipped with the FORS2 camera) of the host, yielding a faint but significant detection $(B=25.48 \pm 0.13 \mathrm{Vega})$. As the $B$ band would lie entirely bluewards of the Lyman limit at $z=4.6$, where negligible flux is expected to be transmitted by the IGM, our detection rules out this solution. A second solution is that the continuum discontinuity is the $400 \mathrm{~nm}$ break at $z=0.71$, such feature is however more prominent in evolved galaxies, which are unlikely hosts of long-duration GRBs (but see Rossi et al. 2014). A fit to the sparse available photometry, including our FORS2 datum, the GTC $i$-band value (de Ugarte Postigo et al. 2016c), Pan-STARRS measurements (Chambers et al. 2016), and WISE (Wright et al. 2010) W1 detection $(W 1=19.72 \pm 0.15)$, is unable to tightly constrain the redshift $(0.47<z<1.34)$. Fixing $z=0.7$ yields however a significant star formation rate $\left(25-200 M_{\odot} \mathrm{yr}^{-1}\right)$, which is in contrast with the lack of detected emission lines in the spectrum (the expected $\mathrm{H} \alpha$ flux would be $10^{-15}-10^{-16} \mathrm{erg} \mathrm{cm}^{-2} \mathrm{~s}^{-1}$, easily detectable). As such we cannot identify a self-consistent solution. Given the lack of optical variability of the candidate counterpart, it cannot be excluded that the object within the XRT error circle is a chance association, as the probability of a random alignment is small but non negligible (of the order of $1 \%$ ). In conclusion, we cannot provide a secure redshift for GRB 161007A.

\section{C.116. GRB 161014A $(z=2.823)$}

The data presented here also formed the basis of GCN 20061 (Selsing et al. 2016b), but are not published elsewhere. Starting $11.6 \mathrm{~h}$ after the GRB trigger, $4.8 \mathrm{ks}$ of integration time captures the afterglow continuum across all three spectroscopic arms. A broad absorption trough due to Lyman alpha is visible, along with metal absorption features from $\mathrm{Mg}_{\text {II, }} \mathrm{Si}$ II, $\mathrm{C}_{\text {II }}, \mathrm{C}_{\text {IV }}, \mathrm{Al}_{\text {II, }}$, Al III, and Fe II, all at $z=2.823$. Similar to GRB 140506 (Fynbo et al. 2014b; Heintz et al. 2018b), a break in the continuum shape is tentatively detected bluewards of $600 \mathrm{~nm}$, possible signifying some anomalous form of extinction.

\section{C.117. GRB 161023A $(z=2.710)$}

The gross content of these spectra have previously been reported in GCN 20104 (Tanvir et al. 2016b) and is published in de Ugarte Postigo et al. (2018). This extremely bright INTEGRAL burst is not a part of the statistical sample. Starting $3 \mathrm{~h}$ after the trigger, the spectra are based on $2 \times 600 \mathrm{~s}$. The GRB afterglow continuum is detected with the highest $\mathrm{S} / \mathrm{N}$ of all the spectra presented here. A myriad of absorption features are visible against the continuum, many of which would not be visible against lower $\mathrm{S} / \mathrm{N}$ spectra. We identify at least 15 intervening absorbers between $z=1.243$ and $z=2.710$. In the host system we clearly see absorption from $\operatorname{Ly} \beta$, Ly $\alpha, S_{\text {II, Si II, }}$ O I, Si Iv, C Iv, Al II, Al III, Fe II, Mg II, and Mg I. Several finestructure transitions are additionally seen, clearly associating the $z=2.710$ system with the GRB. From the Ly $\alpha$-line we infer $\log \left(N_{\mathrm{HI}} / \mathrm{cm}^{-2}\right)=20.96 \pm 0.05$.

\section{C.118. GRB 161117A $(z=1.549)$}

The majority of the spectral features of these spectra have previously been reported in GCN 20180 (Malesani et al. 2016). Starting only $\sim 40 \mathrm{~min}$ after the BAT trigger, these observations consist of $4 \times 600 \mathrm{~s}$, taken under relatively poor observing conditions. The GRB afterglow continuum is detected throughout most of the X-shooter spectral coverage at low-to-moderate $\mathrm{S} / \mathrm{N}$, however the continuum level drops below the noise in the bluest part of the UVB arm at $\sim 380 \mathrm{~nm}$. Imprinted in the continuum are absorption features, which we identify as due to Fe II, Mg II, and $\mathrm{Mg}_{\mathrm{I}}$ in an absorption system at $z=1.549$. We consider this the likely redshift of the GRB.

\section{C.119. GRB 161219B $(z=0.148)$}

The core signatures in these spectra have already been issued in GCN 20321 (Tanvir et al. 2016c), and are additionally used in both Ashall et al. (2017) and Cano et al. (2017). This GRB is very closeby and is associated with the SN Ic-BL SN2016jca (GCN 20342; de Ugarte Postigo et al. 2016d). The observations consist of $4 \times 600$ s spectroscopic integration, starting 1.5 days after the GRB trigger. The GRB afterglow continuum is detected at high $\mathrm{S} / \mathrm{N}$ across the entire spectral coverage of X-shooter. Superposed on the continuum are absorption features, which we identify as being formed by the $\mathrm{Mg}$ II, Mg I, and the $\mathrm{CaH} \& \mathrm{~K}$-lines at $z=0.148$. Underneath the glaring afterglow continuum we additionally detect nebular emission from [O $\mathrm{II}], \mathrm{H} \beta,\left[\mathrm{O}_{\mathrm{III}}\right], \mathrm{H} \alpha$, and [ $\left.\mathrm{S}_{\mathrm{II}}\right]$ at a consistent redshift, which is the likely redshift of the GRB.

\section{C.120. GRB 170113A $(z=1.968)$}

These gross content of these data have previously been issued in GCN 20458 (Xu et al. 2017). Starting 15.2 h after the BAT 
trigger, these observations consist of $4 \times 1200 \mathrm{~s}$ spectroscopic integration. The GRB afterglow continuum is detected at moderate $\mathrm{S} / \mathrm{N}$ across the entire spectral coverage of $\mathrm{X}$-shooter. In the bluest end of the spectral coverage, the background noise increases, thus lowering the continuum below detection. Superposed on the afterglow continuum are both absorption lines and emission lines. In absorption we identify lines from Si II, Fe II, and $\mathrm{Mg}$ II, and in emission we detect [O II], $\mathrm{H} \beta$, and [O III]. All the lines are detected at a consistent redshift of $z=1.968$, which we take as the redshift of the GRB.

C.121. GRB 170202A $(z=3.645)$

GRB 170202A is the last GRB afterglow observed in this work. It concludes the follow-up effort that has lasted for
8 years. The majority of the spectral features in this spectrum have previously been reported in GCN 20589 (Palmerio et al. 2017). Spectroscopy began $9.7 \mathrm{~h}$ after the BAT trigger, and lasted for $4 \times 600 \mathrm{~s}$. The GRB afterglow is well detected at moderate-to-high $\mathrm{S} / \mathrm{N}$ throughout the spectral coverage of $\mathrm{X}$ shooter, down to $430 \mathrm{~nm}$. The lowest wavelength continuum from $430 \mathrm{~nm}$ to $560 \mathrm{~nm}$ is absorbed by the Ly $\alpha$ forest, with the broad Ly $\alpha$ absorption trough centred at $565 \mathrm{~nm}$. In the continuum, we additionally identify metal absorption features from $\mathrm{Si}$ II, C IV, Si Iv, Fe II, and Mg II, with the additional detection of $\mathrm{Si}_{\text {II }}^{*}$ fine-structure lines. With an additional detection of [O III] and all lines detected at $z=3.645$, this unequivocally marks this as the redshift of the GRB. There is an additional $\mathrm{C}$ IV absorber at $z=3.077$. From the Ly $\alpha$ absorption we measure $\log \left(N_{\mathrm{HI}} / \mathrm{cm}^{-2}\right)=21.55 \pm 0.10$. 